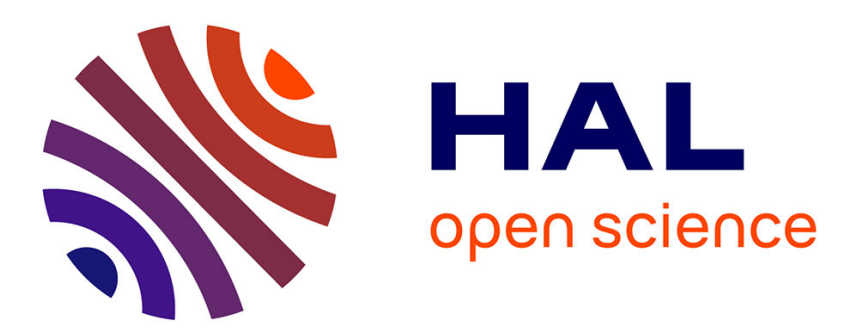

\title{
Homogenization of a Multiscale Viscoelastic Model with Nonlocal Damping, Application to the Human Lungs
}

\author{
Paul Cazeaux, Céline Grandmont
}

\section{To cite this version:}

Paul Cazeaux, Céline Grandmont. Homogenization of a Multiscale Viscoelastic Model with Nonlocal Damping, Application to the Human Lungs. Mathematical Models and Methods in Applied Sciences, 2015, 25 (6), pp.1125. 10.1142/S0218202515500293 . hal-00873549

HAL Id: hal-00873549

https://hal.inria.fr/hal-00873549

Submitted on 15 Oct 2013

HAL is a multi-disciplinary open access archive for the deposit and dissemination of scientific research documents, whether they are published or not. The documents may come from teaching and research institutions in France or abroad, or from public or private research centers.
L'archive ouverte pluridisciplinaire HAL, est destinée au dépôt et à la diffusion de documents scientifiques de niveau recherche, publiés ou non, émanant des établissements d'enseignement et de recherche français ou étrangers, des laboratoires publics ou privés.

\section{(c)(1)}

Distributed under a Creative Commons Attribution| 4.0 International License 


\title{
Homogenization of a Multiscale Viscoelastic Model with Nonlocal Damping, Application to the Human Lungs*
}

\author{
PAUL CAZEAUX, \\ Laboratoire J.-L. Lions, UMR 7598, Université Pierre et Marie Curie-Paris 6, \\ 4 Place Jussieu, Paris, F-75005, France \\ E Inria Projet REO, Rocquencourt, BP 105, F-78153 Le Chesnay Cedex, France \\ cazeaux@ann.jussieu.fr \\ CÉLINE GRANDMONT, \\ Laboratoire J.-L. Lions, UMR 7598, Université Pierre et Marie Curie-Paris 6, \\ 4 Place Jussieu, Paris, F-75005, France \\ \& Inria Projet REO, Rocquencourt, BP 105, F-78153 Le Chesnay Cedex, France \\ celine.grandmont@inria.fr
}

\begin{abstract}
We are interested in the mathematical modeling of the deformation of the human lung tissue, called the lung parenchyma, during the respiration process. The parenchyma is a foam-like elastic material containing millions of air-filled alveoli connected by a treeshaped network of airways. In this study, the parenchyma is governed by the linearized elasticity equations and the air movement in the tree by the Poiseuille law in each airway. The geometric arrangement of the alveoli is assumed to be periodic with a small period $\varepsilon>0$. We use the two-scale convergence theory to study the asymptotic behavior as $\varepsilon$ goes to zero. The effect of the network of airways is described by a nonlocal operator and we propose a simple geometrical setting for which we show that this operator converges as $\varepsilon$ goes to zero. We identify in the limit the equations modeling the homogenized behavior under an abstract convergence condition on this nonlocal operator. We derive some mechanical properties of the limit material by studying the homogenized equations: the limit model is nonlocal both in space and time if the parenchyma material is considered compressible, but only in space if it is incompressible. Finally, we propose a numerical method to solve the homogenized equations and we study numerically a few properties of the homogenized parenchyma model.
\end{abstract}

Keywords: Mathematical modeling; Periodic homogenization; Two-scale convergence method; Fluid--structure interaction.

\section{Introduction and Motivation}

Breathing involves the transport of air through the respiratory tract from its external entries, the nose and the mouth. During inspiration, the airflow moves down the pharynx and the trachea, where it is divided between left and right bronchi and enters the lungs. It is then distributed by the bronchial tree to the acini or alveolar

*This work was partially funded by the ANR-08-JCJC-013-01 (M3RS) project headed by C. Grandmont. 
sacs, embedded in a viscoelastic tissue, made in particular of blood capillaries and a network of elastic fibers. The alveoli are tiny bubble-like units where the gaseous exchanges occur. Thus, it is the function of the bronchial tree to efficiently supply them with fresh air via a tree-shaped structure. The coupled movement of air and tissue is achieved by displacement of the diaphragm and of the connective tissue framework of the lungs, which is usually called the parenchyma, [41]. Our motivation concerns the mathematical modeling of the human respiratory system and our interest here is to provide a simple model for the behavior of the alveolar region coupled with the bronchial tree during the respiration.

Modeling the mechanical behavior of the lungs, including both the air flow in the airway network and the 3D displacement of the parenchyma is difficult because of the complexity of the bronchial tree geometry and the porous, foam-like structure of the parenchyma. Moreover, for the time being it is far from possible to compute 3D Navier-Stokes airflow simulations on such a complex fractal geometry as that of the full airway tree, let alone a full fluid-structure interaction problem modeling the whole ventilation process on a realistic geometry of the lung.

It is thus necessary to develop models of reduced complexity, both to further the understanding of the lungs' mechanics and to be able to compute numerical simulations of the ventilation process. A possible choice is to describe the evolution of the air flux by a simple ODE model, as presented e.g. in [27]. Such models are certainly helpful for understanding the respiration mechanisms, but cannot yield precise information on the coupled 3D displacement of the parenchyma and airflow. Fully resolved flow computations are possible for the upper airways and the proximal part of the bronchial tree [7,15,23], but usually choose a set of ad hoc boundary conditions on the part of the tree which has been cut off. In [4], a coupled ventilation model was developed to include the parenchyma, represented by a simple spring model and connected to the ends of the upper part of the bronchial tree by a resistance. To couple such models of the bronchial tree with a three-dimensional representation of the parenchyma, the mechanics of the set of acini have to be represented by a reduced model. The purpose of the present work is to obtain rigorously such a model of reduced complexity for the alveolar region by using the tools of two-scale periodic homogenization, involving fluid-structure interaction in the porous domain and flow of air through the bronchial tree.

Other works related to multiscale modeling of the lungs' parenchyma include [33] and [36], where the alveoli structure is considered as a porous media and a formal homogenization approach is applied to a system coupling the linearized Navier-Stokes equations for air and linear viscoelasticity for the solid tissue. Other approaches have been proposed, such as discrete spring-mass systems [19] or equations empirically derived at the continuous level [22]. For the periodic homogenization of fluid-structure interaction systems, one may refer in particular to $[14,16,32,35]$, where the homogenization of the Stokes equations coupled to an elastic frame is performed. A homogenized model for the propagation of sound waves in cancellous bone is obtained in [13]. 
A mathematical description of the airflow in the bronchial tree as an abstract dyadic resistive tree was studied in depth in [39], and especially the asymptotic behavior as the number of generations of the tree grows to infinity. Moreover, this description has been used in [18] to obtain a one-dimensional model of the parenchyma by connecting a dyadic resistive tree with a system of springs and masses. The aim of this paper is then to extend this one-dimensional model to a multi-dimensional setting.

Our approach is based on a series of simplifying assumptions of the description of the lung mechanics. We assume that the alveoli are periodically arranged with a small period $\varepsilon>0$, as is suggested by the spatial arrangement of the acinus. To obtain a macroscopic description of the parenchyma, we are going to let the size of the microstructure, denoted by $\varepsilon$, go to zero and study the convergence of the displacement of the structure. Our aim is to obtain an homogenized model for the displacement of the parenchyma that takes into account the effect of the ventilation by the bronchial tree. As the number of alveoli grows to infinity, so does the number of generations of the airway tree that is feeding them with air. In order to describe the airflow through the airway tree we will assume that the Poiseuille law is satisfied in each airway. The bronchial tree can then be modeled as a branching network of airways, represented as a dyadic resistive tree (see e.g. [28, 29]). The airflow through the tree is then completely characterized by the knowledge of the individual resistances of the branches, which depends only on the dimensions of the bronchi and can be computed from available anatomical data [40]. Strictly speaking, the Poiseuille law is not valid for the first generations of the bronchial tree where one needs to take into account inertial effects. We will nevertheless assume here, for simplicity, that this description is valid for the whole airway tree.

We propose to model the alveoli as closed cavities in an elastic matrix, filled with air, as in the static parenchyma model proposed in [3]. Following [38], each alveolus is then connected to one end of our abstract dyadic resistive tree representing the bronchial tree. The air can flow in and out of the alveolus through this terminal branch of the tree. We assume also the parenchyma behaves like a linearized elastic material (i.e. the deformations are small). We propose to study the two cases of a compressible and an incompressible wall material. In both cases, we obtain boundary value problems (1.14) and (1.15) respectively in the compressible and incompressible case. Note that the well-posedness of this problem in the compressible case was studied in [38].

Once the geometric setting and the model are in place, we study the asymptotic behavior of the displacement fields as the microscale parameter $\varepsilon$ goes to zero and the number of generations of the tree goes to infinity. Unlike in the one-dimensional setting proposed in [18], there are many ways of connecting a tree to the periodically distributed alveoli in a three-dimensional domain. To deal with this difficulty, we define a Dirichlet-to-Neumann operator representing the action of the tree by relating the fluxes and the pressures in the alveoli, which can be seen as constant-by-cell functions in the domain $\Omega$ representing the parenchyma. This resistance operator 
is then a linear operator in $\mathcal{L}\left(L^{2}(\Omega)\right)$. We propose to use the strong convergence of the sequence of these resistance operators in the space $\mathcal{L}\left(L^{2}(\Omega)\right)$, as $\varepsilon$ goes to zero, as an abstract condition to model the convergent behavior of the sequence of trees ventilating our parenchyma domain. This allows us to divide the theoretical analysis in two parts.

In the first part, we build a connection between the sequence of trees and the alveoli such that, under some conditions on the geometry and the resistances of the tree, the abstract convergence condition holds true. In this paper, we present a practical example of such a construction, extending the one-dimensional analysis presented in [18]. A more generic analysis will be presented in a forthcoming paper, based on the construction proposed in [39].

In the second part, we present the main results of this paper as we show that this condition is sufficient to pass to the two-scale limit and obtain a homogenized model of the lung. This analysis is presented in Sections 3 and 4. First, we study the well-posedness for both problems and we show a priori bounds independent of $\varepsilon$. Then, using the two-scale homogenization method $[1,31]$ we analyze the asymptotic behavior of the displacement field solution of (1.14) and (1.15) as the micro-scale parameter $\varepsilon$ goes to zero. We focus in particular on the convergence of the non-standard terms describing the interaction of the tree and the structure. The main results of the paper are the convergence Theorems 3.2 and 4.2, which describe the macroscopic homogenized problems in the case of a compressible and an incompressible parenchyma structure, respectively.

We can analyze physically the mechanical behavior of the homogenized material we obtain in each case. When we suppose that the wall material is compressible, the homogenized parenchyma behaves like a compressible viscoelastic material with non-local damping both in space and time, thus showing some long-term memory effects. On the other hand, when the wall material is supposed to be incompressible, the homogenized material is compressible and viscoelastic with non-local damping in the space variable only. The non-local behavior is due to the effect of the abstract resistive tree which connects the different points of the domain. In this case, the loss of the long-term memory effect comes from the incompressibility of the constitutive elastic media.

Finally we conclude this work by a numerical study of our homogenized ventilation model, presented in Section 5. We present a finite elements numerical method designed to tackle the homogenized problems obtained in Sections 3 and 4. The main difficulty is the need to deal with the non-local operator associated with the tree as it writes as a full matrix in the finite elements basis. Our method is based on fast algorithms which take advantage of the tree structure to compute quickly the matrix-vector products associated with the viscous non-local operator. We present then some numerical simulations. The results show that we can make our parenchyma model breathe and that we can study the effects of the modification of some parameters, like the distal resistances or the stiffness of the material. 
The paper is organized as follows. In Section 1, we describe precisely the geometry and then write the equations of the coupled fluid-structure interaction models that we shall study. In Section 2, we introduce the resistance operators describing the action of the tree and an abstract convergence condition which we verify in a particular case. In Section 3, we derive the homogenized limit of the compressible model (1.14) by the method of two-scale convergence. In Section 4, we work out the same analysis for the incompressible model (1.15). Finally, in Section 5, we present a numerical method adapted to the homogenized model, and we present a few examples of numerical simulations with parameters inspired by realistic human lung's anatomical data.

Notations We use the common Kronecker symbol $\delta_{i j}=1$ if $i=j$ and 0 if $i \neq j$, and the Einstein summation convention with repeated indices. The symbol Id denotes the identity matrix, and given two tensors of order $2 A$ and $B$, we denote the contraction of tensors as

$$
A: B=a_{i j} b_{j i} .
$$

We will denote with bold characters vectors, vector-valued fields and functional spaces of vector-valued fields. Given any vector field $\mathbf{v}$, we denote $e(\mathbf{v})$ its symmetrized gradient

$$
e(\mathbf{v})=\frac{1}{2}\left(\nabla \mathbf{v}+(\nabla \mathbf{v})^{T}\right) .
$$

\section{Description of the model}

\subsection{Geometric setting}

The parenchyma model we propose to study is a porous media, homogeneously composed as a periodic arrangement of closed pores (modeling the alveoli), connected in an abstract way by a dyadic resistive tree modeling the pulmonary airways. Let us give a formal description of this material.

Let $d$ be the dimension, $d=2$, 3. First, we describe the geometrical setting of the alveoli by defining an open periodic cell $\mathcal{Y}$, normalized so that $|\mathcal{Y}|=1$. This unit cell is associated with a periodic array $\mathbf{Z}$ of $\mathbb{R}^{d}$, which is the discrete set of translation vectors such that $\mathcal{Y}+\mathbf{Z}$ is a tiling of the whole space.

Remark 1.1. The standard and most simple such unit cell is a square or a cube, associated with $\mathbf{Z}=\mathbb{Z}^{d}$. However other structures are more representative of the geometry of the alveoli, in particular the truncated octahedron is often used in the biology literature [37].

We further divide $\mathcal{Y}$ into two open sets: $\mathcal{Y}_{F}$ that represents an air cavity and $\mathcal{Y}_{S}$ that represents the elastic walls of the alveolus. Let $\theta=\left|\mathcal{Y}_{S}\right|$ be the volume fraction of the elastic material. More precisely, we suppose that $\mathcal{Y}_{F}$ is smooth, simply connected and that:

$$
\overline{\mathcal{Y}_{F}} \cup \overline{\mathcal{Y}_{S}}=\overline{\mathcal{Y}}, \quad \mathcal{Y}_{F} \cap \mathcal{Y}_{S}=\emptyset, \quad \overline{\mathcal{Y}_{F}} \subset \dot{\mathcal{Y}} .
$$




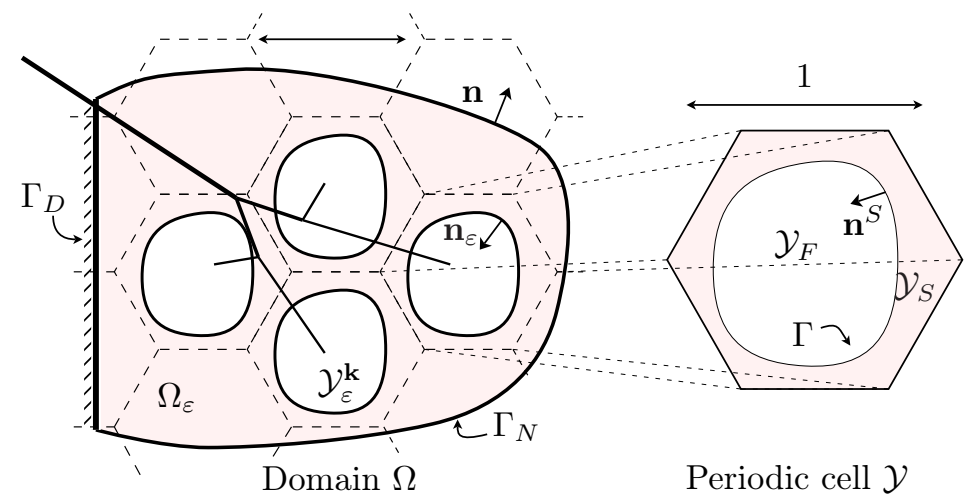

Figure 1: Parenchyma model

We also define $\Gamma=\partial \mathcal{Y}_{F}$ as the interior fluid-structure interface in the unit cell with unit normal $\mathbf{n}^{S}$ pointing into the fluid part.

Next, we model the space occupied by the lungs' parenchyma (or portion of the parenchyma) as $\Omega$, a nonempty bounded open domain in $\mathbb{R}^{d}$ with Lipschitz boundary $\partial \Omega$ and unit outward normal $\mathbf{n}$. An example is given in Figure 1. The boundary is decomposed as $\overline{\partial \Omega}=\overline{\Gamma_{D}} \cup \overline{\Gamma_{N}}$ where $\Gamma_{N} \cap \Gamma_{D}=\emptyset$ and $\left|\Gamma_{D}\right|>0$. We will suppose that the parenchyma is fixed at the portion of the boundary $\Gamma_{D}$.

Given a small parameter $\varepsilon>0$ and a multi-index $\mathbf{k} \in \mathbf{Z}$, we define

$$
\mathcal{Y}_{\varepsilon}^{\mathbf{k}}=\varepsilon(\mathcal{Y}+\mathbf{k}), \quad \mathcal{Y}_{F, \varepsilon}^{\mathbf{k}}=\varepsilon\left(\mathcal{Y}_{F}+\mathbf{k}\right), \quad \mathcal{Y}_{S, \varepsilon}^{\mathbf{k}}=\varepsilon\left(\mathcal{Y}_{S}+\mathbf{k}\right), \quad \Gamma_{\varepsilon}^{\mathbf{k}}=\varepsilon(\Gamma+\mathbf{k}),
$$

that is a translation and a homothetic transformation of the reference cells $\mathcal{Y}, \mathcal{Y}_{F}$, $\mathcal{Y}_{S}$ and of the fluid-structure interface $\Gamma$. Furthermore, by introducing the set of multi-indexes $\mathbf{Z}_{\varepsilon}^{\Omega}$ as:

$$
\mathbf{Z}_{\varepsilon}^{\Omega}=\left\{\mathbf{k} \in \mathbf{Z} \mid \mathcal{Y}^{\varepsilon, \mathbf{k}} \subset \Omega\right\}
$$

we define the periodically perforated structure domain and the interior interface as

$$
\Omega_{\varepsilon}=\Omega \backslash \bigcup_{\mathbf{k} \in \mathbf{Z}_{\varepsilon}^{\Omega}} \overline{\mathcal{Y}_{F, \varepsilon}^{\mathbf{k}}}, \quad \Gamma_{\varepsilon}=\bigcup_{\mathbf{k} \in \mathbf{Z}_{\varepsilon}^{\Omega}} \Gamma_{\varepsilon}^{\mathbf{k}} .
$$

We define a unit normal vector $\mathbf{n}_{\varepsilon}$ defined on the fluid-structure interface $\Gamma_{\varepsilon}$ and pointing to the exterior of the structure domain $\Omega_{\varepsilon}$. Let $\chi_{F}$ and $\chi_{S}$ be the characteristic functions of $\mathcal{Y}_{F}$ and $\mathcal{Y}_{S}$ respectively, $\chi_{F, \varepsilon}, \chi_{S, \varepsilon}$ the characteristic functions of $\Omega \backslash \Omega_{\varepsilon}=\bigcup_{\mathbf{k} \in \mathbf{Z}_{\varepsilon}^{\Omega}} \overline{\mathcal{Y}_{F, \varepsilon}^{\mathbf{k}}}$ and $\Omega_{\varepsilon}$, respectively, and $\chi_{\varepsilon}^{\mathbf{k}}$ the characteristic function of $\mathcal{Y}_{\varepsilon}^{\mathbf{k}}$.

Finally, we connect the alveoli $\mathcal{Y}_{F, \varepsilon}^{\mathbf{k}}$ filled with air to an abstract finite dyadic tree $\mathcal{T}_{\varepsilon}$ representing the geometrical connections of the bifurcating airway tree (see Figure 2). The tree $\mathcal{T}_{\varepsilon}$ is a subtree of the larger, infinite dyadic tree $\mathcal{T}$ characterized 
by the set of nodes

$$
V=X_{0} \cup\left\{X_{n, k}: n \in \mathbb{N}, 0 \leq k \leq 2^{n}-1\right\} .
$$

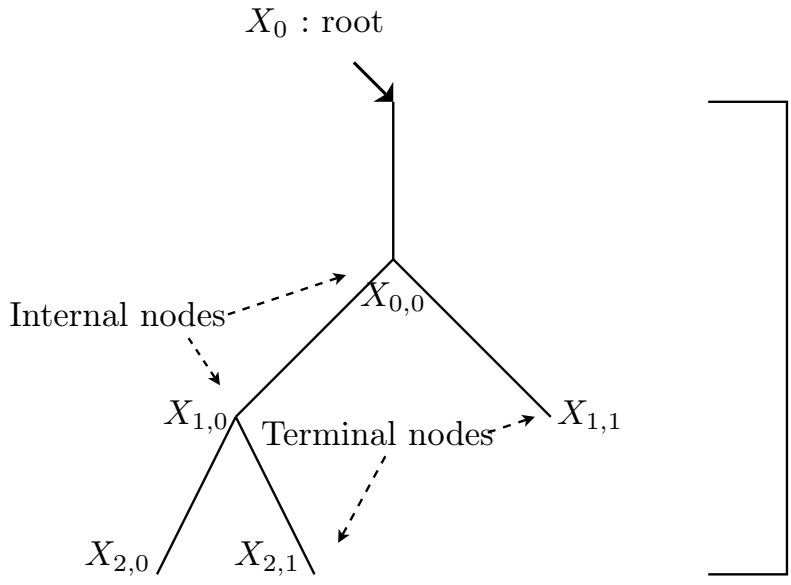

Tree $\mathcal{T}_{\varepsilon}$

$$
\text { Outlets: }(2,0) \quad(2,1) \quad(1,1)
$$

Figure 2: Finite dyadic tree

The tree $\mathcal{T}_{\varepsilon}$ is supposed to be full, meaning that if $X_{n, k} \in V$ is a node of $\mathcal{T}_{\varepsilon}$, then either both children of $X_{n, k}$ belong to $\mathcal{T}_{\varepsilon}\left(X_{n, k}\right.$ is then called an internal node of $\mathcal{T}_{\varepsilon}$ ), or neither does and $X_{n, k}$ is a terminal node (or a leaf) of $\mathcal{T}_{\varepsilon}$. We define the set of indexes $\mathcal{I}_{\varepsilon}$ and $\mathcal{E}_{\varepsilon} \subset \mathbb{N}^{2}$, indexing respectively the internal and terminal nodes of $\mathcal{T}_{\varepsilon}$ :

$$
\begin{aligned}
& \mathcal{I}_{\varepsilon}=\left\{(n, k) \in \mathbb{N}^{2}, X_{n, k} \text { is an internal node of the tree } \mathcal{T}_{\varepsilon}\right\}, \\
& \mathcal{E}_{\varepsilon}=\left\{(n, k) \in \mathbb{N}^{2}, X_{n, k} \text { is a leaf of the tree } \mathcal{T}_{\varepsilon}\right\},
\end{aligned}
$$

and we suppose that $\mathcal{E}_{\varepsilon}$ and $\mathbf{Z}_{\varepsilon}^{\Omega}$ have the same number of elements. Let $\omega_{\varepsilon}$ be a one-to-one mapping

$$
\omega_{\varepsilon}: \begin{cases}\mathbf{Z}_{\varepsilon}^{\Omega} & \rightarrow \mathcal{E}_{\varepsilon}, \\ \mathbf{k} & \mapsto(n, k) .\end{cases}
$$

The mapping $\omega_{\varepsilon}$ describes the connection between the tree and the alveoli. This lets us enumerate the cells of the periodically perforated domain $\Omega_{\varepsilon}$ following either the tree indexing by $\mathcal{E}_{\varepsilon}$, or the spatial indexing by $\mathbf{Z}_{\varepsilon}^{\Omega}$, and as long as there is no possible confusion we will use either set of indexes indifferently, for example:

$$
\mathcal{Y}_{\varepsilon}^{n, k}=\mathcal{Y}_{\varepsilon}^{\mathbf{k}} \quad \text { where }(n, k)=\omega_{\varepsilon}(\mathbf{k}) \in \mathcal{E}_{\varepsilon} \text { for } \mathbf{k} \in \mathbf{Z}_{\varepsilon}^{\Omega} .
$$


Remark 1.2. A more precise description of the relation between the geometric arrangement of the alveoli and their positions at the ends of the tree is given later in Section 1.1 with an example. We refer to a forthcoming paper for a complete description in a generic setting.

The key goal for obtaining a two-scale continuum is to derive a macroscopic description of the material from the description of the microstructure, here the alveolar set. The assumption of a small parameter, $\varepsilon>0$, implies that the fields depends on two spatial variables which belong to well-separated scales: the ordinary position vector $\mathbf{x} \in \Omega$, and the position vector in a stretched coordinate system $\mathbf{y}=\varepsilon^{-1} \mathbf{x}$. The variable $\mathbf{x}$ will be called slow and the variable $\mathbf{y}$ fast. By performing the asymptotic analysis when $\varepsilon$ goes to zero, we expect the two sets of variables to become independent.

\subsection{Description of the parenchyma model}

We now turn to the description of the mechanical behavior of the parenchyma. Here $\varepsilon$ is fixed, and we suppose that $\Omega_{\varepsilon}$ is a reference configuration for the elastic media. We shall analyze two cases: first, we will consider a linear homogeneous elastic material and then we will add to this model an incompressibility constraint.

We denote by $\mathbf{u}_{\varepsilon}$ the displacement field of the elastic media filling the perforated domain $\Omega_{\varepsilon}$. We are interested in small displacements around the reference configuration and we suppose that the material obeys the laws of linearized elasticity, see [8]:

$$
\left\{\begin{array}{rlrl}
\rho \partial_{t t} \mathbf{u}_{\varepsilon}-\operatorname{div} \sigma & =\mathbf{f}, & \text { in } \Omega_{\varepsilon}, \\
\sigma \mathbf{n}_{\varepsilon} & =-p_{\varepsilon}^{\mathbf{k}} \mathbf{n}_{\varepsilon}, & \text { on } \Gamma_{\varepsilon}^{\mathbf{k}}, \forall \mathbf{k} \in \mathbf{Z}_{\varepsilon}^{\Omega}, \\
\sigma \mathbf{n} & =-p_{N} \mathbf{n}, & & \text { on } \Gamma_{N}, \\
\mathbf{u}_{\varepsilon} & =\mathbf{0}, & & \text { on } \Gamma_{D},
\end{array}\right.
$$

Here, in the compressible case, $\sigma$ stands for the stress tensor associated with the displacement $\mathbf{u}_{\varepsilon}$, i.e.

$$
\sigma\left(\mathbf{u}_{\varepsilon}\right)=\lambda \operatorname{div}\left(\mathbf{u}_{\varepsilon}\right) \operatorname{Id}+2 \mu e\left(\mathbf{u}_{\varepsilon}\right)(\text { Hooke's law })
$$

where the parameters $\lambda>0$ and $\mu>0$ are the Lamé constants, constitutive of the elastic media, and $e(\cdot)$ denotes the symmetrized gradient.

Additionally, $\rho>0$ is the density of the elastic media and $\mathbf{f}$ is a volumic force acting on the structure (the gravity field, for example). In the incompressible case that we will study separately, the stress tensor writes

$$
\sigma\left(\mathbf{u}_{\varepsilon}, \eta_{\varepsilon}\right)=-\eta_{\varepsilon} \operatorname{Id}+2 \mu e\left(\mathbf{u}_{\varepsilon}\right)
$$

where $\eta_{\varepsilon}$ is the Lagrange multiplier, homogeneous to a pressure, associated with the additional incompressibility constraint $\operatorname{div} \mathbf{u}_{\varepsilon}=0$, and Id the identity operator. 
In both cases, initial conditions on the displacement and velocity complete the system. On the external boundary $\Gamma_{D}$, we impose no-slip boundary conditions on the displacement. On the external boundary $\Gamma_{N}$ and the internal alveolar boundaries $\Gamma_{\varepsilon}^{\mathbf{k}}$, we consider a pressure force exerted on the boundary. The external pressure $p_{N}$ is given, modeling the action of the diaphragm, whereas the pressures $p_{\varepsilon}^{\mathbf{k}}$, which denote the uniform air pressure inside each alveolus $\mathcal{Y}_{F, \varepsilon}^{\mathbf{k}}$, are unknowns and depend on the airflow through the dyadic tree, which we describe next.

\subsection{Poiseuille flow through a finite resistive dyadic tree}

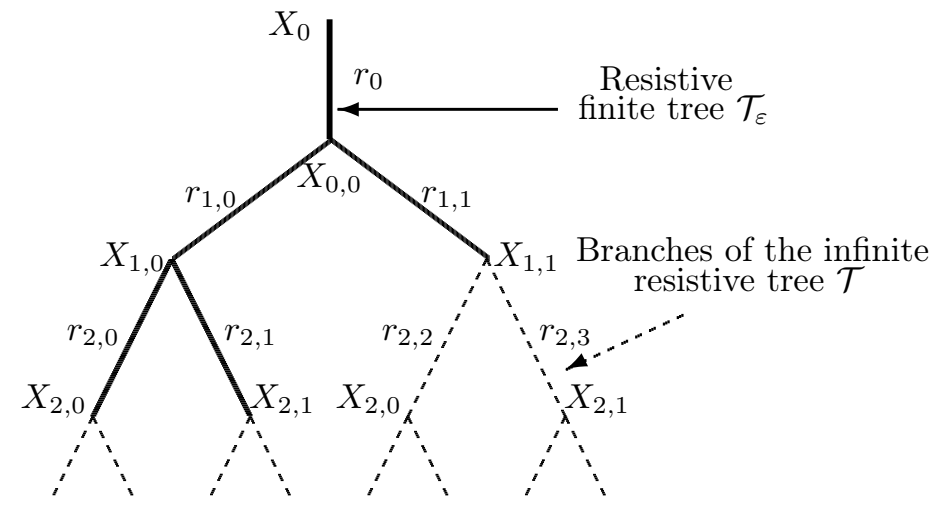

Figure 3: Resistive finite and infinite trees

We consider here the circulation of air through a tree of connected pipes. First, let us consider the flow of an incompressible, viscous, non-inertial fluid through a single pipe. According to Poiseuille's law, which we assume is valid in all branches, the flow rate $\Phi$ through the pipe is proportional to the pressure drop between its two end points, which is expressed by the equation

$$
P_{\text {in }}-P_{\text {out }}=r \Phi,
$$

where $r$ is the resistance of the pipe, a positive coefficient which varies with the fluid viscosity and with $L / D^{4}$, where $L$ is the length of the pipe and $D$ is its width. According to this description, we can fully characterize the airflow through the full dyadic tree $\mathcal{T}_{\varepsilon}$ by introducing a fixed sequence of resistances

$$
r_{n, k}>0 \quad \text { for } n \in \mathbb{N} \text { and } 0 \leq k \leq 2^{n}-1,
$$

which are associated with each edge of the trees $\mathcal{T}_{\varepsilon}$ and $\mathcal{T}$ as in Figure 3. We suppose that these resistances do not depend on the parameter $\varepsilon$. We call such trees resistive 
dyadic trees, and will still denote by $\mathcal{T}_{\varepsilon}$ and $\mathcal{T}$ the set of vertices and the associated resistances when no confusion is possible.

Given a set of pressures at the outlets, it is now possible to compute the corresponding set of fluxes thanks to Poiseuille's law, and vice versa. Let the pressure at the root node be denoted by $p_{e}$. Let

$$
\mathbf{p}_{\varepsilon}=\left(p_{\varepsilon}^{n, k}\right)_{n, k \in \mathcal{E}_{\varepsilon}} \quad \text { and } \quad \phi_{\varepsilon}=\left(\phi_{\varepsilon}^{n, k}\right)_{n, k \in \mathcal{E}_{\varepsilon}}
$$

be the pressure and flux vectors at the outlets of the tree, with the convention that the fluxes are considered positive when air is entering the tree through the corresponding outlet. Since Poiseuille's law is linear, there exists also a linear relation between $\mathbf{p}_{\varepsilon}$ and $\phi_{\varepsilon}$, i.e. a matrix $A^{\varepsilon}$ indexed by $\mathcal{E}_{\varepsilon}$ and such that

$$
\mathbf{p}_{\varepsilon}-p_{e} \mathbf{1}=A^{\varepsilon} \phi_{\varepsilon}
$$

where $\mathbf{1}$ is the vector $(1, \ldots, 1)$. The appearance of this unit vector follows from the observation that at rest, when there are no air fluxes in the tree, the air pressure is equal at all the outlets including the root node. Then the left-hand side of Eq. (1.10) must be zero.

Proposition 1.1. The matrix $A^{\varepsilon}$ is symmetric definite positive.

We do not give the details of the proof here, but we refer to [18] for a proof which extends readily to our setting. With a slight abuse of notation, we will also call $A^{\varepsilon}$ the same matrix reindexed by $\mathbf{Z}_{\varepsilon}^{\Omega}$ as an alternate numbering of the alveoli, with the convention that

$$
A_{\mathbf{k}, \mathbf{l}}^{\varepsilon}=A_{a, b}^{\varepsilon} \text { for } a=\omega_{\varepsilon}(\mathbf{k}) \text { and } b=\omega_{\varepsilon}(\mathbf{l}),
$$

where $\omega_{\varepsilon}$ is the one-to-one mapping defined in (1.4).

\subsection{Coupling the elastic structure and the resistive dyadic tree}

We are now in a position to write the fully coupled fluid-structure interaction model. We model the air in the alveoli as incompressible, so when the volume of the holes changes the air escapes through the tree. The pressures generated by this flow are related to the change in volume of the alveoli by (1.10).

We recall the convention that the flux is positive when air enters the tree through the outlet, so we obtain, at first order, the flux of air $\phi_{\varepsilon}^{\mathbf{k}}$ associated with the alveolus $\mathcal{Y}_{F, \varepsilon}^{\mathbf{k}}$ by the formula, see e.g. [3,8]:

$$
\phi_{\varepsilon}^{\mathbf{k}}=\int_{\Gamma_{\varepsilon}^{\mathbf{k}}} \partial_{t} \mathbf{u}_{\varepsilon} \cdot \mathbf{n}_{\varepsilon},
$$

where we recall that the normal vector $\mathbf{n}_{\varepsilon}$ points out into $\mathcal{Y}_{F, \varepsilon}^{\mathbf{k}}$. Moreover, using Eq. (1.10) and the matrix $A^{\varepsilon}$ introduced in (1.11), we can write the pressure $p_{\varepsilon}^{\mathbf{k}}$ 
inside each hole $\mathcal{Y}_{F, \varepsilon}^{\mathbf{k}}$ for $\mathbf{k} \in \mathbf{Z}_{\varepsilon}^{\Omega}$ as a function of the normal displacement field $\mathbf{u}_{\varepsilon}$ on the whole interior interface $\Gamma_{\varepsilon}$ :

$$
p_{\varepsilon}^{\mathbf{k}}-p_{e}=\sum_{\mathbf{l} \in \mathbf{Z}_{\varepsilon}^{\Omega}} A_{\mathbf{k}, 1}^{\varepsilon}\left(\int_{\Gamma_{\varepsilon}^{1}} \partial_{t} \mathbf{u}_{\varepsilon} \cdot \mathbf{n}_{\varepsilon}\right) .
$$

We now state two models we will analyze in the next two sections. In the first case we shall study, corresponding to a compressible structure, the displacement field $\mathbf{u}_{\varepsilon}$ satisfies the system:

$$
\left\{\begin{array}{rlrl}
\rho \partial_{t t} \mathbf{u}_{\varepsilon} & -\operatorname{div} \sigma\left(\mathbf{u}_{\varepsilon}\right)=\mathbf{f}, & \text { in } \Omega_{\varepsilon} \\
\sigma\left(\mathbf{u}_{\varepsilon}\right) \mathbf{n}_{\varepsilon} & =-\left(p_{e}+\sum_{\mathbf{l} \in \mathbf{Z}_{\varepsilon}^{\Omega}} A_{\mathbf{k}, \mathbf{l}}^{\varepsilon} \int_{\Gamma_{\varepsilon}^{1}} \partial_{t} \mathbf{u}_{\varepsilon} \cdot \mathbf{n}_{\varepsilon}\right) \mathbf{n}_{\varepsilon}, & & \text { on all } \Gamma_{\varepsilon}^{\mathbf{k}} \\
\sigma\left(\mathbf{u}_{\varepsilon}\right) \mathbf{n} & =-p_{N} \mathbf{n}, & & \text { on } \Gamma_{N} \\
\mathbf{u}_{\varepsilon} & =\mathbf{0}, & & \text { on } \Gamma_{D} \\
\mathbf{u}_{\varepsilon}(0) & =\mathbf{u}_{0}, \quad \partial_{t} \mathbf{u}_{\varepsilon}(0)=\mathbf{u}_{1}, & & \text { in } \Omega_{\varepsilon}
\end{array}\right.
$$

We recall that the stress tensor $\sigma\left(\mathbf{u}_{\varepsilon}\right)$ introduced in (1.7) reads

$$
\sigma\left(\mathbf{u}_{\varepsilon}\right)=\lambda \operatorname{div}\left(\mathbf{u}_{\varepsilon}\right) \operatorname{Id}+2 \mu e\left(\mathbf{u}_{\varepsilon}\right),
$$

and $\mathbf{u}_{0}, \mathbf{u}_{1}$ are initial conditions describing the state of the elastic media at $t=0$, chosen independently of the microscale parameter $\varepsilon$ and thus defined on the whole domain $\Omega$.

In the second case we shall study, corresponding to a linear incompressible structure, the unknowns of the model are the displacement field $\mathbf{u}_{\varepsilon}$ and the scalar-valued unknown $\eta_{\varepsilon}$, homogeneous to a pressure and satisfying:

$$
\left\{\begin{aligned}
\rho \partial_{t t} \mathbf{u}_{\varepsilon} & =\operatorname{div} \sigma\left(\mathbf{u}_{\varepsilon}, \eta_{\varepsilon}\right)=\mathbf{f}, & & \text { in } \Omega_{\varepsilon}, \\
\operatorname{div} \mathbf{u}_{\varepsilon} & =0, & & \text { in } \Omega_{\varepsilon}, \\
\sigma\left(\mathbf{u}_{\varepsilon}, \eta_{\varepsilon}\right) \mathbf{n}_{\varepsilon} & =-\left(p_{e}+\sum_{\mathbf{l} \in \mathbf{Z}_{\varepsilon}^{\Omega}} A_{\mathbf{k}, \mathbf{l}}^{\varepsilon} \int_{\Gamma_{\varepsilon}^{1}} \partial_{t} \mathbf{u}_{\varepsilon} \cdot \mathbf{n}_{\varepsilon}\right) \mathbf{n}_{\varepsilon}, & & \text { on all } \Gamma_{\varepsilon}^{\mathbf{k}}, \\
\sigma\left(\mathbf{u}_{\varepsilon}, \eta_{\varepsilon}\right) \mathbf{n} & =-p_{N} \mathbf{n}, & & \text { on } \Gamma_{N}, \\
\mathbf{u}_{\varepsilon} & =\mathbf{0}, & & \text { on } \Gamma_{D}, \\
\mathbf{u}_{\varepsilon}(0) & =\mathbf{u}_{0}, \quad \partial_{t} \mathbf{u}_{\varepsilon}(0)=\mathbf{u}_{1}, & & \text { in } \Omega_{\varepsilon},
\end{aligned}\right.
$$

where the stress tensor $\sigma\left(\eta_{\varepsilon}, \mathbf{u}_{\varepsilon}\right)$ introduced in (1.8) now reads:

$$
\sigma\left(\mathbf{u}_{\varepsilon}, \eta_{\varepsilon}\right)=-\eta_{\varepsilon} \operatorname{Id}+2 \mu e\left(\mathbf{u}_{\varepsilon}\right)
$$

Remark 1.3. This kind of averaged boundary conditions, non-local at the alveolar level, appears in other fluid-structure models, such as in the static foam model 
studied in [3] or when studying vibrations of rigid tubes immersed in a fluid [2]. Here we deal with a special case where the boundary conditions are also globally coupled by the matrix $A^{\varepsilon}$, so in addition to being non-local at the microscopic level there is a non-local coupling at the macroscopic level between the alveoli.

\section{Resistance operators: coupling domain and resistive dyadic tree}

Our goal in this paper is to perform the asymptotic analysis of the problems (1.14) and (1.15) as the parameter $\varepsilon$ goes to zero and the number of generations of the tree goes to infinity. The relation (1.10) describes the action of the tree at a discrete level, using a matrix $A^{\varepsilon}$ whose dimension depends on $\varepsilon$. We are going to introduce an equivalent description at the continuous level, in order to effectively study the coupling between the airflow in the tree and the displacement of the structure in a fixed setting, as a linear operator on $L^{2}(\Omega)$.

\subsection{Definition}

Recall that thanks to (1.5), we have a one-to-one mapping between the leafs of $\mathcal{T}_{\varepsilon}$ and the alveoli $\mathcal{Y}_{F, \varepsilon}^{\mathbf{k}}$ which allows us to identify the outlets of the resistive dyadic tree and the alveoli. This motivates the introduction of the following projection operator, recalling that $|\mathcal{Y}|=1$ :

Definition 2.1. Let $\Pi_{\varepsilon}$ be the $L^{2}$-projector on the set of functions taking constant values on each cell $\mathcal{Y}_{\varepsilon}^{\mathbf{k}}$ for $\mathbf{k} \in \mathbf{Z}_{\varepsilon}^{\Omega \text { : }}$

$$
\Pi_{\mathcal{\varepsilon}}(q)=\sum_{\mathbf{k} \in \mathbf{Z}_{\varepsilon}^{\Omega}} \varepsilon^{-d}\left(\int_{\mathcal{Y}_{\varepsilon}^{\mathbf{k}}} q\right) \chi_{\varepsilon}^{\mathbf{k}} .
$$

Given a continuous flux function $\phi_{\varepsilon} \in L^{2}(\Omega)$, we define a vector of discrete fluxes as

$$
\phi_{\varepsilon}=\left(q_{\varepsilon}^{\mathbf{k}}\right)_{\mathbf{k} \in \mathbf{Z}_{\varepsilon}^{\Omega}}, \quad \text { with } \phi_{\varepsilon}^{\mathbf{k}}=\int_{\mathcal{Y}_{\varepsilon}^{\mathbf{k}}} \phi_{\varepsilon} .
$$

The corresponding pressure vector is defined as

$$
\mathbf{p}_{\varepsilon}=\left(p_{\varepsilon}^{\mathbf{k}}\right)_{\mathbf{k} \in \mathbf{Z}_{\varepsilon}^{\Omega}}=A^{\varepsilon} \boldsymbol{\phi}_{\varepsilon},
$$

where $A^{\varepsilon}$ is the matrix indexed by $\mathbf{Z}_{\varepsilon}^{\Omega}$ defined in (1.11). Finally we introduce the pressure function $p_{\varepsilon} \in L^{2}(\Omega)$ as the function which takes constant value $p_{\varepsilon}^{\mathbf{k}}$ in each cell $\mathcal{Y}_{\varepsilon}^{\mathbf{k}}$ for $\mathbf{k} \in \mathbf{Z}_{\varepsilon}^{\Omega}$, and 0 elsewhere. We denote by $\mathcal{R}_{\varepsilon}$ the following operator

$$
\begin{cases}L^{2}(\Omega) & \rightarrow L^{2}(\Omega), \\ \phi_{\varepsilon} & \mapsto p_{\varepsilon} .\end{cases}
$$

Now, using the resistance operator $\mathcal{R}_{\varepsilon}$ we can rewrite the problem (1.14) with a new expression of the non-local boundary conditions on the boundary of the holes: 


$$
\left\{\begin{aligned}
\rho \partial_{t t} \mathbf{u}_{\varepsilon} & =\operatorname{div} \sigma\left(\mathbf{u}_{\varepsilon}\right)=\mathbf{f}, & & \text { in } \Omega_{\varepsilon}, \\
\sigma\left(\mathbf{u}_{\varepsilon}\right) \mathbf{n}_{\varepsilon} & =-\left(p_{e}+\partial_{t} \mathcal{R}_{\varepsilon} \operatorname{div} \mathbf{u}_{\varepsilon}\right) \mathbf{n}_{\varepsilon}, & & \text { on all } \Gamma_{\varepsilon}^{\mathbf{k}}, \\
\sigma\left(\mathbf{u}_{\varepsilon}\right) \mathbf{n} & =-p_{N} \mathbf{n}, & & \text { on } \Gamma_{N}, \\
\mathbf{u}_{\varepsilon} & =\mathbf{0}, & & \text { on } \Gamma_{D}, \\
\mathbf{u}_{\varepsilon}(0) & =\mathbf{u}_{0}, \quad \partial_{t} \mathbf{u}_{\varepsilon}(0)=\mathbf{u}_{1}, & & \text { in } \Omega_{\varepsilon} .
\end{aligned}\right.
$$

A similar strong system of equations can easily be derived in the incompressible case from problem (1.15). In order to study the asymptotic properties of the solutions of problem (2.4), let us detail some useful properties of the operator $\mathcal{R}_{\varepsilon}$.

Proposition 2.1. The resistance operator $\mathcal{R}_{\varepsilon}$ can be expressed as a kernel integral operator:

$$
p_{\varepsilon}=\mathcal{R}_{\varepsilon} \phi_{\varepsilon} \quad \Longleftrightarrow \quad p_{\varepsilon}\left(\mathbf{x}_{1}\right)=\int_{\Omega} K_{\varepsilon}\left(\mathbf{x}_{1}, \mathbf{x}_{2}\right) \phi_{\varepsilon}\left(\mathbf{x}_{2}\right) \mathrm{d} \mathbf{x}_{2} \quad \forall \mathbf{x}_{1} \in \Omega,
$$

where $K_{\varepsilon} \in L^{1}(\Omega \times \Omega)$ is a piecewise constant function defined as:

$$
K_{\varepsilon}\left(\mathbf{x}_{1}, \mathbf{x}_{2}\right)= \begin{cases}A_{\mathbf{k}, 1}^{\varepsilon} & \text { if } \mathbf{x}_{1} \in \mathcal{Y}_{\varepsilon}^{\mathbf{k}}, \mathbf{x}_{2} \in \mathcal{Y}_{\varepsilon}^{\mathbf{l}} \text { for some } \mathbf{k} \in \mathbf{Z}_{\varepsilon}^{\Omega}, \mathbf{l} \in \mathbf{Z}_{\varepsilon}^{\Omega}, \\ 0 & \text { elsewhere. }\end{cases}
$$

Remark 2.1. The operator $\mathcal{R}_{\varepsilon}$ sums up the interaction of the tree with the structure, acting as a Dirichlet to Neumann operator by relating the rate of compression of the structure to the pressure forces on the boundary of the holes in a non-local way.

Note that since the matrix $A^{\varepsilon}$ is symmetric and positive, these properties are transposed to the operator $\mathcal{R}_{\varepsilon}$ : for all $\phi, \psi$ in $L^{2}(\Omega)$ we have

$$
\int_{\Omega}\left(\mathcal{R}_{\varepsilon} \phi\right) \psi=\int_{\Omega}\left(\mathcal{R}_{\varepsilon} \psi\right) \phi \quad \text { and } \quad \int_{\Omega}\left(\mathcal{R}_{\varepsilon} \phi\right) \phi \geq 0 .
$$

As a consequence, the following Cauchy-Schwartz inequality holds: for all $\phi, \psi$ in $L^{2}(\Omega)$

$$
\left|\int_{\Omega}\left(\mathcal{R}_{\varepsilon} \phi\right) \psi\right|^{2} \leq\left(\int_{\Omega}\left(\mathcal{R}_{\varepsilon} \phi\right) \phi\right)\left(\int_{\Omega}\left(\mathcal{R}_{\varepsilon} \psi\right) \psi\right)
$$

In order to give a better understanding of the nature of the operator $\mathcal{R}_{\varepsilon}$ and its kernel $K_{\varepsilon}$, we recall here the construction presented in [39]. Each airway of the bronchial tree, represented by an edge of the infinite dyadic tree $\mathcal{T}$, say $X_{n, k}$, irrigates a portion of the parenchyma, which we denote by $\Omega_{n, k} \subset \Omega$. Moreover, for a given $\varepsilon>0$ each end indexed by $(n, k) \in \mathcal{E}_{\varepsilon}$ of the finite tree $\mathcal{T}_{\varepsilon}$ is associated to a terminal airway. Thus $\Omega_{n, k}$ should contain exactly one cell $\mathcal{Y}_{\varepsilon}^{\mathbf{k}}$ so as to define a one-too-one mapping $(n, k)=\omega_{\varepsilon}(\mathbf{k})$ as in (1.4). This irrigation process follows a hierarchical structure, and induces a sequence of domain decompositions satisfying the following properties: 
Definition 2.2. Multi-scale dyadic decompositions of the domain $\Omega$

Let $\left(\Omega_{n, k}\right)_{n>0, k=0, \ldots, 2^{n}-1}$ be a sequence of open non-empty connected subsets of $\Omega$. We say that $\mathcal{O}=\left(\Omega_{n, k}\right)_{n \geq 0, k=0, \ldots, 2^{n}-1}$ is a multi-scale decomposition of $\Omega$ if for every $n \geq 0$,

(1) $\bigcup_{k=0}^{2^{n}-1} \bar{\Omega}_{n, k}=\bar{\Omega}$,

(2) $\Omega_{n, j} \cap \Omega_{n, k}=\emptyset$ as soon as $j \neq k$,

(3) $\bar{\Omega}_{n+1,2 k} \cup \bar{\Omega}_{n+1,2 k+1}=\bar{\Omega}_{n, k}$, for all $k \in\left\{0, \ldots, 2^{n}-1\right\}$.

Then we have the following result, which can be verified as in the onedimensional case [18]:

Proposition 2.2. The kernel $K_{\varepsilon}$ writes

$$
K_{\varepsilon}\left(\mathbf{x}_{1}, \mathbf{x}_{2}\right)=\sum_{(n, k) \in \mathcal{I}_{\varepsilon} \cup \mathcal{E}_{\varepsilon}} r_{n, k} \chi_{n}^{k}\left(\mathbf{x}_{1}\right) \chi_{n}^{k}\left(\mathbf{x}_{2}\right), \quad \text { for } \mathbf{x}_{1}, \mathbf{x}_{2} \text { in } \Omega,
$$

where $\chi_{n}^{k}$ is the indicator function of the subdomain $\Omega_{n, k}$ and the sets of indexes $\mathcal{E}_{\varepsilon}$ and $\mathcal{I}_{\varepsilon}$ are defined by (1.3).

\subsection{Asymptotic study: an example}

The convergence properties of $\mathcal{R}_{\varepsilon}$ as $\varepsilon$ goes to zero are the key to understanding the asymptotic behavior of the solutions of problem (2.4). Some of these properties have been studied extensively in the 1D case in [18] and in a more generic setting in [39]. For the sake of simplicity, we present here a particular construction which is based on the symmetric model of the bronchial tree developed by Weibel [40] and allows us to extend to our multi-dimensional setting the results obtained in [18].

\subsubsection{Geometry: connecting the domain to the tree}

We consider a square or cubic domain $\Omega=(0,1)^{d}, d=2$ or 3 , and a lattice $\mathbf{Z}=\mathbb{Z}^{d}$ associated with a unit square or cubic periodic cell $\mathcal{Y}=(0,1)^{d}$. Clearly, for any integer $n \geq 0$, the domain $\Omega$ can be tiled by $2^{d n}$ translated copies of $\mathcal{Y}$ rescaled by a factor $2^{-n}$. To organize these cells in a multi-scale decomposition, we suppose that we are given a bifurcating space-filling tree in $\Omega$ which is a geometrical representation of a dyadic resistive tree $\mathcal{T}$.

Building a space-filling tree Space-filling bifurcating trees are geometrical objects, which can be constructed by recursion, whose canopy (the branch tips) becomes dense in $\Omega$, see e.g. the $H$-tree depicted in Figure 4 or other examples in [26]. To comply with the periodic geometry, we propose the following construction for a square, which can be readily extended to the cube.

One starts with a "trunk", a segment joining a point located on the border of the square $\Omega$, for example $(0,1 / 2)$, to the center of $\Omega$ at $(1 / 2,1 / 2)$ which is a fertile "bud". This bud then generates two branches, on which only two terminal buds are 


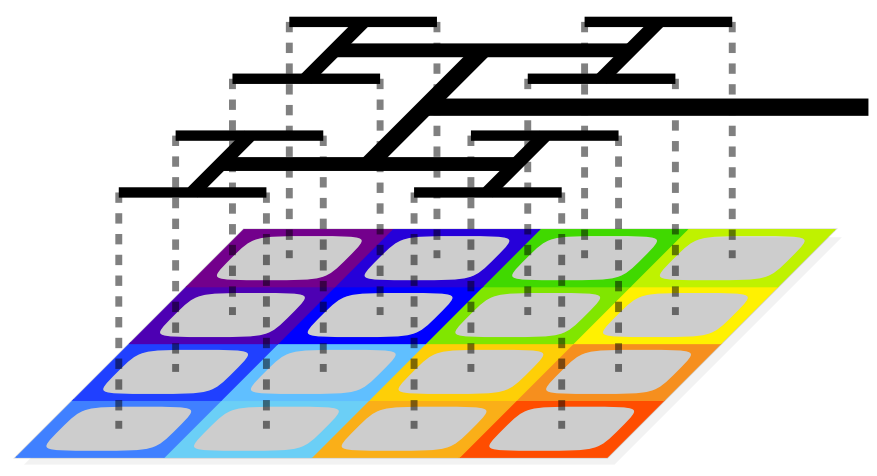

Figure 4: Perforated square domain $\Omega_{n}$ connected by a $\mathrm{H}-$ tree, for $n=2$

fertile. These buds again generate each two branches, on which one has now four terminal buds which are located at the centers of the four squares paving $\Omega$. One can then repeat the process for each little square, and so on ad infinitum.

By construction, the buds obtained after an even number $2 n$ of bifurcations are located at the center of the square cells $\mathcal{Y}_{\varepsilon}^{\mathbf{k}}$ for $\varepsilon=2^{-n}$ (or a number $3 n$ of bifurcations for the three-dimensional cubes). Note that the branches of the tree are bounded in length, but become infinite in number.

Building the multi-scale decomposition The next step is to use the spacefilling tree to build a multi-scale decomposition $\mathcal{O}$ of the square $\Omega$ such that for each $n \geq 0$, the subdomains $\Omega_{d n, k}$ coincide with a rescaled periodic cell. To do this, we simply associate each bud of the tree obtained after $d n$ bifurcations and the unique cell $\mathcal{Y}_{\varepsilon}^{\mathbf{k}}$ with $\varepsilon=2^{-n}$ located by construction around this bud. Then, using the correspondence between the space-filling tree and the abstract dyadic tree $\mathcal{T}$, we create a one-to-one mapping, noted $\omega_{n}$, between the cells $\mathcal{Y}_{\varepsilon}^{\mathbf{k}}$ indexed by $\mathbf{k}$ in $\mathbf{Z}_{\varepsilon}^{\Omega}$ for $\varepsilon=2^{-n}$, and the nodes $X_{d n, k}$, indexed by $k$ in $0 \leq k \leq 2^{d n}-1$. This allows us to define the subdomains of generation $d n$ of $\mathcal{O}$ as:

$$
\Omega_{d n, k}=\mathcal{Y}_{\varepsilon}^{\mathbf{k}} \text { for } k=\omega_{n}(\mathbf{k}), \text { for } \mathbf{k} \in \mathbf{Z}_{\varepsilon}^{\Omega} \text {, with } \varepsilon=2^{-n} .
$$

Subdomains belonging to an intermediate generation, e.g. $m$ with $d n<m<d(n+1)$ for $n \geq 0$, are then defined recursively by the relation

$$
\Omega_{m, k}=\operatorname{Interior}\left(\overline{\Omega_{m+1,2 k}} \cup \overline{\Omega_{m+1,2 k+1}}\right)
$$

Reparameterization In this example, the parameter $\varepsilon>0$ is only allowed to take the values $2^{-n}$ for positive integers $n$. For simplicity, we will index in this example the variables and unknowns by $n$ instead of $\varepsilon: \Omega_{\varepsilon} \equiv \Omega_{n}, \mathcal{R}_{\varepsilon} \equiv \mathcal{R}_{n}$, etc. 


\subsubsection{Definition and convergence of the resistance operators}

To completely define the resistance operator $\mathcal{R}_{n}$ we also have to describe the resistances of the tree. In particular, we are interested in conditions on these resistances implying the convergence of the sequence $K_{n}$ in an appropriate space. Note that $\mathcal{T}_{n}$ is a dyadic tree with $d n$ full generations. By Prop. 2.2 the kernel $K_{n}$ associated with $\mathcal{R}_{n}$ is defined by:

$$
K_{n}\left(\mathbf{x}_{1}, \mathbf{x}_{2}\right)=\sum_{N=0}^{d n} \sum_{k=0}^{2^{N}-1} r_{n, k} \chi_{N}^{k}\left(\mathbf{x}_{1}\right) \chi_{N}^{k}\left(\mathbf{x}_{2}\right), \quad \text { for } \mathbf{x}_{1}, \mathbf{x}_{2} \text { in } \Omega .
$$

We also define the limit kernel $K$ associated with the infinite resistive tree $\mathcal{T}$ and the dyadic multi-scale decomposition $\mathcal{O}$ as

$$
K\left(\mathbf{x}_{1}, \mathbf{x}_{2}\right)= \begin{cases}\sum_{N=0}^{+\infty} \sum_{k=0}^{2^{N}-1} r_{n, k} \chi_{N}^{k}\left(\mathbf{x}_{1}\right) \chi_{N}^{k}\left(\mathbf{x}_{2}\right) & \text { if } \mathbf{x}_{1} \neq \mathbf{x}_{2} \\ 0 & \text { else }\end{cases}
$$

Note that the sum in (2.11) is in fact finite since $\mathbf{x}_{1}$ and $\mathbf{x}_{2}$ eventually belong to different cells of the multi-scale decomposition.

We consider in particular the case where the resistances of the tree are regular and follow a geometric law.

Definition 2.3. A resistive dyadic tree is called regular if all resistances associated with branches located at a common generation of the tree have a common value, i.e. there exists a sequence $\left(r_{n}\right)_{n \geq 0}$ with $r_{n}>0$ such that:

$$
r_{n, k}=r_{n}, \quad \text { for all } n \geq 0, \quad k \in\left\{0, \cdots, 2^{n}-1\right\} .
$$

A regular resistive dyadic tree is called geometric if there exists a real constant $\alpha>0$ such that:

$$
r_{n}=r_{0} \alpha^{n} \quad \text { for all } n \geq 0 .
$$

Remark 2.2. Measures by Weibel [40] show that the resistances in the human bronchial tree are approximately geometric, with the scaling factor $\alpha \approx 1.63$.

Note that the difference $K_{n}-K$ is in this case similar to the remainder of a geometric sum. Extending to the multi-dimensional setting the arguments presented in the proof of Prop. 1.1 in [18], it is straightforward to obtain the following convergence properties for the sequence of operators $\mathcal{R}_{n}$ :

Proposition 2.3. In general, if the resistances of the tree satisfy the condition

$$
\sum_{n=0}^{+\infty} \frac{1}{2^{n}} \max _{k \in\left\{0, \cdots, 2^{n}-1\right\}} r_{n, k}<\infty
$$

then:

- $K_{n}$ converges to $K$ in $L^{\infty}\left(\Omega, L^{1}(\Omega)\right)$, 
- $\mathcal{R}_{n}$ converges to an operator $\mathcal{R}$ in $\mathcal{L}\left(L^{2}(\Omega), L^{2}(\Omega)\right)$, which is also an integral operator with associated kernel $K$.

In particular, if the resistive dyadic tree is regular and geometric with scaling factor $0<\alpha<2$ then the previous convergences hold and:

$$
\left\|\mathcal{R}-\mathcal{R}_{n}\right\|_{\mathcal{L}\left(L^{2}(\Omega)\right)}=\frac{r_{0}}{1-\alpha / 2}\left(\frac{\alpha}{2}\right)^{d n+1} .
$$

\subsection{General case and abstract convergence condition}

In the setting of this explicit construction, the sequence of operator $\left(\mathcal{R}_{n}\right)_{n \geq 0}$ converge strongly. We propose to extend this observation to the more generic setting described in Section 1 as the following abstract condition:

$$
\begin{aligned}
& \text { There exists } \mathcal{R} \in \mathcal{L}\left(L^{2}(\Omega) ; L^{2}(\Omega)\right) \text { such that } \\
& \mathcal{R}_{\varepsilon} \rightarrow \mathcal{R} \text { strongly in } \mathcal{L}\left(L^{2}(\Omega) ; L^{2}(\Omega)\right),
\end{aligned}
$$

where $\mathcal{L}\left(L^{2}(\Omega) ; L^{2}(\Omega)\right)$ is equipped with the strong topology associated with the operator norm. In particular, we show in this paper that this condition is sufficient in order to derive rigorously homogenized models corresponding to (1.14) and (1.15).

Remark 2.3. An immediate, but important consequence of the condition (2.12) is that the limit resistance operator $\mathcal{R}$ is a compact and positive operator in $L^{2}(\Omega)$, since the operators $\mathcal{R}_{\varepsilon}$ are definite positive and have finite rank.

Remark 2.4. The results of Prop. 2.3 are a direct consequence of a particular property of the construction proposed in Section 2.2: for any $n \geq 0$, the periodic paving of the domain $\Omega$ by the cells $\mathcal{Y}_{n}^{\mathbf{k}}$ matches exactly the decomposition of the domain induced by the subdomains $\Omega_{d n, k}$, see (2.9). This is not true for any multiscale decomposition, or possible for any periodic cell (e.g. for an hexagon). Much more generic conditions on a given multi-scale decomposition and set of resistances, sufficient to build a sequence of operators $\mathcal{R}_{\varepsilon}$ strongly converging to the associated operator $\mathcal{R}$ will be described and studied in a forthcoming paper, see also [6].

\section{Study in the compressible case: homogenization limit}

Let us now turn to the asymptotic analysis of the problem (2.4) under the condition (2.12). We will proceed in several steps: first, we prove that (2.4) is well-posed and we derive a priori estimates independent of the parameter $\varepsilon$. Then, we study the limit as $\varepsilon$ goes to zero, and we obtain the two-scale problem and rigorously justify the asymptotic limit in a single step thanks to the two-scale convergence method. Finally, we study the limit problem and describe its specificities, and we show that it is well-posed. 


\subsection{Variational formulation and a priori estimates}

In this part, we write the variational formulation associated with problem (2.4) and derive an existence and uniqueness result for it. Then we introduce an extension operator for functions defined on the parameter-dependent domain $\Omega_{\varepsilon}$ to functions defined on the fixed domain $\Omega$ that allows us to obtain a priori estimates independent of $\varepsilon$ on the weak solutions of (2.4).

\subsubsection{Variational formulation}

Let $\mathbf{V}_{\varepsilon}=\left\{\mathbf{v} \in \mathbf{H}^{1}\left(\Omega_{\varepsilon}\right)|\mathbf{v}|_{\Gamma_{D}}=\mathbf{0}\right\}$ and $\mathbf{V}=\left\{\mathbf{v} \in \mathbf{H}^{1}(\Omega)|\mathbf{v}|_{\Gamma_{D}}=\mathbf{0}\right\}$ where $\left.\mathbf{v}\right|_{\Gamma_{D}}$ denotes the trace of $\mathbf{v}$ on $\Gamma_{D}$, and $\mathbf{X}_{\varepsilon}=\mathbf{L}^{2}\left(\Omega_{\varepsilon}\right), \mathbf{X}=\mathbf{L}^{2}(\Omega)$. Let us denote by $\|\cdot\|_{0, \Omega_{\varepsilon}}$ and $\|\cdot\|_{0, \Omega}$ the respective $L^{2}$-norms in $\Omega_{\varepsilon}$ and $\Omega$, and by $|\cdot|_{1, \Omega_{\varepsilon}}=\|\nabla(\cdot)\|_{0, \Omega_{\varepsilon}}$ and $|\cdot|_{1, \Omega}=\|\nabla(\cdot)\|_{0, \Omega}$ the respective $H^{1}$ Sobolev semi-norms.

Let $T>0$ be an arbitrary time. We make the following assumptions on the data:

$$
\mathbf{f} \in \mathbf{L}^{2}((0, T) \times \Omega), \quad p_{N}, p_{e} \in H^{1}(0, T), \quad \mathbf{u}_{0} \in \mathbf{V} \text { and } \mathbf{u}_{1} \in \mathbf{L}^{2}(\Omega) .
$$

We obtain a variational formulation associated to (2.4) by using test functions in $\mathbf{V}_{\varepsilon}$ : find $\mathbf{u}_{\varepsilon} \in L^{\infty}\left(0, T ; \mathbf{V}_{\varepsilon}\right)$ with $\partial_{t} \mathbf{u}_{\varepsilon} \in L^{\infty}\left(0, T ; \mathbf{X}_{\varepsilon}\right)$ such that:

$$
\left\{\begin{array}{c}
\text { For all } \mathbf{v}_{\varepsilon} \in \mathbf{V}_{\varepsilon}, \\
\frac{\mathrm{d}}{\mathrm{d} t}\left(\rho \partial_{t} \mathbf{u}_{\varepsilon}, \mathbf{v}_{\varepsilon}\right)_{0, \Omega_{\varepsilon}}+\frac{\mathrm{d}}{\mathrm{d} t} r_{\varepsilon}\left(\mathbf{u}_{\varepsilon}, \mathbf{v}_{\varepsilon}\right)+a_{\varepsilon}\left(\mathbf{u}_{\varepsilon}, \mathbf{v}_{\varepsilon}\right)=\ell_{\varepsilon}\left(\mathbf{v}_{\varepsilon}\right), \quad \text { in } \mathcal{D}^{\prime}(0, T), \\
\mathbf{u}_{\varepsilon}(0)=\mathbf{u}_{0}, \partial_{t} \mathbf{u}_{\varepsilon}(0)=\mathbf{u}_{1},
\end{array}\right.
$$

where $(\cdot, \cdot)_{0, \Omega_{\varepsilon}}$ is the $L^{2}$-scalar product in $\mathbf{X}_{\varepsilon}, a_{\varepsilon}(\cdot, \cdot): \mathbf{V}_{\varepsilon} \times \mathbf{V}_{\varepsilon} \rightarrow \mathbb{R}$ and $r_{\varepsilon}(\cdot, \cdot): \mathbf{V}_{\varepsilon} \times \mathbf{V}_{\varepsilon} \rightarrow \mathbb{R}$ are the bilinear forms defined by:

$$
\begin{aligned}
a_{\varepsilon}\left(\mathbf{u}_{\varepsilon}, \mathbf{v}_{\varepsilon}\right) & =\int_{\Omega_{\varepsilon}} \lambda \operatorname{div}\left(\mathbf{u}_{\varepsilon}\right) \operatorname{div}\left(\mathbf{v}_{\varepsilon}\right)+2 \mu e\left(\mathbf{u}_{\varepsilon}\right): e\left(\mathbf{v}_{\varepsilon}\right), \\
r_{\varepsilon}\left(\mathbf{u}_{\varepsilon}, \mathbf{v}_{\varepsilon}\right) & =\sum_{\mathbf{k}, \mathbf{l} \in \mathbf{Z}_{\varepsilon}^{\Omega}} A_{\mathbf{k}, \mathbf{l}}^{\varepsilon}\left(\int_{\Gamma_{\varepsilon}^{\mathbf{k}}} \mathbf{u}_{\varepsilon} \cdot \mathbf{n}_{\varepsilon}\right)\left(\int_{\Gamma_{\varepsilon}^{1}} \mathbf{v}_{\varepsilon} \cdot \mathbf{n}_{\varepsilon}\right) \\
& =\int_{0}^{t} \int_{\Omega} \frac{\partial}{\partial t} \mathcal{R}_{\varepsilon}\left(\chi_{F, \varepsilon} \operatorname{div} \widehat{\mathbf{u}_{\varepsilon}}\right) \frac{\partial}{\partial t} \Pi_{\varepsilon}\left(\chi_{F, \varepsilon} \operatorname{div} \widehat{\mathbf{v}_{\varepsilon}}\right),
\end{aligned}
$$

with the projector $\Pi_{\varepsilon}$ defined by (2.1), and finaly $\ell_{\varepsilon}(\cdot): \mathbf{V}_{\varepsilon} \rightarrow \mathbb{R}$ is the linear form

$$
\ell_{\varepsilon}\left(\mathbf{v}_{\varepsilon}\right)=\int_{\Omega_{\varepsilon}} \mathbf{f} \cdot \mathbf{v}_{\varepsilon}+\int_{\Gamma_{N}} p_{N} \mathbf{v}_{\varepsilon} \cdot \mathbf{n}-\int_{\Gamma_{\varepsilon}} p_{e} \mathbf{v}_{\varepsilon} \cdot \mathbf{n}_{\varepsilon} .
$$

For a given $\varepsilon>0$, this problem has been studied in [38], Section 6.2. The variational formulation (3.2) holds at least in $H^{-1}(0, T)$, and the initial conditions have a meaning in a weak sense under the assumptions of Prop. 3.1 below. We have the following existence and uniqueness result: 
Proposition 3.1. Under hypothesis (3.1), the variational problem (3.2) has a unique solution $\mathbf{u}_{\varepsilon}$ with:

$$
\mathbf{u}_{\varepsilon} \in L^{\infty}\left(0, T ; \mathbf{V}_{\varepsilon}\right), \quad \text { and } \quad \partial_{t} \mathbf{u}_{\varepsilon} \in L^{\infty}\left(0, T ; \mathbf{X}_{\varepsilon}\right) .
$$

The proof of existence and uniqueness is given in details in [38] and follows the classical Faedo-Galerkin method, see $[24,25]$ for reference. The key of the proof is the coercivity of the symmetric bilinear form $a_{\varepsilon}$, which results from the fact that $\lambda, \mu>0$ and that the Poincaré' and Korn's inequalities hold in $\mathbf{V}_{\varepsilon}$ since $\left|\Gamma_{D}\right|>0$ (see [11]), and also the positivity of the term associated to $\mathcal{R}_{\varepsilon}$.

\subsubsection{Extension operator and a priori estimates}

Because $\mathbf{u}_{\varepsilon}$ is defined on the porous domain $\Omega_{\varepsilon}$ and belongs to the $\varepsilon$-dependent space $\mathbf{V}_{\varepsilon}$, the estimates derived in [38] or the one we could compute directly from (3.2) depend a priori on $\varepsilon$. We are going to prove a priori bounds that are uniform in $\varepsilon$. As is standard when dealing with porous multiscale domains, we introduce an extension operator $\widehat{\cdot}$ from functions defined on $\Omega_{\varepsilon}$ to functions defined on $\Omega$. In the remainder of this section, $C$ will denote a constant independent of $\varepsilon$ unless otherwise explicitly specified.

Lemma 3.1. There exists a linear continuous extension operator denoted by $\widehat{\cdot}$ belonging to the space $\mathcal{L}\left(L^{\infty}\left(0, T ; \mathbf{H}^{k}\left(\Omega_{\varepsilon}\right)\right) ; L^{\infty}\left(0, T ; \mathbf{H}^{k}(\Omega)\right)\right)$ for $k=1,2$ such that for any $\mathbf{v}_{\varepsilon} \in L^{\infty}\left(0, T ; \mathbf{H}^{k}\left(\Omega_{\varepsilon}\right)\right) \cap W^{1, \infty}\left(0, T ; \mathbf{L}^{2}\left(\Omega_{\varepsilon}\right)\right)$,

(1) $\widehat{\mathbf{v}_{\varepsilon}}=\mathbf{v}_{\varepsilon}$ a.e. in $\Omega_{\varepsilon} \times(0, T)$,

(2) $\widehat{\partial_{t} \mathbf{v}_{\varepsilon}}=\partial_{t}\left(\widehat{\mathbf{v}_{\varepsilon}}\right)$ a.e. in $\Omega_{\varepsilon} \times(0, T)$,

(3) $\left\|\widehat{\mathbf{v}_{\varepsilon}}\right\|_{\mathbf{L}^{2}(\Omega)} \leq C\left\|\mathbf{v}_{\varepsilon}\right\|_{\mathbf{L}^{2}\left(\Omega_{\varepsilon}\right)}$ a.e. in $(0, T)$,

(4) $\left\|\widehat{\partial_{t} \mathbf{v}_{\varepsilon}}\right\|_{\mathbf{L}^{2}(\Omega)} \leq C\left\|\partial_{t} \mathbf{v}_{\varepsilon}\right\|_{\mathbf{L}^{2}\left(\Omega_{\varepsilon}\right)}$ a.e. in $(0, T)$,

(5) $\left\|\nabla\left(\widehat{\mathbf{v}_{\varepsilon}}\right)\right\|_{L^{2}(\Omega)} \leq C\left\|\nabla \mathbf{v}_{\varepsilon}\right\|_{L^{2}\left(\Omega_{\varepsilon}\right)}$ a.e. in $(0, T)$,

(6) Moreover, if $\mathbf{v}_{\varepsilon} \in \mathbf{V}_{\varepsilon}$, a.e. in $(0, T)$,

$$
\left\|e\left(\widehat{\mathbf{v}_{\varepsilon}}\right)\right\|_{L^{2}(\Omega)} \leq C\left\|e\left(\mathbf{v}_{\varepsilon}\right)\right\|_{L^{2}\left(\Omega_{\varepsilon}\right)} .
$$

Proof. The proof is the same as for Lemma 2.1 in [9].

We are now in a position to obtain uniform a priori estimates for the sequence $\left(\widehat{\mathbf{u}_{\varepsilon}}\right)_{\varepsilon>0}$, defined on the fixed space $\mathbf{V}$ :

Proposition 3.2. Under hypothesis (3.1), the sequence of solutions $\mathbf{u}_{\varepsilon}$ of problem (2.4) is such that

$$
\left|\widehat{\mathbf{u}_{\varepsilon}}\right|_{1, \Omega}^{2}+\left\|\widehat{\partial_{t} \mathbf{u}_{\varepsilon}}\right\|_{0, \Omega}^{2}+\mathcal{V}_{\varepsilon}(t) \leq C, \quad \text { a.e. } t \in(0, T),
$$

where

$$
\mathcal{V}_{\varepsilon}(t)=\int_{0}^{t} \int_{\Omega} \frac{\partial}{\partial t} \mathcal{R}_{\varepsilon}\left(\chi_{F, \varepsilon} \operatorname{div} \widehat{\mathbf{u}_{\varepsilon}}\right) \frac{\partial}{\partial t} \Pi_{\varepsilon}\left(\chi_{F, \varepsilon} \operatorname{div} \widehat{\mathbf{u}_{\varepsilon}}\right) .
$$


Remark 3.1. The quantity $\mathcal{V}_{\varepsilon}$ measures the dissipation of energy by the viscous forces in the bronchial tree. It is positive, since the operator $\mathcal{R}_{\varepsilon}$ is positive.

Proof. Let us describe the main ideas of the proof of (3.7) by taking formally $\mathbf{v}_{\varepsilon}=\partial_{t} \mathbf{u}_{\varepsilon}$ as a test function in (2.4). We get the following identity:

$$
\left\{\begin{aligned}
\frac{1}{2} \frac{\mathrm{d}}{\mathrm{d} t}\left(\rho\left\|\partial_{t} \mathbf{u}_{\varepsilon}\right\|_{0, \Omega_{\varepsilon}}^{2}+a\left(\mathbf{u}_{\varepsilon}, \mathbf{u}_{\varepsilon}\right)\right) \\
\quad+\int_{0}^{t} \int_{\Omega} \frac{\partial}{\partial t} \mathcal{R}_{\varepsilon}\left(\chi_{F, \varepsilon} \operatorname{div} \widehat{\mathbf{u}_{\varepsilon}}\right) \frac{\partial}{\partial t} \Pi_{\varepsilon}\left(\chi_{F, \varepsilon} \operatorname{div} \widehat{\mathbf{u}_{\varepsilon}}\right) \\
=\int_{\Omega_{\varepsilon}} \mathbf{f} \cdot \partial_{t} \mathbf{u}_{\varepsilon}+\int_{\Gamma_{N}} p_{N} \partial_{t} \mathbf{u}_{\varepsilon} \cdot \mathbf{n}-\sum_{\mathbf{k} \in \mathbf{Z}_{\varepsilon}^{\Omega}} \int_{\Gamma_{\varepsilon}^{\mathbf{k}}} p_{e} \partial_{t} \mathbf{u}_{\varepsilon} \cdot \mathbf{n}_{\varepsilon}
\end{aligned}\right.
$$

This equality leads us to introduce the energy term, defined for any $t \in[0, T]$ by:

$$
\mathcal{W}_{\varepsilon}(t)=\frac{1}{2} \rho\left\|\partial_{t} \mathbf{u}_{\varepsilon}\right\|_{0, \Omega_{\varepsilon}}^{2}+\frac{1}{2} a_{\varepsilon}\left(\mathbf{u}_{\varepsilon}, \mathbf{u}_{\varepsilon}\right)
$$

Note that, since the Lamé parameters $\lambda, \mu$ are strictly positive, we have

$$
a_{\varepsilon}\left(\mathbf{u}_{\varepsilon}, \mathbf{u}_{\varepsilon}\right)=\int_{\Omega_{\varepsilon}} \lambda\left|\operatorname{div} \mathbf{u}_{\varepsilon}\right|^{2}+2 \mu\left|e\left(\mathbf{u}_{\varepsilon}\right)\right|^{2} \geq 2 \mu\left\|e\left(\mathbf{u}_{\varepsilon}\right)\right\|_{0, \Omega_{\varepsilon}}^{2} .
$$

Hence, thanks to the properties of the extension operator $\widehat{\cdot}$, see Lemma 3.1, and since Korn's inequality holds in the space $\mathbf{V}$, we know that there exists constants $\gamma_{1}, \gamma_{2}>0$ independent of $\varepsilon$ such that

$$
\gamma_{1}\left(\left\|\widehat{\partial_{t} \mathbf{u}_{\varepsilon}}\right\|_{0, \Omega}^{2}+\left|\widehat{\mathbf{u}_{\varepsilon}}\right|_{1, \Omega}^{2}\right) \leq \mathcal{W}_{\varepsilon}(t) \leq \gamma_{2}\left(\left\|\widehat{\partial_{t} \mathbf{u}_{\varepsilon}}\right\|_{0, \Omega}^{2}+\left|\widehat{\mathbf{u}_{\varepsilon}}\right|_{1, \Omega}^{2}\right)
$$

Let $t \in[0, T]$, integrating (3.9) from 0 to $t$ we obtain:

$$
\begin{array}{r}
\mathcal{W}_{\varepsilon}(t)-\mathcal{W}_{\varepsilon}(0)+\mathcal{V}_{\varepsilon}(t)=\int_{0}^{t}\left(\int_{\Omega_{\varepsilon}} \mathbf{f} \cdot \partial_{t} \mathbf{u}_{\varepsilon}+p_{N} \int_{\Gamma_{N}} \partial_{t} \mathbf{u}_{\varepsilon} \cdot \mathbf{n}\right. \\
\left.-p_{e} \sum_{\mathbf{k} \in \mathbf{Z}_{\varepsilon}^{\Omega}} \int_{\Gamma_{\varepsilon}^{\mathbf{k}}} \partial_{t} \mathbf{u}_{\varepsilon} \cdot \mathbf{n}_{\varepsilon}\right) .
\end{array}
$$

Let us evaluate each term in the right-hand side of (3.12). Firstly, we obtain using the Cauchy-Schwartz and Young inequalities:

$$
\int_{0}^{t} \int_{\Omega_{\varepsilon}} \mathbf{f} \cdot \partial_{t} \mathbf{u}_{\varepsilon} \leq \int_{0}^{t} \frac{1}{2}\left\|\widehat{\partial_{t} \mathbf{u}_{\varepsilon}}\right\|_{0, \Omega}^{2}+\int_{0}^{t} \frac{1}{2}\|\mathbf{f}\|_{0, \Omega}^{2}
$$

Secondly, integrating by parts we write:

$$
\int_{0}^{t} \int_{\Gamma_{N}} p_{N} \partial_{t} \mathbf{u}_{\varepsilon} \cdot \mathbf{n}_{\varepsilon}=\left[\int_{\Gamma_{N}} p_{N}(s) \mathbf{u}_{\varepsilon}(s) \cdot \mathbf{n}\right]_{s=0}^{t}-\int_{0}^{t} \int_{\Gamma_{N}} \partial_{t} p_{N} \mathbf{u}_{\varepsilon} \cdot \mathbf{n} .
$$


Using the continuity of the trace operator on $\Gamma_{N}$ in the space $\mathbf{V}$, we obtain:

$\left|\int_{0}^{t} \int_{\Gamma_{N}} p_{N} \partial_{t} \mathbf{u}_{\varepsilon} \cdot \mathbf{n}_{\varepsilon}\right| \leq C\left(\left|p_{N}(t)\right|\left|\widehat{\mathbf{u}_{\varepsilon}}(t)\right|_{1, \Omega}+\left|p_{N}(0)\right|\left|\widehat{\mathbf{u}_{\varepsilon}}(0)\right|_{1, \Omega}+\int_{0}^{t}\left|\partial_{t} p_{N}\right|\left|\widehat{\mathbf{u}_{\varepsilon}}\right|_{1, \Omega}\right)$.

Using Hölder's and Young's inequality, we write for any $\beta>0$ :

$$
\begin{aligned}
\left|\int_{0}^{t} \int_{\Gamma_{N}} p_{N} \partial_{t} \mathbf{u}_{\varepsilon} \cdot \mathbf{n}_{\varepsilon}\right| & \leq \frac{\beta}{2}\left|\widehat{\mathbf{u}_{\varepsilon}(t)}\right|_{1, \Omega}^{2}+\frac{C^{2} \beta^{-1}}{2}\left|p_{N}(t)\right|^{2} \\
& +C\left(\left.\left|p_{N}(0)\right| \widehat{\mid \mathbf{u}_{\varepsilon}}(0)\right|_{1, \Omega}+\frac{1}{2}\left\|\partial_{t} p_{N}\right\|_{L^{2}(0, t)}^{2}+\frac{1}{2} \int_{0}^{t}\left|\widehat{\mathbf{u}_{\varepsilon}}\right|_{1, \Omega}^{2}\right) .
\end{aligned}
$$

Finally, thanks to the initial conditions, we get for any $\beta>0$,

$$
\begin{aligned}
& \left|\int_{0}^{t} \int_{\Gamma_{N}} p_{N} \partial_{t} \mathbf{u}_{\varepsilon} \cdot \mathbf{n}_{\varepsilon}\right| \leq \frac{\beta}{2}\left|\widehat{\mathbf{u}_{\varepsilon}(t)}\right|_{1, \Omega}^{2}+\frac{C}{2} \int_{0}^{t}\left|\widehat{\mathbf{u}_{\varepsilon}}\right|_{1, \Omega}^{2} \\
& \quad+C\left(\left|p_{N}(0)\right|\left|\mathbf{u}_{0}\right|_{1, \Omega}+\frac{1}{2}\left\|\partial_{t} p_{N}\right\|_{L^{2}(0, t)}^{2}+\frac{C \beta^{-1}}{2}\left|p_{N}(t)\right|^{2}\right) .
\end{aligned}
$$

To obtain for the third term an estimate independent of $\varepsilon$ we use the formula:

$$
\sum_{\mathbf{k} \in \mathbf{Z}_{\varepsilon}^{\Omega}} \int_{\Gamma_{\varepsilon}^{\mathbf{k}}} \mathbf{u}_{\varepsilon} \cdot \mathbf{n}_{\varepsilon}=\int_{\Omega \backslash \Omega_{\varepsilon}} \operatorname{div} \widehat{\mathbf{u}_{\varepsilon}}
$$

This leads us to the estimate, valid for all $\beta>0$ :

$$
\begin{aligned}
\left|\int_{0}^{t} p_{e} \sum_{\mathbf{k} \in \mathbf{Z}_{\varepsilon}^{\Omega}} \int_{\Gamma_{\varepsilon}^{\mathbf{k}}} \partial_{t} \mathbf{u}_{\varepsilon} \cdot \mathbf{n}_{\varepsilon}\right| \leq \frac{\beta}{2}\left|\widehat{\mathbf{u}_{\varepsilon}(t)}\right|_{1, \Omega}^{2}+\frac{C}{2} \int_{0}^{t}\left|\widehat{\mathbf{u}_{\varepsilon}}\right|_{1, \Omega}^{2} \\
+C\left(\left|p_{e}(0)\right|\left|\mathbf{u}_{0}\right|_{1, \Omega}+\frac{1}{2}\left\|\partial_{t} p_{e}\right\|_{L^{2}(0, t)}^{2}+\frac{C \beta^{-1}}{2}\left|p_{e}(t)\right|^{2}\right) .
\end{aligned}
$$

Finally, combining (3.12) and all three estimates (3.13), (3.14) and (3.15), we get:

$$
\mathcal{W}_{\varepsilon}(t)+\mathcal{V}_{\varepsilon}(t) \leq \beta\left|\widehat{\mathbf{u}}_{\varepsilon}(t)\right|_{1, \Omega}^{2}+\int_{0}^{t}\left(\frac{1}{2}\left\|\partial_{t} \widehat{\partial \mathbf{u}_{\varepsilon}}\right\|_{0, \Omega}^{2}+C\left|\widehat{\mathbf{u}}_{\varepsilon}\right|_{1, \Omega}^{2}\right)+K,
$$

where $K$ is a constant depending on the initial conditions and the parameters of the problem, but independent of $\varepsilon$ thanks the hypothesis (3.1):

$$
\begin{aligned}
K=\frac{1}{2}\|\mathbf{f}\|_{\mathbf{L}^{2}((0, T) \times \Omega)}^{2}+ & C\left(\left\|p_{N}\right\|_{L^{\infty}(0, T)}+\left\|p_{e}\right\|_{L^{\infty}(0, T)}\right)\left|\mathbf{u}_{0}\right|_{1, \Omega}+\frac{1}{2}\left|p_{N}\right|_{H^{1}(0, T)}^{2} \\
& \left.+\frac{1}{2}\left|p_{e}\right|_{H^{1}(0, T)}^{2}+\frac{C \beta^{-1}}{2}\left(\left\|p_{N}\right\|_{L^{\infty}(0, T)}^{2}+\left\|p_{e}\right\|_{L^{\infty}(0, T)}^{2}\right)\right) \\
& +\gamma_{2}\left(\left\|\mathbf{u}_{0}\right\|_{1, \Omega}^{2}+\left\|\mathbf{u}_{1}\right\|_{0, \Omega}^{2}\right) .
\end{aligned}
$$

Using the bound (3.11), we choose now $\beta=\gamma_{1} / 2$. Gathering all the constants into $C$, we obtain the following estimate, which holds a.e. $t \in(0, T)$ :

$$
\left\|\widehat{\partial}_{t} \mathbf{u}_{\varepsilon}(t)\right\|_{0, \Omega}^{2}+\left|\widehat{\mathbf{u}_{\varepsilon}}(t)\right|_{1, \Omega}^{2}+\mathcal{V}_{\varepsilon}(t) \leq C\left(1+\int_{0}^{t}\left(\left\|\widehat{\partial_{t} \mathbf{u}_{\varepsilon}}\right\|_{0, \Omega}^{2}+\left|\widehat{\mathbf{u}_{\varepsilon}}\right|_{1, \Omega}^{2}\right)\right)
$$


We can then apply Gronwall's Lemma to the real-valued function $t \mapsto$ $\left\|{\widehat{\partial} t \mathbf{u}_{\varepsilon}}_{(}(t)\right\|_{0, \Omega}^{2}+\left|\widehat{\mathbf{u}}_{\varepsilon}(t)\right|_{1, \Omega}^{2}+\mathcal{V}_{\varepsilon}(t)$. We obtain a.e. $t \in(0, T)$,

$$
\left\|\widehat{\partial_{t} \mathbf{u}_{\varepsilon}}(t)\right\|_{0, \Omega}^{2}+\left|\widehat{\mathbf{u}_{\varepsilon}}(t)\right|_{1, \Omega}^{2}+\mathcal{V}_{\varepsilon}(t) \leq C .
$$

\subsection{Two-scale convergence}

To prove the main convergence results of this paper, we use the method of two-scale convergence introduced by G. Nguetseng [31] and then generalized and applied to several important cases by G. Allaire [1], including the case of perforated domains. For the sake of completeness, we recall here some results we will use throughout the Sections 3 and 4 . The basic idea is to formalize an asymptotic expansion such as

$$
u_{\varepsilon}(\mathbf{x})=u(\mathbf{x}, \mathbf{x} / \varepsilon)+\varepsilon u^{1}(\mathbf{x}, \mathbf{x} / \varepsilon)+\varepsilon^{2} u^{2}(\mathbf{x}, \mathbf{x} / \varepsilon)+\ldots
$$

where the functions $u(\mathbf{x}, \mathbf{y}), u^{k}(\mathbf{x}, \mathbf{y})$ are assumed to be $\mathcal{Y}$-periodic in the fast variable $\mathbf{y}$. In this expansion, we can consider $u$ as the macroscopic unknown, while $u^{1}, u^{2}$ are the microscopic correctors. We denote by the subscript "\#" the property of $\mathcal{Y}$-periodicity for functions defined on $\mathcal{Y}$. We recall the definition, extended to account for time dependence (see [30]):

Definition 3.1. Let $\left(u_{\varepsilon}\right)$ be a sequence of functions in $L^{2}((0, T) \times \Omega)$. This sequence is said to two-scale converge to a limit $u \in L^{2}((0, T) \times \Omega \times \mathcal{Y})$ if we have:

$$
\begin{gathered}
\forall \psi(t, \mathbf{x}, \mathbf{y}) \in L^{2}\left((0, T) \times \Omega ; C_{\#}(\mathcal{Y})\right) \\
\lim _{\varepsilon \rightarrow 0} \int_{0}^{T} \int_{\Omega} u_{\varepsilon}(t, \mathbf{x}) \psi\left(t, \mathbf{x}, \frac{\mathbf{x}}{\varepsilon}\right) \mathrm{d} \mathbf{x}=\int_{0}^{T} \int_{\Omega} \int_{Y} u(t, \mathbf{x}, \mathbf{y}) \psi(t, \mathbf{x}, \mathbf{y}) \mathrm{d} \mathbf{y} \mathrm{d} \mathbf{x},
\end{gathered}
$$

and we denote this convergence by a double arrow:

$$
u_{\varepsilon} \rightarrow u \text {. }
$$

The definition extends readily to vector- or tensor-valued functions.

From the analogous results in [1], we extend to time-dependent functions the following compactness result as in [16]:

\section{Lemma 3.2 .}

a) Each bounded sequence in $L^{2}((0, T) \times \Omega)$ contains a subsequence which twoscale converges to a limit $u \in L^{2}((0, T) \times \Omega \times Y)$.

b) Let $\left(u_{\varepsilon}\right)$ be a bounded sequence in $L^{2}\left(0, T ; H^{1}(\Omega)\right)$. Then, there exists $u \in$ $L^{2}\left(0, T ; H^{1}(\Omega)\right)$ and $u^{1} \in L^{2}\left((0, T) \times \Omega ; H_{\#}^{1}(Y) / \mathbb{R}^{d}\right)$ such that up to a subsequence,

$$
u_{\varepsilon} \rightarrow u \text { and } \nabla u_{\varepsilon} \rightarrow \nabla_{\mathbf{x}} u(t, \mathbf{x})+\nabla_{\mathbf{y}} u^{1}(t, \mathbf{x}, \mathbf{y}) .
$$

If in addition $\partial_{t} u_{\varepsilon}$ is bounded in $L^{2}((0, T) \times \Omega)$, then $\partial_{t} u \in L^{2}((0, T) \times \Omega)$ and

$$
\partial_{t} u_{\varepsilon} \rightarrow \partial_{t} u
$$




\subsection{Two-scale convergence procedure}

3.3.1. Convergence of the sequence of solutions

We apply now the two-scale convergence method with the framework described in Section 3.2 to obtain and rigorously justify the homogenized problem as the microscale parameter $\varepsilon$ goes to zero. We focus in particular on the behavior of the functions describing the flow of the air component across the domain, for which the two-scale convergence has not been studied in other papers. In addition to the structure displacement $\mathbf{u}_{\varepsilon}$ solution of the problem (2.4), we will use two new piecewise constant quantities related to the movement of the air and defined as follows:

$$
\begin{array}{r}
q_{\varepsilon}=-\prod_{\varepsilon}\left(\chi_{F, \varepsilon} \operatorname{div} \widehat{\mathbf{u}_{\varepsilon}}\right), \\
\pi_{\varepsilon}=\mathcal{R}_{\varepsilon} q_{\varepsilon} .
\end{array}
$$

The field $q_{\varepsilon}$ describes the local change in volume of the individual air cavities: we have

$$
\int_{\mathcal{Y}_{\varepsilon}^{\mathbf{k}}} q_{\varepsilon}=\varepsilon^{d} q_{\varepsilon}(\mathbf{x})=\int_{\Gamma_{\varepsilon}^{\mathbf{k}}} \mathbf{u}_{\varepsilon} \cdot \mathbf{n}_{\varepsilon} \quad \text { for all } \mathbf{k} \in \mathbf{Z}_{\varepsilon}^{\Omega} \text { and } \mathbf{x} \in \mathcal{Y}_{\varepsilon}^{\mathbf{k}} .
$$

This expression can be compared to (1.12). Remembering (1.13) and the link between $A_{\varepsilon}$ and $\mathcal{R}_{\varepsilon}$ (see Prop. 2.1), $\pi_{\varepsilon}$ is then a primitive with respect to the time variable of the difference between the fluid pressure field and the pressure at the root node. Moreover, the quantity $\mathcal{V}_{\varepsilon}(t)$ writes simply:

$$
\mathcal{V}_{\varepsilon}(t)=\int_{0}^{t} \int_{\Omega}\left(\mathcal{R}_{\varepsilon} \partial_{t} q_{\varepsilon}\right) \partial_{t} q_{\varepsilon}=\int_{0}^{t} \int_{\Omega} \partial_{t} \pi_{\varepsilon} \partial_{t} q_{\varepsilon}
$$

We deduce from the previous energy estimate (3.9) the following result:

Lemma 3.3. The function $q_{\varepsilon}$ belongs to $L^{2}\left(0, T ; L^{2}(\Omega)\right)$, and $\pi_{\varepsilon}$ belongs to $H^{1}\left(0, T ; L^{2}(\Omega)\right)$. Moreover, there exists $C>0$ independent of $\varepsilon$ such that:

$$
\left\|q_{\varepsilon}\right\|_{L^{2}\left(0, T ; L^{2}(\Omega)\right)}+\left\|\mathcal{R}_{\varepsilon} \partial_{t} q_{\varepsilon}\right\|_{L^{2}\left(0, T ; L^{2}(\Omega)\right)} \leq C .
$$

Proof. We obtain the uniform bounds on $q_{\varepsilon}$ in $L^{2}\left(0, T ; L^{2}(\Omega)\right)$ as an immediate consequence of the a priori bounds (3.7). To bound uniformly $\mathcal{R}_{\varepsilon} \partial_{t} q_{\varepsilon}$, we use the Riesz representation in the $L^{2}$ spaces. Let $\psi \in L^{2}((0, T) \times \Omega)$, the Cauchy-Schwartz inequality (2.8) yields:

$$
\begin{aligned}
\left|\int_{0}^{T} \int_{\Omega}\left(\mathcal{R}_{\varepsilon} \partial_{t} q_{\varepsilon}\right) \psi\right|^{2} & \leq\left(\int_{0}^{T} \int_{\Omega}\left(\mathcal{R}_{\varepsilon} \partial_{t} q_{\varepsilon}\right) \partial_{t} q_{\varepsilon}\right)\left(\int_{0}^{T} \int_{\Omega}\left(\mathcal{R}_{\varepsilon} \psi\right) \psi\right) \\
& \leq \mathcal{V}_{\varepsilon}(T)\left\|\mathcal{R}_{\varepsilon}\right\|_{\mathcal{L}\left(L^{2}(\Omega)\right)}\|\psi\|_{L^{2}((0, T) \times \Omega)}^{2} .
\end{aligned}
$$

But we know that $\mathcal{V}_{\varepsilon}(T)$ is uniformly bounded thanks to the a priori bounds (3.7), and that the sequence $\left(\mathcal{R}_{\varepsilon}\right)_{\varepsilon>0}$ converges strongly in $\mathcal{L}\left(L^{2}(\Omega)\right)$, hence it is also uniformly bounded in the operator norm, with respect to $\varepsilon$. 
Therefore, the linear form $\psi \mapsto \int_{0}^{T} \int_{\Omega}\left(\mathcal{R}_{\varepsilon} \partial_{t} q_{\varepsilon}\right) \psi$ is uniformly bounded in $L^{2}((0, T) \times \Omega)^{\prime}$ so $\mathcal{R}_{\varepsilon} \partial_{t} q_{\varepsilon}$ belongs and is uniformly bounded in $L^{2}((0, T) \times \Omega)$, with respect to $\varepsilon$. As a consequence, $\partial_{t} \pi_{\varepsilon}$ is equal to $\mathcal{R}_{\varepsilon} \partial_{t} q_{\varepsilon}$ and $\pi_{\varepsilon}$ belongs to $H^{1}\left(0, T ; L^{2}(\Omega)\right)$.

Then, we have the following result:

Proposition 3.3. Suppose that (3.1) and (2.12) hold. Then, there exists $\mathbf{u} \in$ $L^{2}(0, T ; \mathbf{V})$ with $\partial_{t} \mathbf{u} \in L^{2}(0, T ; \mathbf{X})$ and there exists $\mathbf{u}^{1} \in L^{2}\left((0, T) \times \Omega ; \mathbf{H}_{\#}^{1}(\mathcal{Y}) / \mathbb{R}^{d}\right)$ such that up to a subsequence, still denoted by $\varepsilon$,

$$
\left\{\begin{aligned}
\widehat{\mathbf{u}_{\varepsilon}} & \rightarrow \mathbf{u}, \\
\nabla \widehat{\mathbf{u}_{\varepsilon}} & \rightarrow \nabla_{\mathbf{x}} \mathbf{u}+\nabla_{\mathbf{y}} \mathbf{u}^{1} \\
\widehat{\partial_{t} \mathbf{u}_{\varepsilon}} & \rightarrow \partial_{t} \mathbf{u}
\end{aligned}\right.
$$

Moreover, the function $q_{\varepsilon}$ converges up to a subsequence to a function $q \in$ $L^{2}((0, T) \times \Omega)$ :

$$
q_{\varepsilon} \rightarrow q=-\left((1-\theta) \operatorname{div}_{\mathbf{x}} \mathbf{u}-\int_{\Gamma} \mathbf{u}^{1} \cdot \mathbf{n}^{S}\right), \quad \text { weakly in } L^{2}((0, T) \times \Omega),
$$

where we remind that $\theta=\left|\mathcal{Y}_{S}\right|$ is the proportion of structure in the material, and the function $\pi_{\varepsilon}$ converges up to a subsequence to $\pi \in H^{1}\left(0, T ; L^{2}(\Omega)\right)$ :

$$
\begin{array}{cr}
\pi_{\varepsilon}=\mathcal{R}_{\varepsilon} q_{\varepsilon} \rightarrow \pi=\mathcal{R} q & \text { strongly in } L^{2}((0, T) \times \Omega), \\
\partial_{t} \pi_{\varepsilon}=\mathcal{R}_{\varepsilon} \partial_{t} q_{\varepsilon} \rightarrow \partial_{t} \pi=\partial_{t}(\mathcal{R} q) & \text { weakly in } L^{2}((0, T) \times \Omega) .
\end{array}
$$

Proof. Thanks to the a priori bounds (3.7), we can apply Lemma 3.2. We deduce that (3.21) holds. Moreover, due to this two-scale convergence of $\mathbf{u}_{\varepsilon}(3.21)$, we have the following weak convergence result:

$$
\chi_{F, \varepsilon} \operatorname{div} \widehat{\mathbf{u}_{\varepsilon}} \rightarrow \int_{\mathcal{Y}_{F}}\left(\operatorname{div}_{\mathbf{x}} \mathbf{u}+\operatorname{div}_{\mathbf{y}} \mathbf{u}^{1}\right), \quad \text { weakly in } L^{2}((0, T) \times \Omega) .
$$

It is then easy to verify that

$q_{\varepsilon} \rightarrow \int_{\mathcal{Y}_{F}}\left(\operatorname{div}_{\mathbf{x}} \mathbf{u}+\operatorname{div}_{\mathbf{y}} \mathbf{u}^{1}\right)=\left|\mathcal{Y}_{F}\right| \operatorname{div}_{\mathbf{x}} \mathbf{u}-\int_{\Gamma} \mathbf{u}^{1} \cdot \mathbf{n}^{S}, \quad$ weakly in $L^{2}((0, T) \times \Omega)$.

Now, let us write

$$
\mathcal{R}_{\varepsilon} q_{\varepsilon}-\mathcal{R} q=\left(\mathcal{R}_{\varepsilon}-\mathcal{R}\right) q_{\varepsilon}+\mathcal{R}\left(q_{\varepsilon}-q\right)
$$

Thanks to the compactness of $\mathcal{R}$ (see Remark 2.3), we deduce that, up to a subsequence, $\mathcal{R}\left(q_{\varepsilon}-q\right)$ converges strongly to zero in $L^{2}((0, T) \times \Omega)$. Moreover, the uniform bounds on $q_{\varepsilon}$ (3.20) combined with the strong convergence of the sequence $\left(\mathcal{R}_{\varepsilon}\right)_{\varepsilon>0}$ imply that $\left(\mathcal{R}_{\varepsilon}-\mathcal{R}\right) q_{\varepsilon}$ converges strongly to zero in $L^{2}((0, T) \times \Omega)$. Thus, we obtain the desired result:

$$
\mathcal{R}_{\varepsilon} q_{\varepsilon} \rightarrow \mathcal{R} q \quad \text { strongly in } L^{2}((0, T) \times \Omega) .
$$


As a consequence,

$$
\mathcal{R}_{\varepsilon} \partial_{t} q_{\varepsilon} \rightarrow \partial_{t}(\mathcal{R} q) \quad \text { in } \mathcal{D}^{\prime}\left(0, T ; L^{2}(\Omega)\right) .
$$

We know thanks to (3.20) that the sequence of pressure fields $\left(\mathcal{R}_{\varepsilon} \partial_{t} q_{\varepsilon}\right)$ is uniformly bounded in $L^{2}((0, T) \times \Omega)$, so it converges weakly up to a subsequence in that space. Combined with $(3.25)$, we can identify this weak limit with $\partial_{t}(\mathcal{R} q)$ so we have obtained:

$$
\mathcal{R} q \in H^{1}\left(0, T ; L^{2}(\Omega)\right) \text { and } \mathcal{R}_{\varepsilon} \partial_{t} q_{\varepsilon} \rightarrow \partial_{t}(\mathcal{R} q) \quad \text { weakly in } L^{2}((0, T) \times \Omega) .
$$

\subsubsection{The two-scale limit problem: main result}

Define the Hilbert space $\left.\mathbf{H}=L^{2}\left(\Omega ; \mathbf{H}_{\#}^{1}\left(\mathcal{Y}_{S}\right) / \mathbb{R}^{d}\right)\right)$. We are now in a position to show how the two-scale convergence process sketched in Prop. 3.3 gives us an effective macroscopic model of the parenchyma mechanical behavior. First, let us write the two-scale problem obtained by taking the two-scale limit term by term in the variational formulation (3.2). Let $\phi$ be a test function in $\mathcal{D}([0, T)$ )and $\mathbf{v}$ in $\mathbf{V} \cap$ $C^{\infty}(\bar{\Omega}), \mathbf{v}^{1}$ in $\mathcal{D}\left(\Omega ; C_{\#}^{\infty}(\mathcal{Y})\right)$. We will denote the time derivative of $\phi$ by $\phi^{\prime}$ to simplify notations.

As is standard when using two-scale convergence, we choose the test function $\mathbf{v}_{\varepsilon} \in \mathbf{V}_{\varepsilon}$ in the variational formulation (3.2) defined by:

$$
\mathbf{v}_{\varepsilon}(\mathbf{x})=\mathbf{v}(\mathbf{x})+\varepsilon \mathbf{v}^{1}\left(\mathbf{x}, \frac{\mathbf{x}}{\varepsilon}\right) \text {. }
$$

We integrate in time against $\phi$ and we use the extension operator $\hat{\cdot}$ to write all integrals over $\Omega_{\varepsilon}$ in variational formulation (3.2) as integrals over $\Omega$. Since $\phi$ has compact support in $[0, T)$ :

$$
\left\{\begin{aligned}
-\int_{0}^{T} \phi^{\prime} & \int_{\Omega} \rho \partial_{t} \widehat{\mathbf{u}_{\varepsilon}} \cdot \mathbf{v}_{\varepsilon} \chi_{S, \varepsilon} \mathrm{d} \mathbf{x} \mathrm{d} t \\
& -\phi(0) \int_{\Omega} \rho \mathbf{u}_{1} \cdot \mathbf{v}_{\varepsilon} \chi_{S, \varepsilon} \mathrm{d} \mathbf{x} \\
& +\int_{0}^{T} \phi \int_{\Omega} \lambda \operatorname{div} \widehat{\mathbf{u}_{\varepsilon}} \operatorname{div}\left(\mathbf{v}_{\varepsilon}\right) \chi_{S, \varepsilon}+2 \mu e\left(\widehat{\mathbf{u}_{\varepsilon}}\right): e\left(\mathbf{v}_{\varepsilon}\right) \chi_{S, \varepsilon} \mathrm{d} \mathbf{x} \mathrm{d} t \\
& -\int_{0}^{T} \phi^{\prime} \int_{\Omega} \mathcal{R}_{\varepsilon}\left(\chi_{F, \varepsilon} \operatorname{div} \widehat{\mathbf{u}_{\varepsilon}}\right) \Pi_{\varepsilon}\left(\chi_{F, \varepsilon} \operatorname{div} \widehat{\mathbf{v}_{\varepsilon}}\right) \mathrm{d} \mathbf{x} \mathrm{d} t \\
& -\phi(0) \int_{\Omega} \mathcal{R}_{\varepsilon}\left(\chi_{F, \varepsilon} \operatorname{div} \mathbf{u}_{0}\right) \Pi_{\varepsilon}\left(\chi_{F, \varepsilon} \operatorname{div} \widehat{\mathbf{v}_{\varepsilon}}\right) \mathrm{d} \mathbf{x} \\
& =\int_{0}^{T} \phi \int_{\Omega} \mathbf{f} \cdot \mathbf{v}_{\varepsilon} \chi_{S, \varepsilon} \mathrm{d} \mathbf{x} \mathrm{d} t \\
& +\int_{0}^{T} \phi \int_{\Gamma_{N}} p_{N} \mathbf{v}_{\varepsilon} \cdot \mathbf{n} \mathrm{d} t \\
& -\int_{0}^{T} \phi \int_{\Gamma_{\varepsilon}} p_{e} \mathbf{v}_{\varepsilon} \cdot \mathbf{n}_{\varepsilon} \mathrm{d} t .
\end{aligned}\right.
$$


Using the definition of two-scale convergence (3.16) and the convergences obtained in Proposition 3.3, it is standard to pass to the limit in the first three terms in the left-hand side and in the first two terms in the right-hand side of (3.26). Note that to compute the limits the indicator function $\chi_{S, \varepsilon}$ of the structure domain is associated with the test function.

We now focus our analysis on the remaining, non-standard terms. Using the function $q_{\varepsilon}$ introduced in Prop. 3.3, we have:

$$
\int_{\Omega} \mathcal{R}_{\varepsilon}\left(\chi_{F, \varepsilon} \operatorname{div} \widehat{\mathbf{u}_{\varepsilon}}\right) \Pi_{\varepsilon}\left(\chi_{F, \varepsilon} \operatorname{div} \widehat{\mathbf{v}_{\varepsilon}}\right) \mathrm{d} \mathbf{x}=\int_{\Omega} \mathcal{R}_{\varepsilon} q_{\varepsilon} \Pi_{\varepsilon}\left(\chi_{F, \varepsilon} \operatorname{div} \widehat{\mathbf{v}_{\varepsilon}}\right) \mathrm{d} \mathbf{x} .
$$

We recall the following technical Lemma (see e.g. [3]):

Lemma 3.4. Let $\Pi_{\varepsilon}$ be the projection operator defined in Definition 2.1. Then, for all $\phi \in \mathcal{D}\left(\Omega, C_{\#}^{\infty}(\mathcal{Y})\right)$,

$$
\begin{aligned}
\Pi_{\varepsilon}\left(\phi\left(\cdot, \frac{\cdot}{\varepsilon}\right)\right) & \rightarrow \int_{\mathcal{Y}} \phi(\cdot, \mathbf{y}) \mathrm{d} \mathbf{y}, \quad L^{2}(\Omega) \text {-strongly }, \\
\Pi_{\varepsilon}\left(\chi_{F, \varepsilon} \phi\left(\cdot, \frac{\dot{\varepsilon}}{\varepsilon}\right)\right) & \rightarrow \int_{\mathcal{Y}_{F}} \phi(\cdot, \mathbf{y}) \mathrm{d} \mathbf{y} \quad L^{2}(\Omega) \text {-strongly. }
\end{aligned}
$$

Then thanks to the convergence of $\pi_{\varepsilon}$ presented in Prop. 3.3 we have:

$$
\begin{aligned}
\int_{0}^{T} \phi^{\prime} \int_{\Omega} \pi_{\varepsilon} \Pi_{\varepsilon}\left(\chi_{F, \varepsilon} \operatorname{div} \widehat{\mathbf{v}_{\varepsilon}}\right) \mathrm{d} \mathbf{x} \mathrm{d} t \rightarrow \\
\quad \int_{0}^{T} \phi^{\prime} \int_{\Omega} \pi\left((1-\theta) \operatorname{div}_{\mathbf{x}} \mathbf{v}-\int_{\Gamma} \mathbf{v}^{1} \cdot \mathbf{n}^{S}\right) \mathrm{d} \mathbf{x} \mathrm{d} t .
\end{aligned}
$$

Next, since $\mathcal{R}$ is a compact operator,

$$
\mathcal{R}_{\varepsilon}\left(\chi_{F, \varepsilon} \operatorname{div} \mathbf{u}_{0}\right) \rightarrow \mathcal{R}\left((1-\theta) \operatorname{div}_{\mathbf{x}} \mathbf{u}_{0}\right) \text { strongly in } L^{2}(\Omega),
$$

SO

$$
\begin{aligned}
\phi(0) \int_{\Omega} \mathcal{R}_{\varepsilon}\left(\chi_{F, \varepsilon} \operatorname{div} \mathbf{u}_{0}\right) \Pi_{\varepsilon}\left(\chi_{F, \varepsilon} \operatorname{div} \widehat{\mathbf{v}_{\varepsilon}}\right) \mathrm{d} \mathbf{x} \mathrm{d} t \rightarrow \\
\quad \phi(0) \int_{\Omega}(1-\theta) \mathcal{R}\left(\operatorname{div}_{\mathbf{x}} \mathbf{u}_{0}\right)\left((1-\theta) \operatorname{div}_{\mathbf{x}} \mathbf{v}-\int_{\Gamma} \mathbf{v}^{1} \cdot \mathbf{n}^{S}\right) \mathrm{d} \mathbf{x} \mathrm{d} t .
\end{aligned}
$$

Similarly we pass to the limit in the last term of (3.26). We rewrite the boundary integral using the divergence theorem:

$$
\int_{\Gamma_{\varepsilon}} p_{e} \mathbf{v}_{\varepsilon} \cdot \mathbf{n}_{\varepsilon} \mathrm{d} \mathbf{x}=p_{e} \int_{\mathcal{Y}_{F, \varepsilon}} \operatorname{div}\left(\mathbf{v}_{\varepsilon}\right) \mathrm{d} \mathbf{x}=p_{e} \int_{\Omega} \operatorname{div}\left(\mathbf{v}_{\varepsilon}\right) \chi_{F, \varepsilon} \mathrm{d} \mathbf{x} .
$$

Hence:

$$
\int_{0}^{T} \phi \int_{\Gamma_{\varepsilon}} p_{e} \mathbf{v}_{\varepsilon} \cdot \mathbf{n}_{\varepsilon} \mathrm{d} \mathbf{x} \mathrm{d} t \rightarrow \int_{0}^{T} \phi \int_{\Omega} p_{e}\left((1-\theta) \operatorname{div}_{\mathbf{x}} \mathbf{v}-\int_{\Gamma} \mathbf{v}^{1} \cdot \mathbf{n}^{S}\right) \mathrm{d} \mathbf{x} \mathrm{d} t
$$


Thus $\mathbf{u}$ and $\mathbf{u}^{1}$ satisfy:

$$
\left\{\begin{aligned}
-\int_{0}^{T} \phi^{\prime}(\theta \rho \mathbf{u}, \mathbf{v})_{0, \Omega}-\phi(0)\left(\theta \rho \mathbf{u}_{1}, \mathbf{v}\right)_{0, \Omega} \\
\quad-\int_{0}^{T} \phi^{\prime} r_{\#}\left(\left(\mathbf{u}, \mathbf{u}^{1}\right),\left(\mathbf{v}, \mathbf{v}^{1}\right)\right)-\phi(0) r_{\#}\left(\left(\mathbf{u}_{0}, 0\right),\left(\mathbf{v}, \mathbf{v}^{1}\right)\right) \\
\quad+\int_{0}^{T} \phi a_{\#}\left(\left(\mathbf{u}, \mathbf{u}^{1}\right),\left(\mathbf{v}, \mathbf{v}^{1}\right)\right)=\int_{0}^{T} \phi \ell_{\#}\left(\mathbf{v}, \mathbf{v}^{1}\right)
\end{aligned}\right.
$$

where we have introduced the two bilinear forms $a_{\#}, r_{\#}$ and the linear form $\ell_{\#}$ on $\mathbf{V} \times \mathbf{H}$ defined as:

$$
\begin{aligned}
a_{\#}\left(\left(\mathbf{u}, \mathbf{u}^{1}\right),\left(\mathbf{v}, \mathbf{v}^{1}\right)\right)= & \int_{\Omega} \int_{\mathcal{Y}_{S}} \lambda\left(\operatorname{div}_{\mathbf{x}} \mathbf{u}+\operatorname{div}_{\mathbf{y}} \mathbf{u}^{1}\right)\left(\operatorname{div}_{\mathbf{x}} \mathbf{v}+\operatorname{div}_{\mathbf{y}} \mathbf{v}^{1}\right) \\
& +2 \mu\left(e_{\mathbf{x}}(\mathbf{u})+e_{\mathbf{y}}\left(\mathbf{u}^{1}\right)\right):\left(e_{\mathbf{x}}(\mathbf{v})+e_{\mathbf{y}}\left(\mathbf{v}^{1}\right)\right), \\
r_{\#}\left(\left(\mathbf{u}, \mathbf{u}^{1}\right),\left(\mathbf{v}, \mathbf{v}^{1}\right)\right)= & \int_{\Omega} \mathcal{R}\left((1-\theta) \operatorname{div}_{\mathbf{x}} \mathbf{u}-\int_{\Gamma} \mathbf{u}^{1} \cdot \mathbf{n}^{S}\right) \\
& \cdot\left((1-\theta) \operatorname{div}_{\mathbf{x}} \mathbf{v}-\int_{\Gamma} \mathbf{v}^{1} \cdot \mathbf{n}^{S}\right), \\
\ell_{\#}\left(\mathbf{v}, \mathbf{v}^{1}\right)= & \int_{\Omega} \theta \mathbf{f} \cdot \mathbf{v}+\int_{\Gamma_{N}} p_{N} \mathbf{v} \cdot \mathbf{n} \\
& -\int_{\Omega} p_{e}\left((1-\theta) \operatorname{div}_{\mathbf{x}} \mathbf{v}-\int_{\Gamma} \mathbf{v}^{1} \cdot \mathbf{n}^{S}\right) .
\end{aligned}
$$

Since $\mathbf{u}$ and $\mathbf{u}^{1}$ satisfy the equation (3.30) for all test functions $\phi \in \mathcal{D}([0, T)$ ), we are now in a position to show that they are the solution of a well-posed two-scale problem, which is as a first step identified as follow.

Initial conditions. Thanks to the regularity of $\mathbf{u}$ and $\pi$ presented in Prop. 3.3 we can identify some initial conditions satisfied by the limits $\mathbf{u}$ and $\mathbf{u}^{1}$ from the limit system 3.30. Using the Stokes formula, we identify

$$
\partial_{t} \mathbf{u}(0)=\mathbf{u}^{1}
$$

and also

$$
\pi(0)=\left.\mathcal{R}\left((1-\theta) \operatorname{div}_{\mathbf{x}} \mathbf{u}-\int_{\Gamma} \mathbf{u}^{1} \cdot \mathbf{n}^{S}\right)\right|_{t=0}=\mathcal{R}\left((1-\theta) \operatorname{div}_{\mathbf{x}} \mathbf{u}_{0}\right) .
$$

In addition, we know also that $W^{1, \infty}(0, T ; \mathbf{X}) \cap L^{\infty}(0, T ; \mathbf{V})$ is compactly imbedded in $C([0, T] ; \mathbf{X})$. Therefore, since $\left(\widehat{\mathbf{u}_{\varepsilon}}\right)_{\varepsilon>0}$ is a bounded sequence in $W^{1, \infty}(0, T ; \mathbf{X}) \cap$ $L^{\infty}(0, T ; \mathbf{V})$, up to a subsequence we have the strong convergence of $\widehat{\mathbf{u}_{\varepsilon}}$ to $\mathbf{u}$ in $C(0, T ; \mathbf{X})$. As a consequence, $\widehat{\mathbf{u}_{\varepsilon}}(0)$ converges strongly to $\mathbf{u}(0)$ in $\mathbf{X}$. Moreover, we know that:

$$
\widehat{\mathbf{u}_{\varepsilon}}(0) \chi_{S, \varepsilon}=\mathbf{u}_{0} \chi_{S, \varepsilon},
$$


so by taking the two-scale limit in both sides of the latter identity, we obtain

$$
\mathbf{u}(0) \chi_{S}=\mathbf{u}_{0} \chi_{S} \text { in } \Omega \times \mathcal{Y} .
$$

We obtain thus the initial condition:

$$
\mathbf{u}(0)=\mathbf{u}_{0} \text { a.e. in } \Omega .
$$

Notice that the latter identity results in an additional initial condition for the twoscale limits $\mathbf{u}$ and $\mathbf{u}^{1}$ compared to the system (3.2).

Two-scale problem. Using arbitrary test functions $\phi$ in $\mathcal{D}(0, T) \subset H_{0}^{1}(0, T)$, the following result holds by a density argument in identity (3.30) and thanks to the regularity in the time variable of the unknowns obtained in Prop. 3.3:

Proposition 3.4. The limits $\mathbf{u}, \mathbf{u}_{1}$ are solutions of the two-scale variational problem: for all $\mathbf{v} \in \mathbf{V}, \mathbf{v}^{1} \in \mathbf{H}$,

$$
\left\{\begin{aligned}
\frac{\mathrm{d}}{\mathrm{d} t}\left(\theta \rho \partial_{t} \mathbf{u}, \mathbf{v}\right)_{0, \Omega} & +\frac{\mathrm{d}}{\mathrm{d} t} r_{\#}\left(\left(\mathbf{u}, \mathbf{u}^{1}\right),\left(\mathbf{v}, \mathbf{v}^{1}\right)\right) \\
& +a_{\#}\left(\left(\mathbf{u}, \mathbf{u}^{1}\right),\left(\mathbf{v}, \mathbf{v}^{1}\right)\right)=\ell_{\#}\left(\mathbf{v}, \mathbf{v}^{1}\right)
\end{aligned} \quad \text { in } H^{-1}(0, T),\right.
$$

with the initial conditions

$$
\left\{\begin{array}{l}
\mathbf{u}(0)=\mathbf{u}_{0}, \partial_{t} \mathbf{u}(0)=\mathbf{u}^{1}, \\
\pi(0)=\left.\mathcal{R}\left((1-\theta) \operatorname{div}_{\mathbf{x}} \mathbf{u}-\int_{\Gamma} \mathbf{u}^{1} \cdot \mathbf{n}^{S}\right)\right|_{t=0}=\mathcal{R}\left((1-\theta) \operatorname{div}_{\mathbf{x}} \mathbf{u}_{0}\right)
\end{array}\right.
$$

We have the following result:

Proposition 3.5. The variational problem (3.34) has a unique solution in the space of pairs $\left(\mathbf{u}, \mathbf{u}^{1}\right)$ such that

$$
\left\{\begin{array}{l}
\left.\mathbf{u} \in L^{2}(0, T ; \mathbf{V}) \cap H^{1}(0, T) ; \mathbf{X}\right) \\
\mathbf{u}^{1} \in L^{2}(0, T ; \mathbf{H}) \\
\pi=\mathcal{R}\left((1-\theta) \operatorname{div}_{\mathbf{x}} \mathbf{u}-\int_{\Gamma} \mathbf{u}^{1} \cdot \mathbf{n}^{S}\right) \in H^{1}\left(0, T ; L^{2}(\Omega)\right) .
\end{array}\right.
$$

Proof. Let $\left(\mathbf{u}_{1}, \mathbf{u}_{1}^{1}\right)$ and $\left(\mathbf{u}_{2}, \mathbf{u}_{2}^{1}\right)$ be two solutions of $(3.34)$ and let $\left(\mathbf{w}, \mathbf{w}^{1}\right)=$ $\left(\mathbf{u}_{1}-\mathbf{u}_{2}, \mathbf{u}_{1}^{1}-\mathbf{u}_{2}^{2}\right)$. Let $s \in[0, T)$. We introduce the following test functions, following a classical idea (see [25]):

$$
\mathbf{v}=\left\{\begin{array}{ll}
-\int_{t}^{s} \mathbf{w} & 0 \leq t \leq s \\
0 & t>s
\end{array}, \quad \mathbf{v}^{1}=\left\{\begin{array}{ll}
-\int_{t}^{s} \mathbf{w}^{1} & 0 \leq t \leq s \\
0 & t>s
\end{array} .\right.\right.
$$

Then we have, using (3.34a) with zero right-hand side,

$$
\begin{aligned}
\int_{0}^{T}\left\langle\theta \rho \partial_{t t} \mathbf{w}, \mathbf{v}\right\rangle+a_{\#}\left(\left(\mathbf{w}, \mathbf{w}^{1}\right),\left(\mathbf{v}, \mathbf{v}^{1}\right)\right) \\
\quad+\int_{0}^{T} \int_{\Omega} \frac{\partial}{\partial t} \mathcal{R}\left((1-\theta) \operatorname{div}_{\mathbf{x}} \mathbf{w}-\int_{\Gamma} \mathbf{w}^{1} \cdot \mathbf{n}^{S}\right)\left((1-\theta) \operatorname{div}_{\mathbf{x}} \mathbf{v}-\int_{\Gamma} \mathbf{v}^{1} \cdot \mathbf{n}^{S}\right)=0,
\end{aligned}
$$


where $\langle\cdot, \cdot\rangle$ is the duality pairing between $\mathbf{V}^{\prime}$ and $\mathbf{V}$. Integrating by parts in time, we obtain:

$$
\begin{aligned}
\int_{0}^{T} & -\left(\theta \rho \partial_{t} \mathbf{w}, \partial_{t} \mathbf{v}\right)_{0, \Omega}+a_{\#}\left(\left(\mathbf{w}, \mathbf{w}^{1}\right),\left(\mathbf{v}, \mathbf{v}^{1}\right)\right) \\
& -\int_{0}^{T} \int_{\Omega} \mathcal{R}\left((1-\theta) \operatorname{div}_{\mathbf{x}} \mathbf{w}-\int_{\Gamma} \mathbf{w}^{1} \cdot \mathbf{n}^{S}\right) \frac{\partial}{\partial t}\left((1-\theta) \operatorname{div}_{\mathbf{x}} \mathbf{v}-\int_{\Gamma} \mathbf{v}^{1} \cdot \mathbf{n}^{S}\right)=0,
\end{aligned}
$$

where we have used the fact that

$$
\partial_{t} \mathbf{w}(0)=\mathbf{u}_{1}-\mathbf{u}_{1}=\mathbf{0}, \quad \mathbf{v}(T)=\mathbf{0}, \quad \mathbf{v}^{1}(T)=\mathbf{0},
$$

and also

$$
\left.\mathcal{R}\left((1-\theta) \operatorname{div}_{\mathbf{x}} \mathbf{w}-\int_{\Gamma} \mathbf{w}^{1} \cdot \mathbf{n}^{S}\right)\right|_{t=0}=\mathcal{R}\left((1-\theta) \operatorname{div}_{\mathbf{x}}\left(\mathbf{u}_{0}-\mathbf{u}_{0}\right)\right)=0 .
$$

Since $\mathcal{R}$ is a positive operator and $\partial_{t} \mathbf{v}=\mathbf{w}, \partial_{t} \mathbf{v}^{1}=\mathbf{w}^{1}$, this yields:

$$
\int_{0}^{s} \frac{1}{2} \frac{\mathrm{d}}{\mathrm{d} t}\left(-\theta \rho\|\mathbf{w}\|_{0, \Omega}^{2}+a_{\#}\left(\left(\mathbf{v}, \mathbf{v}^{1}\right),\left(\mathbf{v}, \mathbf{v}^{1}\right)\right)\right) \geq 0 .
$$

Hence, because $\mathbf{w}(0)=\mathbf{u}_{0}-\mathbf{u}_{0}=\mathbf{0}$ :

$$
-\theta \rho\|\mathbf{w}(s)\|_{0, \Omega}^{2}-a_{\#}\left(\left(\mathbf{v}(0), \mathbf{v}^{1}(0)\right),\left(\mathbf{v}(0), \mathbf{v}^{1}(0)\right)\right) \geq 0 .
$$

Since $a_{\#}$ is positive, we obtain $\mathbf{w}(s)=\mathbf{0}$ for all $s \in(0, T)$. We conclude that the solution of (3.34) is unique.

The following Theorem is the main result of this section and is obtained as an immediate consequence of the previous results:

Theorem 3.1. (Asymptotic two-scale formulation)

Suppose conditions (2.12) and (3.1) hold. Let $\left(\mathbf{u}_{\varepsilon}\right)_{\varepsilon>0}$ be the sequence of solutions of the family of problems (2.4) when $\varepsilon$ varies.

Then the three sequences $\left(\widehat{\mathbf{u}_{\varepsilon}} \chi_{S, \varepsilon}\right)_{\varepsilon>0},\left(\widehat{\partial}_{t} \mathbf{u}_{\varepsilon} \chi_{S, \varepsilon}\right)_{\varepsilon>0}$ and $\left(\nabla\left(\widehat{\mathbf{u}_{\varepsilon}}\right) \chi_{S, \varepsilon}\right)_{\varepsilon>0}$ twoscale converge respectively to $\mathbf{u} \chi_{S}, \partial_{t} \mathbf{u} \chi_{S}$ and $\left(\nabla_{\mathbf{x}} \mathbf{u}+\nabla_{\mathbf{y}} \mathbf{u}^{1}\right) \chi_{S}$ in $L^{2}((0, T) \times$ $\Omega \times \mathcal{Y})$, where $\left(\mathbf{u},\left.\mathbf{u}^{1}\right|_{\Omega \times \mathcal{Y}_{S}}\right)$ is the unique solution of the two-scale variational problem (3.34).

\subsection{Cell problems, correctors and the homogenized problem}

In this part, we are going to eliminate the microscopic additional unknown $\mathbf{u}^{1}$ from the two-scale variational problem (3.34). This is a standard step to exhibit the macroscopic properties (memory effects, effective elastic tensor...) of the homogenized material associated with the homogenized problem. For simplicity, we will consider from now on that $\mathbf{u}^{1}$ is defined on $\Omega \times \mathcal{Y}_{S}$ only. 
Cell problem Taking the test function $\mathbf{v}$ to be 0 in Eq. (3.34), we obtain a nonstandard cell problem:

$$
\left\{\begin{array}{l}
\text { For all } \mathbf{v}^{1} \in \mathbf{H}, \quad \text { a.e. } t \in(0, T) \\
\int_{\Omega} \int_{\mathcal{Y}_{S}}\left(\operatorname{div}_{\mathbf{y}}\left(\mathbf{u}^{1}\right) \operatorname{div}_{\mathbf{y}}\left(\mathbf{v}^{1}\right)+2 \mu e_{\mathbf{y}}\left(\mathbf{u}^{1}\right): e_{\mathbf{y}}\left(\mathbf{v}^{1}\right)\right) \\
-\int_{\Omega} \frac{\partial}{\partial t} \mathcal{R}\left((1-\theta) \operatorname{div}_{\mathbf{x}} \mathbf{u}-\int_{\Gamma} \mathbf{u}^{1} \cdot \mathbf{n}_{S}\right) \int_{\Gamma} \mathbf{v}^{1} \cdot \mathbf{n}^{S} \\
=-\int_{\Omega} \int_{\mathcal{Y}_{S}}\left(\lambda \operatorname{div}_{\mathbf{x}}(\mathbf{u}) \operatorname{div}_{\mathbf{y}}\left(\mathbf{v}^{1}\right)+2 \mu e_{\mathbf{x}}(\mathbf{u}): e_{\mathbf{y}}\left(\mathbf{v}^{1}\right)\right)-\int_{\Omega} p_{e} \int_{\Gamma} \mathbf{v}^{1} \cdot \mathbf{n}^{S} .
\end{array}\right.
$$

Note that all the cell problems are coupled across $\Omega$ by the non-local operator $\mathcal{R}$ which acts on the corrector $\mathbf{u}^{1}$. In order to decouple the cell problems, we need to use $\pi$ as an additional macroscopic unknown in the system. Then this problem can be transformed into a family of problems parameterized by the $\mathbf{x}$ variable by a density argument, using test functions of the form $\mathbf{w}^{1}(\mathbf{x}, \mathbf{y})=\phi(\mathbf{x}) \mathbf{v}^{1}(\mathbf{y})$ :

$$
\left\{\begin{aligned}
& \text { For all } \mathbf{v}^{1} \in \mathbf{H}_{\#}^{1}\left(\mathcal{Y}_{S}\right) / \mathbb{R}^{d}, \quad \text { a.e. }(t, \mathbf{x}) \in(0, T) \times \Omega \\
\int_{\mathcal{Y}_{S}} & \left(\lambda \operatorname{div}_{\mathbf{y}}\left(\mathbf{u}^{1}\right) \operatorname{div}_{\mathbf{y}}\left(\mathbf{v}^{1}\right)+2 \mu e_{\mathbf{y}}\left(\mathbf{u}^{1}\right): e_{\mathbf{y}}\left(\mathbf{v}^{1}\right)\right) \\
= & \left(\partial_{t} \pi-p_{e}\right)\left(\int_{\Gamma} \mathbf{v}^{1} \cdot \mathbf{n}^{S}\right) \\
& -\left(\lambda \operatorname{div}_{\mathbf{x}}(\mathbf{u}) \operatorname{Id}+2 \mu e_{\mathbf{x}}(\mathbf{u})\right):\left(\int_{\mathcal{Y}_{S}} e_{\mathbf{y}}\left(\mathbf{v}^{1}\right)\right)
\end{aligned}\right.
$$

This is now a linear elliptic problem, parameterized by $e_{\mathbf{x}}(\mathbf{u})$ and $\partial_{t} \pi-p_{e}$. Also $\pi$ depends itself on $\mathbf{u}^{1}$, see (3.24):

$$
\pi=-\mathcal{R}\left((1-\theta) \operatorname{div}_{\mathbf{x}} \mathbf{u}-\int_{\Gamma} \mathbf{u}^{1} \cdot \mathbf{n}^{S}\right) .
$$

Thus, this introduction of the unknown $\pi$ in the problem will give us a coupled system with unknowns $\left(\pi, \mathbf{u}^{1}\right)$ where the cell problems (3.36) are local. As is standard, we are going to take advantage of the superposition principle by introducing the so-called correctors. We define the auxiliary functions $\mathbf{p}^{k l} \in \mathbf{H}^{1}\left(Y_{S}\right)$ by:

$$
\mathbf{p}^{k l}(\mathbf{y})=\frac{1}{2}\left(y_{k} \mathbf{e}^{l}+y_{l} \mathbf{e}^{k}\right) \quad \text { for } 1 \leq k, l \leq d,
$$

where the vectors $\mathbf{e}^{k}$ for $1 \leq k \leq d$ are the unit vectors of $\mathbb{R}^{d}$ whose components are $\mathbf{e}_{l}^{k}=\delta_{k l}$ for $1 \leq k, l \leq d$. We recall that we use the Einstein convention for summing here. In view of (3.36), we can decompose the solution $\mathbf{u}^{1}$ using the superposition principle:

$$
\mathbf{u}^{1}=e_{\mathbf{x}}(\mathbf{u})_{k l} \chi^{k l}+\left(\partial_{t} \pi-p_{e}\right) \chi^{0}
$$


where the functions $\chi^{k l}$ are correctors in $\mathbf{H}_{\#}^{1}\left(\mathcal{Y}_{S}\right) / \mathbb{R}^{d}$, solution of the variational cell problems:

$$
\left\{\begin{array}{l}
\text { For all } \mathbf{v}^{1} \in \mathbf{H}_{\#}^{1}\left(\mathcal{Y}_{S}\right) / \mathbb{R}^{d}, \\
\int_{\mathcal{Y}_{S}}\left(\lambda \operatorname{div}_{\mathbf{y}}\left(\chi^{k l}\right) \operatorname{Id}+2 \mu e_{\mathbf{y}}\left(\chi^{k l}\right)\right): e_{\mathbf{y}}\left(\mathbf{v}^{1}\right) \\
\quad=-\int_{\mathcal{Y}_{S}}\left(\lambda \operatorname{div}_{\mathbf{y}}\left(\mathbf{p}^{k l}\right) \operatorname{Id}+2 \mu e_{\mathbf{y}}\left(\mathbf{p}^{k l}\right)\right): e_{\mathbf{y}}\left(\mathbf{v}^{1}\right),
\end{array}\right.
$$

and the corrector $\chi^{0}$ in $\mathbf{H}_{\#}^{1}\left(\mathcal{Y}_{S}\right) / \mathbb{R}^{d}$ is associated with the variational cell problem:

$$
\left\{\begin{array}{l}
\text { For all } \mathbf{v}^{1} \in \mathbf{H}_{\#}^{1}\left(\mathcal{Y}_{S}\right) / \mathbb{R}^{d}, \\
\int_{\mathcal{Y}_{S}}\left(\lambda \operatorname{div}_{\mathbf{y}} \chi^{0} \operatorname{Id}+2 \mu e_{\mathbf{y}}\left(\chi^{0}\right)\right): e_{\mathbf{y}}\left(\mathbf{v}^{1}\right)=\int_{\Gamma} \mathbf{v}^{1} \cdot \mathbf{n}^{S} .
\end{array}\right.
$$

These problems are elliptic thanks to Korn's inequality on $\mathcal{Y}_{S}$, so it is standard to show that they have a unique solution.

Macroscopic homogenized problem We now use the decomposition (3.39) to write the homogenized problem uniquely in terms of $\mathbf{u}$ and $\pi$. Using test functions $\mathbf{v} \in \mathbf{V}$ and $\phi \in L^{2}(\Omega)$ with $\mathbf{v}^{1}=\mathbf{0}$ in (3.34), we find that $\mathbf{u}_{\varepsilon}$ and $\pi$ satisfy the following identity:

$$
\begin{aligned}
\frac{\mathrm{d}}{\mathrm{d} t}\left\langle\theta \rho \partial_{t} \mathbf{u}, \mathbf{v}\right\rangle+ & \int_{\Omega} e_{\mathbf{x}}(\mathbf{u})_{k l}\left(\int_{\mathcal{Y}_{S}} \lambda \operatorname{div}_{\mathbf{y}}\left(\mathbf{p}^{k l}+\chi^{k l}\right) \operatorname{Id}+2 \mu e_{\mathbf{y}}\left(\mathbf{p}^{k l}+\chi^{k l}\right)\right): e_{\mathbf{x}}(\mathbf{v}) \\
- & \frac{\mathrm{d}}{\mathrm{d} t} \int_{\Omega} \pi\left((1-\theta) \operatorname{Id}+\int_{\mathcal{Y}_{S}} \lambda \operatorname{div}_{\mathbf{y}} \chi^{0} \operatorname{Id}+2 \mu e_{\mathbf{y}}\left(\chi^{0}\right)\right): e_{\mathbf{x}}(\mathbf{v}) \\
= & \int_{\Omega} \theta \mathbf{f} \cdot \mathbf{v}+\int_{\Gamma_{N}} p_{N} \mathbf{v} \cdot \mathbf{n} \\
& \quad-\int_{\Omega} p_{e}\left((1-\theta) \operatorname{Id}+\int_{\mathcal{Y}_{S}}\left(\lambda \operatorname{div}_{\mathbf{y}} \chi^{0} \operatorname{Id}+2 \mu e_{\mathbf{y}}\left(\chi^{0}\right)\right)\right): e_{\mathbf{x}}(\mathbf{v}),
\end{aligned}
$$

and by using the expression (3.39) of $\mathbf{u}^{1}$ in equation (3.37):

$$
\pi=-\mathcal{R}\left(\sum_{k, l=1}^{d} e_{\mathbf{x}}(\mathbf{u})_{k l}\left((1-\theta) \delta_{k l}-\int_{\Gamma} \chi^{k l} \cdot \mathbf{n}^{S}\right)+\left(\partial_{t} \pi-p_{e}\right) \int_{\Gamma} \chi^{0} \cdot \mathbf{n}^{S}\right) .
$$

These expressions motivate the introduction of the homogenized coefficients: the fourth-order elasticity tensor

$$
\mathcal{A}_{i j k l}^{h o m}=\int_{\mathcal{Y}_{S}}\left(\lambda \operatorname{div}_{\mathbf{y}}\left(\mathbf{p}^{k l}+\chi^{k l}\right) \operatorname{Id}+2 \mu e_{\mathbf{y}}\left(\mathbf{p}^{k l}+\chi^{k l}\right)\right)_{i j},
$$

the cell relaxation constant

$$
\tau_{h o m}=\int_{\Gamma} \chi^{0} \cdot \mathbf{n}^{S}
$$


and the fluid flux matrix

$$
B_{i j}^{h o m}=(1-\theta) \delta_{i j}-\int_{\Gamma} \chi^{i j} \cdot \mathbf{n}^{S} .
$$

The following properties of the homogenized coefficients are classical:

Proposition 3.6. The fourth-order tensor $\mathcal{A}^{\text {hom }}$ defined in (3.42) has the following properties:

a) Symmetry:

$$
\mathcal{A}_{i j k l}^{\text {hom }}=\mathcal{A}_{\text {klij }}^{\text {hom }}=\mathcal{A}_{\text {ijlk }}^{\text {hom }} .
$$

b) Ellipticity: there exists $\beta>0$ such that for any $d \times d$ symmetric matrix $\xi$,

$$
\left(\mathcal{A}^{h o m} \xi\right): \xi \geq \beta \xi: \xi \text {. }
$$

c) Positive definiteness:

$$
\mathcal{A}^{\text {hom }} \xi: \xi=0 \text { iff } \xi=0 .
$$

Moreover, the cell relaxation constant defined by (3.43) satisfies

$$
\tau_{\text {hom }}>0,
$$

and the matrix $B^{\text {hom }}$ defined by (3.44) is symmetric, and its entries satisfy:

$$
B_{i j}^{h o m}=(1-\theta) \delta_{i j}-\int_{\Gamma} \chi^{i j} \cdot \mathbf{n}^{S}=(1-\theta) \delta_{i j}+\int_{\mathcal{Y}_{S}}\left(\lambda \operatorname{div}_{\mathbf{y}} \chi^{0} \operatorname{Id}+2 \mu e_{\mathbf{y}}\left(\chi^{0}\right)\right)_{i j} .
$$

Remark 3.2. The coefficients $\mathcal{A}^{\text {hom }}$ and $\mathcal{B}^{\text {hom }}$ appear also in the homogenization of porous elastic solids filled with slightly viscous fluids, see [35]. In our case, we do not require a particular scaling of the fluid viscosity but the abstract tree convergence (2.12) to preserve a relative motion through the homogenization process, described here by the new macroscopic pressure $\partial_{t} \pi$.

Proof. The properties of $\mathcal{A}^{\text {hom }}$ and $\mathcal{B}^{\text {hom }}$ are classical and we do not reproduce the proof here, see e.g. Lemma 5.1 in [35] or [32].

To show that $\tau_{\text {hom }}$ is positive, we compute, using $\chi^{0}$ as a test function in the cell problem (3.41),

$$
\begin{aligned}
\tau_{\text {hom }} & =\int_{\Gamma} \chi^{0} \cdot \mathbf{n}^{S} \\
& =\int_{\mathcal{Y}_{S}}\left(\lambda \operatorname{div}_{\mathbf{y}} \chi^{0} \operatorname{Id}+2 \mu e_{\mathbf{y}}\left(\chi^{0}\right)\right): e_{\mathbf{y}}\left(\chi^{0}\right) \\
& =\int_{\mathcal{Y}_{S}} \lambda\left(\operatorname{div}_{\mathbf{y}}\left(\chi^{0}\right)^{2}+2 \mu e_{\mathbf{y}}\left(\chi^{0}\right): e_{\mathbf{y}}\left(\chi^{0}\right)\right. \\
& \geq 2 \mu\left\|e_{\mathbf{y}}\left(\chi^{0}\right)\right\|_{0, \mathcal{Y}_{S}}^{2} .
\end{aligned}
$$

Since $\boldsymbol{\chi}^{0} \neq \mathbf{0}$ and Korn's inequality holds on $\mathbf{H}_{\#}^{1}\left(\mathcal{Y}_{S}\right)$, we have $\tau_{\text {hom }}>0$. 
Using these homogenized parameters, we are able to write and describe the macroscopic limit problem verified by $(\mathbf{u}, \pi)$ when the tree operator converges:

Theorem 3.2. Suppose (3.1) and (2.12) hold. Then the pair $(\mathbf{u}, \pi)$ is the unique weak solution in the space $L^{\infty}(0, T ; \mathbf{V}) \cap W^{1, \infty}(0, T ; \mathbf{X}) \times H^{1}\left(0, T ; L^{2}(\Omega)\right)$ of the coupled homogenized problem:

$$
\left\{\begin{array}{lr}
\theta \rho \partial_{t t} \mathbf{u}-\operatorname{div} \sigma^{\text {hom }}(\mathbf{u})=\theta \mathbf{f}, & \text { in }(0, T) \times \Omega, \\
\pi+\tau_{\text {hom }} \partial_{t}(\mathcal{R} \pi)=\mathcal{R}\left(\tau_{\text {hom }} p_{e}-\mathcal{B}^{\text {hom }}: e(\mathbf{u})\right), & \text { in }(0, T) \times \Omega, \\
\sigma^{\text {hom }}(\mathbf{u}) \mathbf{n}=-\left(p_{N} \operatorname{Id}-p_{e} \mathcal{B}^{\text {hom }}\right) \mathbf{n}, & \text { on }(0, T) \times \Gamma_{N}, \\
\mathbf{u}=\mathbf{0}, & \text { on }(0, T) \times \Gamma_{D},
\end{array}\right.
$$

where $\sigma^{\text {hom }}(\mathbf{u})=\mathcal{A}^{\text {hom }} e(\mathbf{u})-\partial_{t} \pi \mathcal{B}^{\text {hom }}$, with the initial conditions:

$$
\mathbf{u}(0)=\mathbf{u}_{0}, \quad \partial_{t} \mathbf{u}(0)=\mathbf{u}_{1}, \quad \pi(0)=-(1-\theta) \mathcal{R}\left(\operatorname{div}_{\mathbf{x}} \mathbf{u}_{0}\right), \quad \text { in } \Omega .
$$

Remark 3.3. We recognize in the system (3.45) the usual linearized elasticity equation perturbed by a viscous pressure term. Observe that the effective elastic tensor $\mathcal{A}^{\text {hom }}$ is the same as for the elliptic (static) case without a tree, see [1,3]. The input of the tree appears through the evolution of the pressure field $\partial_{t} \pi$. Eq. (3.45b) describes a nonlocal relaxation effect on the fluid pressure and also induces dissipation of energy in the material. Hence the homogenized material behaves like a viscoelastic material with fading memory depending on the history of displacement, see e.g. $[12,20]$ but an unusual one since the dissipation is non-local.

Remark 3.4. We can make a few observations on the physical behavior of the homogenized material. There is no added mass effect from the fluid onto the structure, as it can be seen in the inertial term which is the same as in the original formulation (3.2). In addition, the instantaneous elastic response of the material differs from the static case presented in [3], because the compressibility of the air in the alveoli is not taken into account here: if the air does not have time to escape through the tree, each air cavity acts as if it is incompressible.

Proof. We know by construction that $(\mathbf{u}, \pi)$ satisfy the homogenized problem (3.45). Hence, we do not have to prove existence of a solution of the homogenized problem. Let us check that this solution is unique. Let $\left(\mathbf{u}_{1}, \pi_{1}\right)$ and $\left(\mathbf{u}_{2}, \pi_{2}\right)$ in $L^{\infty}(0, T ; \mathbf{V}) \cap W^{1, \infty}(0, T ; \mathbf{X}) \times H^{1}\left(0, T ; L^{2}(\Omega)\right)$ be two weak solutions of problem (3.45) with the same initial conditions. Then the difference $(\mathbf{w}, \psi)=$ $\left(\mathbf{u}_{1}-\mathbf{u}_{2}, \pi_{1}-\pi_{2}\right)$ satisfies, for all $\mathbf{v} \in \mathcal{D}([0, T) ; \mathbf{V})$ and $\phi \in \mathcal{D}\left([0, T) ; L^{2}(\Omega)\right)$ :

$$
\begin{array}{r}
\int_{0}^{T} \int_{\Omega}-\theta \rho \partial_{t} \mathbf{w} \cdot \partial_{t} \mathbf{v}+\left(\mathcal{A}^{\text {hom }} e(\mathbf{w})-\partial_{t} \psi \mathcal{B}^{\text {hom }}\right): e(\mathbf{v})=\mathbf{0}, \\
\int_{0}^{T} \int_{\Omega} \psi \phi+\mathcal{R}\left(\tau_{\text {hom }} \partial_{t} \psi+\mathcal{B}_{\text {hom }}: e(\mathbf{w})\right) \phi=0 .
\end{array}
$$


By a density argument, this variational formulation is also valid for $\mathbf{v} \in H^{1}(0, T ; \mathbf{V})$ with $\mathbf{v}(T)=0$ and $\phi \in L^{2}((0, T) \times \Omega)$. Let $s \in(0, T)$. We introduce the following test functions in the variational formulation (3.46):

$$
\begin{aligned}
& \mathbf{v}(t)=\left\{\begin{array}{ll}
\int_{t}^{s} \mathbf{w} & \text { if } t \leq s, \\
\mathbf{0} & \text { else, }
\end{array} \quad \text { in } H^{1}(0, T ; \mathbf{V}),\right. \\
& \phi=\tau_{\text {hom }} \partial_{t} \psi+\mathcal{B}_{\text {hom }}: e(\mathbf{w}) \quad \text { in } L^{2}((0, T) \times \Omega) .
\end{aligned}
$$

Since $\psi(0)=0, \mathbf{w}(0)=\mathbf{0}$ and $\partial_{t} \mathbf{v}(t)=\mathbf{w}(t)$ for $0 \leq t \leq s$, this yields:

$$
\begin{aligned}
& \int_{0}^{s} \int_{\Omega} \theta \rho \partial_{t} \mathbf{w} \cdot \mathbf{w}-\mathcal{A}^{h o m} e\left(\partial_{t} \mathbf{v}\right): e(\mathbf{v})-\psi \mathcal{B}^{h o m}: e(\mathbf{w})=\mathbf{0} \\
& \int_{0}^{s} \int_{\Omega} \tau_{h o m} \pi \partial_{t} \psi+\psi \mathcal{B}_{h o m}: e(\mathbf{w})+\mathcal{R}(\phi) \phi=0 .
\end{aligned}
$$

Adding the two equations, we obtain:

$$
\frac{1}{2} \int_{0}^{s} \frac{\mathrm{d}}{\mathrm{d} t}\left(\theta \rho\|\mathbf{w}\|_{0, \Omega}^{2}+\tau_{h o m}\|\psi\|_{0, \Omega}^{2}-\int_{\Omega} \mathcal{A}^{h o m} e(\mathbf{v}): e(\mathbf{v})\right)+\int_{0}^{T} \int_{\Omega} \mathcal{R}(\phi) \phi=0 .
$$

Using the fact that $\psi(0)=\mathbf{0}, \mathbf{w}(0)=0, \mathbf{v}(T)=\mathbf{0}$ and the positivity of the operator $\mathcal{R}$, we obtain:

$$
\theta \rho\|\mathbf{w}(s)\|_{0, \Omega}^{2}+\tau_{h o m}\|\psi(s)\|_{0, \Omega}^{2}+\int_{\Omega} \mathcal{A}^{h o m} e(\mathbf{v}(0)): e(\mathbf{v}(0)) \leq 0 .
$$

Since $s$ is arbitrary, $\mathbf{w}$ and $\psi$ are identically equal to zero.

\section{Study in the incompressible case}

We now turn to the study of the case where the elastic media is assumed to be incompressible. This hypothesis is nearly satisfied in the human lungs' case. To the displacement, we add a new unknown, the pressure $\eta_{\varepsilon}$, which is the Lagrange multiplier associated with the incompressibility constraint (not to be confused with the fluid pressure, which is related to $\pi_{\varepsilon}$ ). The model now reads as the system $(1.15)$ and we recall that the stress tensor is now defined by:

$$
\sigma\left(\mathbf{u}_{\varepsilon}, \eta_{\varepsilon}\right)=-\eta_{\varepsilon} \operatorname{Id}+2 \mu e\left(\mathbf{u}_{\varepsilon}\right) .
$$

We proceed as in the previous Sec. 3 and use the same notations: first, we write the mixed variational formulation and prove that problem (1.15) is well-posed and the solutions satisfy a priori bounds independently of $\varepsilon$; then, we use the two-scale convergence method in order to pass to the limit as $\varepsilon$ goes to zero; and finally we exhibit the homogenized model.

\subsection{Mixed variational formulation}

Let $M_{\varepsilon}=L^{2}\left(\Omega_{\varepsilon}\right), M=\left\{\phi \in L^{2}(\Omega) \mid \int_{\Omega} \phi=0\right\}, \mathbf{V}_{\varepsilon}^{i n c}=\left\{\mathbf{v}_{\varepsilon} \in \mathbf{V}_{\varepsilon} \mid \operatorname{div}\left(\mathbf{v}_{\varepsilon}\right)=\right.$ 0 a.e. $\left.\mathbf{x} \in \Omega_{\varepsilon}\right\}$ and $\mathbf{V}^{i n c}=\{\mathbf{v} \in \mathbf{V} \mid \operatorname{div}(\mathbf{v})=0$ a.e. $\mathbf{x} \in \Omega\}$. 
We make the following assumptions, in similar way as we did for the compressible case:

$$
\mathbf{f} \in L^{2}((0, T) ; \mathbf{X}), \quad p_{N}, p_{e} \in H^{1}(0, T), \quad \mathbf{u}_{0} \in \mathbf{V}^{i n c} \text { and } \mathbf{u}_{1} \in L^{2}(\Omega) .
$$

The mixed variational formulation associated to (2.4) is as follows. Find a pair $\mathbf{u}_{\varepsilon} \in L^{\infty}\left(0, T ; \mathbf{V}_{\varepsilon}\right)$ and $\eta_{\varepsilon} \in H^{-1}\left(0, T ; M_{\varepsilon}\right)$ such that:

$$
\left\{\begin{array}{cc}
\text { For all } \mathbf{v}_{\varepsilon} \in \mathbf{V}_{\varepsilon}, \quad \phi_{\varepsilon} \in M_{\varepsilon}, & \\
\frac{\mathrm{d}}{\mathrm{d} t}\left(\rho \partial_{t} \mathbf{u}_{\varepsilon}, \mathbf{v}_{\varepsilon}\right)_{0, \Omega_{\varepsilon}}+\frac{\mathrm{d}}{\mathrm{d} t} r_{\varepsilon}\left(\mathbf{u}_{\varepsilon}, \mathbf{v}_{\varepsilon}\right) & \text { in } \mathcal{D}^{\prime}(0, T), \\
+a_{\varepsilon}^{i n c}\left(\mathbf{u}_{\varepsilon}, \mathbf{v}_{\varepsilon}\right)+b_{\varepsilon}^{i n c}\left(\eta_{\varepsilon}, \mathbf{v}_{\varepsilon}\right)=\ell_{\varepsilon}\left(\mathbf{v}_{\varepsilon}\right), & \\
b_{\varepsilon}^{i n c}\left(\phi_{\varepsilon}, \mathbf{u}_{\varepsilon}\right)=0, & \\
\mathbf{u}_{\varepsilon}(0)=\mathbf{u}_{0} \quad \text { and } \quad \partial_{t} \mathbf{u}_{\varepsilon}(0)=\mathbf{u}_{1}, &
\end{array}\right.
$$

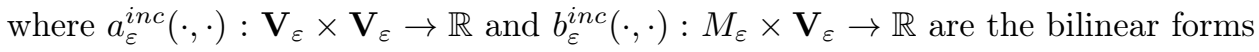
defined by:

$$
\begin{aligned}
a_{\varepsilon}^{i n c}\left(\mathbf{u}_{\varepsilon}, \mathbf{v}_{\varepsilon}\right) & =\int_{\Omega_{\varepsilon}} 2 \mu e\left(\mathbf{u}_{\varepsilon}\right): e\left(\mathbf{v}_{\varepsilon}\right), \\
b_{\varepsilon}^{i n c}\left(\phi, \mathbf{v}_{\varepsilon}\right) & =-\int_{\Omega_{\varepsilon}} \phi \operatorname{div}\left(\mathbf{v}_{\varepsilon}\right),
\end{aligned}
$$

and the forms $r_{\varepsilon}(\cdot, \cdot), \ell_{\epsilon}(\cdot)$ are as in the Section 3, defined in (3.4) and (3.5) respectively.

\subsection{Pressure extension and a priori estimates}

The variational formulation (4.2) of the system (1.15) is similar to the variational formulation (3.2) studied in Section 3 but for the introduction of the pressure term. We introduce an extension operator for the pressure defined on $\Omega_{\varepsilon}$ as follows. Given $\eta_{\varepsilon} \in M_{\varepsilon}$, we extend it by (see $\left.[3,10]\right)$ :

$$
\widetilde{\eta}_{\varepsilon}(\mathbf{x})= \begin{cases}\eta_{\varepsilon}(\mathbf{x}) & \text { if } \mathbf{x} \in \Omega_{\varepsilon}, \\ -\frac{1}{\left|\Omega \backslash \Omega_{\varepsilon}\right|} \int_{\Omega_{\varepsilon}} \eta_{\varepsilon}(\mathbf{x}) \mathrm{d} \mathbf{x} & \text { if } \mathbf{x} \in \Omega \backslash \Omega_{\varepsilon} .\end{cases}
$$

This extension is such that $\widetilde{\eta}_{\varepsilon} \in M$, and:

$$
\left\|\widetilde{\eta_{\varepsilon}}\right\|_{M} \leq C\left\|\eta_{\varepsilon}\right\|_{M_{\varepsilon}}
$$

with $C$ independent of $\varepsilon$. We have the following existence and uniqueness result:

Proposition 4.1. Under hypothesis (4.1) and (2.12), the problem (1.15) has a unique weak solution $\left(\mathbf{u}_{\varepsilon}, \eta_{\varepsilon}\right)$, which satisfies:

$$
\begin{gathered}
\mathbf{u}_{\varepsilon} \in L^{\infty}\left(0, T ; \mathbf{V}_{\varepsilon}\right) \quad \text { and } \quad \partial_{t} \mathbf{u}_{\varepsilon} \in L^{\infty}\left(0, T ; \mathbf{X}_{\varepsilon}\right), \\
\eta_{\varepsilon} \in H^{-1}\left(0, T ; M_{\varepsilon}\right) .
\end{gathered}
$$


In addition, the sequence of extended solutions $\left(\widehat{\mathbf{u}_{\varepsilon}}, \widetilde{\eta_{\varepsilon}}\right)$ satisfies the a priori bounds:

$$
\begin{aligned}
\left|\widehat{\mathbf{u}_{\varepsilon}}\right|_{1, \Omega}^{2}+\left\|\widehat{\partial_{t} \mathbf{u}_{\varepsilon}}\right\|_{0, \Omega}^{2}+\mathcal{V}_{\varepsilon}(t) & \leq C, \text { a.e. } t \in[0, T], \\
\left\|\widetilde{\eta}_{\varepsilon}\right\|_{H^{-1}(0, T ; M)} & \leq C,
\end{aligned}
$$

for some $C>0$ independent of $\varepsilon$, where $\mathcal{V}_{\varepsilon}(t)$ is defined as in Section 3 by (3.8).

Proof. As a first step, we study the auxiliary variational formulation obtained by using only divergence-free test functions in (4.2a): find $\mathbf{u}_{\varepsilon} \in L^{\infty}\left(0, T ; \mathbf{V}_{\varepsilon}^{\text {inc }}\right)$ such that

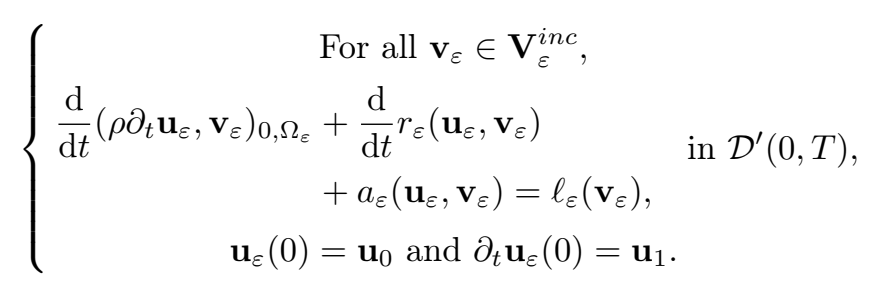

By using the classical Galerkin method, as in the compressible case (see [38]), we can prove that there exists a unique solution to the auxiliary variational problem (4.6) such that (4.4a) and (4.5a) hold.

Next, we prove the existence of a pressure $\eta_{\varepsilon}$ in $H^{-1}\left(0, T ; M_{\varepsilon}\right)$ such that the full formulation (4.2) is satisfied. Integrating (4.6) in time we see that for a.e. $t \in(0, T)$

$$
\left\{\begin{array}{rl}
\left(\rho \partial_{t} \mathbf{u}_{\varepsilon}, \mathbf{v}_{\varepsilon}\right)_{0, \Omega_{\varepsilon}} & +r_{\varepsilon}\left(\mathbf{u}_{\varepsilon}, \mathbf{v}_{\varepsilon}\right)-\left(\rho \mathbf{u}_{1}, \mathbf{v}_{\varepsilon}\right)_{0, \Omega_{\varepsilon}}-r_{\varepsilon}\left(\mathbf{u}_{0}, \mathbf{v}_{\varepsilon}\right) \\
& +a_{\varepsilon}\left(\int_{0}^{t} \mathbf{u}_{\varepsilon}, \mathbf{v}_{\varepsilon}\right)-\int_{0}^{t} \ell_{\varepsilon}\left(\mathbf{v}_{\varepsilon}\right)=0
\end{array} \quad \forall \mathbf{v}_{\varepsilon} \in \mathbf{V}_{\varepsilon}^{i n c} .\right.
$$

Using classical arguments (see e.g. [17]) we know then that a.e. $t \in(0, T)$, there exists a unique Lagrange multiplier $P_{\varepsilon}(t) \in L^{2}\left(\Omega_{\varepsilon}\right)=M_{\varepsilon}$ such that

$$
\left\{\begin{array}{rl}
\left(\rho \partial_{t} \mathbf{u}_{\varepsilon}(t), \mathbf{v}_{\varepsilon}\right)_{0, \Omega_{\varepsilon}} & +r_{\varepsilon}\left(\mathbf{u}_{\varepsilon}(t), \mathbf{v}_{\varepsilon}\right)-\left(\rho \mathbf{u}_{1}, \mathbf{v}_{\varepsilon}\right)_{0, \Omega_{\varepsilon}}-r_{\varepsilon}\left(\mathbf{u}_{0}, \mathbf{v}_{\varepsilon}\right) \\
& +a_{\varepsilon}\left(\int_{0}^{t} \mathbf{u}_{\varepsilon}, \mathbf{v}_{\varepsilon}\right)-\int_{0}^{t} \ell_{\varepsilon}\left(\mathbf{v}_{\varepsilon}\right)=\int_{\Omega_{\varepsilon}} P_{\varepsilon}(t) \operatorname{div}\left(\mathbf{v}_{\varepsilon}\right)
\end{array} \forall \mathbf{v}_{\varepsilon} \in \mathbf{V}_{\varepsilon} .\right.
$$

Moreover, there exists a constant $C$, which can be shown to be independent of $\Omega_{\varepsilon}$ and dependent only on $\Omega$ (see e.g. [3]) such that a.e. $t \in(0, T)$

$$
\begin{gathered}
\left\|P_{\varepsilon}(t)\right\|_{M_{\varepsilon}} \leq C \\
\left(\left\|\mathbf{u}_{\varepsilon}\right\|_{L^{\infty}\left(0, T ; \mathbf{V}_{\varepsilon}\right)}+\left\|\partial_{t} \mathbf{u}_{\varepsilon}(t)\right\|_{0, \Omega}+\left\|\mathbf{u}_{0}\right\|_{\mathbf{V}^{i n c}}+\left\|\mathbf{u}_{1}\right\|_{L^{2}(\Omega)}\right. \\
\left.+\|\mathbf{f}\|_{L^{2}((0, T) ; \mathbf{X})}+\left\|p_{N}\right\|_{H^{1}(0, T)}+\left\|p_{e}\right\|_{H^{1}(0, T)}\right) .
\end{gathered}
$$

It is then a consequence of $(4.5 \mathrm{a})$ that $P_{\varepsilon}$ belongs to $L^{2}\left(0, T ; M_{\varepsilon}\right)$ and satisfies a uniform bound with respect to $\varepsilon$. Let us define $\eta_{\varepsilon}=\partial_{t} P_{\varepsilon} \in H^{-1}\left(0, T ; \mathbf{V}_{\varepsilon}\right)$. Then it is clear that the pair $\left(\mathbf{u}_{\varepsilon}, \eta_{\varepsilon}\right)$ is the unique solution of the problem (4.2) and furthermore $\eta_{\varepsilon}$ satisfies the uniform bound (4.5b). 


\subsection{Two-scale convergence}

As in the compressible case, we apply the two-scale convergence method to find the homogenized problem. To deal with the pressure term we define the two-scale convergence in $H^{-1}(0, T)$ as follows:

Definition 4.1. Let $\left(\phi_{\varepsilon}\right)_{\varepsilon>0}$ be a sequence in $H^{-1}(0, T ; M)$. We say that $\phi_{\varepsilon}$ twoscale converges to $\phi$ in $H^{-1}\left(0, T ; L_{0}^{2}(\Omega \times \mathcal{Y})\right)$ when:

$$
\begin{gathered}
\forall \psi(t, \mathbf{x}, \mathbf{y}) \in H_{0}^{1}\left((0, T) ; L^{2}\left(\Omega ; C_{\#}(\mathcal{Y})\right)\right) \\
\lim _{\varepsilon \rightarrow 0} \int_{0}^{T} \int_{\Omega} \phi_{\varepsilon}(t, \mathbf{x}) \psi\left(t, \mathbf{x}, \frac{\mathbf{x}}{\varepsilon}\right) \mathrm{d} \mathbf{x}=\int_{0}^{T} \int_{\Omega} \int_{Y} \phi(t, \mathbf{x}, \mathbf{y}) \psi(t, \mathbf{x}, \mathbf{y}) \mathrm{d} \mathbf{y} \mathrm{d} \mathbf{x} .
\end{gathered}
$$

Since the time variable is only a parameter in the two-scale convergence defined by $(4.7)$ and $H^{-1}(0, T)$ is separable, the weak two-scale convergence defined by (4.7) has the same compactness property as in the square-integrable in time case (Lemma 3.2).

\subsubsection{Convergence of the sequence of solutions}

As in the compressible case, we define $q_{\varepsilon}$ and $\pi_{\varepsilon}$ as:

$$
\begin{array}{r}
q_{\varepsilon}=-\Pi_{\varepsilon}\left(\chi_{F, \varepsilon} \operatorname{div} \widehat{\mathbf{u}_{\varepsilon}}\right) \in H^{1}\left(0, T ; L^{2}(\Omega)\right) \\
\pi_{\varepsilon}=\mathcal{R}_{\varepsilon} q_{\varepsilon} \in H^{1}\left(0, T ; L^{2}(\Omega)\right) .
\end{array}
$$

We obtain also the convergence results:

Proposition 4.2. Suppose (4.1) and (2.12) hold. Then, there exists $\mathbf{u} \in$ $L^{2}(0, T ; \mathbf{V}), \mathbf{u}^{1} \in L^{2}\left((0, T) \times \Omega ; \mathbf{H}_{\#}^{1}(\mathcal{Y}) / \mathbb{R}^{d}\right)$ and $\eta \in H^{-1}(0, T ; M)$ such that $\partial_{t} \mathbf{u} \in L^{2}(0, T ; \mathbf{X})$ and up to a subsequence, still denoted by $\varepsilon$,

$$
\left\{\begin{array}{rlr}
\widehat{\mathbf{u}_{\varepsilon}} & \rightarrow \mathbf{u} \\
\nabla \widehat{\mathbf{u}_{\varepsilon}} & \rightarrow \nabla_{x} \mathbf{u}+\nabla_{y} \mathbf{u}^{1} & \text { two-scale in } L^{2}((0, T) \times \Omega \times \mathcal{Y}), \\
\widehat{\partial_{t} \mathbf{u}_{\varepsilon}} & \rightarrow \partial_{t} \mathbf{u} & \\
\widetilde{\eta}_{\varepsilon} & \rightarrow \eta & \text { two-scale in } H^{-1}\left(0, T ; L^{2}(\Omega \times \mathcal{Y})\right) .
\end{array}\right.
$$

There exists $q \in L^{2}\left(0, T ; L^{2}(\Omega)\right), \pi \in H^{1}\left(0, T ; L^{2}(\Omega)\right)$ such that:

$$
\begin{array}{rlr}
q_{\varepsilon} & \rightarrow q, & \text { weakly in } L^{2}((0, T) \times \Omega), \\
\pi_{\varepsilon}=\mathcal{R}_{\varepsilon} q_{\varepsilon} & \rightarrow \pi=\mathcal{R} q & \text { strongly in } L^{2}((0, T) \times \Omega), \\
\partial_{t} \pi_{\varepsilon}=\mathcal{R}_{\varepsilon} \partial_{t} q_{\varepsilon} & \rightarrow \partial_{t} \pi=\partial_{t}(\mathcal{R} q) & \text { weakly in } L^{2}((0, T) \times \Omega) .
\end{array}
$$




\subsubsection{The two-scale limit problem}

Next, we deduce from Prop. 4.2 the asymptotic two-scale formulation satisfied by $\mathbf{u}, \mathbf{u}^{1}$ and $\eta$ as in Sec. 3:

$$
\left\{\begin{array}{c}
\text { For all } \mathbf{v} \in \mathbf{V}, \mathbf{v}^{1} \in \mathbf{H}, \phi \in L^{2}\left(\Omega \times \mathcal{Y}_{S}\right), \\
\frac{\mathrm{d}}{\mathrm{d} t}\left(\theta \rho \partial_{t} \mathbf{u}, \mathbf{v}\right)_{0, \Omega}+\frac{\mathrm{d}}{\mathrm{d} t} r_{\#}\left(\left(\mathbf{u}, \mathbf{u}^{1}\right),\left(\mathbf{v}, \mathbf{v}^{1}\right)\right) \\
+a_{\#}^{\text {inc }}\left(\left(\mathbf{u}, \mathbf{u}^{1}\right),\left(\mathbf{v}, \mathbf{v}^{1}\right)\right)+b_{\#}^{i n c}\left(\eta,\left(\mathbf{v}, \mathbf{v}^{1}\right)\right)=\ell_{\#}\left(\mathbf{v}, \mathbf{v}^{1}\right), \\
b_{\#}^{\text {inc }}\left(\phi,\left(\mathbf{u}, \mathbf{u}^{1}\right)\right)=0,
\end{array} \text { in } \mathcal{D}^{\prime}(0, T),\right.
$$

with the initial conditions

$$
\left\{\begin{array}{l}
\mathbf{u}(0)=\mathbf{u}_{0}, \partial_{t} \mathbf{u}(0)=\mathbf{u}_{1}, \\
\pi(0)=\left.\mathcal{R}\left((1-\theta) \operatorname{div}_{\mathbf{x}} \mathbf{u}-\int_{\Gamma} \mathbf{u}^{1} \cdot \mathbf{n}^{S}\right)\right|_{t=0}=0,
\end{array}\right.
$$

where we have used the bilinear form $a_{\#}^{i n c}$ on $\mathbf{V} \times \mathbf{H}$ and the mixed bilinear form $b_{\#}^{\text {inc }}$ on $L^{2}\left(\Omega \times \mathcal{Y}_{S}\right) \times(\mathbf{V} \times \mathbf{H})$ defined by:

$$
\begin{aligned}
a_{\#}^{i n c}\left(\left(\mathbf{u}, \mathbf{u}^{1}\right),\left(\mathbf{v}, \mathbf{v}^{1}\right)\right) & =\int_{\Omega} \int_{\mathcal{Y}_{S}} 2 \mu\left(e_{\mathbf{x}}(\mathbf{u})+e_{\mathbf{y}}\left(\mathbf{u}^{1}\right)\right):\left(e_{\mathbf{x}}(\mathbf{v})+e_{\mathbf{y}}\left(\mathbf{v}^{1}\right)\right), \\
b_{\#}^{\text {inc }}\left(\eta,\left(\mathbf{v}, \mathbf{v}^{1}\right)\right) & =-\int_{\Omega} \int_{\mathcal{Y}_{S}} \eta\left(\operatorname{div}_{\mathbf{x}} \mathbf{v}+\operatorname{div}_{\mathbf{y}} \mathbf{v}^{1}\right) .
\end{aligned}
$$

We recall that $r_{\#}$ and $\ell_{\#}$ are defined respectively by (3.32) and (3.33).

Performing the same computations as in the proof of Prop. 3.5, we also obtain the two key results:

Proposition 4.3. The variational problem (4.14) has a unique solution in the space of triplets $\left(\mathbf{u}, \mathbf{u}^{1}, \eta\right)$ such that

$$
\left\{\begin{array}{l}
\left.\mathbf{u} \in L^{2}(0, T ; \mathbf{V}) \cap H^{1}(0, T) ; \mathbf{X}\right) \\
\mathbf{u}^{1} \in L^{2}(0, T ; \mathbf{H}) \\
\pi=\mathcal{R}\left((1-\theta) \operatorname{div}_{\mathbf{x}} \mathbf{u}-\int_{\Gamma} \mathbf{u}^{1} \cdot \mathbf{n}^{S}\right) \in H^{1}\left(0, T ; L^{2}(\Omega)\right) \\
\eta \in H^{-1}\left(0, T ; L^{2}\left(\Omega \times \mathcal{Y}_{S}\right)\right)
\end{array}\right.
$$

Theorem 4.1. Suppose (2.12) and (4.1) hold. Let $\left(\mathbf{u}_{\varepsilon}, \eta_{\varepsilon}\right)_{\varepsilon>0}$ be the sequence of solutions of the family of problems (1.15) when $\varepsilon$ varies.

Then the full sequences $\left(\widehat{\mathbf{u}_{\varepsilon}} \chi_{S, \varepsilon}\right)_{\varepsilon>0}, \quad\left(\widehat{\partial_{t} \mathbf{u}_{\varepsilon}} \chi_{S, \varepsilon}\right)_{\varepsilon>0}, \quad\left(\nabla\left(\widehat{\mathbf{u}_{\varepsilon}}\right) \chi_{S, \varepsilon}\right)_{\varepsilon>0} \quad$ and $\left(\widetilde{\eta}_{\varepsilon} \chi_{S, \varepsilon}\right)_{\varepsilon>0}$ two-scale converge respectively to $\mathbf{u} \chi_{S}, \partial_{t} \mathbf{u} \chi_{S},\left(\nabla_{x} \mathbf{u}+\nabla_{y} \mathbf{u}^{1}\right) \chi_{S}$ in $L^{2}((0, T) \times \Omega \times \mathcal{Y})$ and $\eta \chi_{S}$ in $H^{-1}\left(0, T ; L^{2}(\Omega \times \mathcal{Y})\right)$, where $\left(\mathbf{u},\left.\mathbf{u}^{1}\right|_{\Omega \times \mathcal{Y}_{S}},\left.\eta\right|_{\Omega \times \mathcal{Y}_{S}}\right)$ is the unique solution of the two-scale variational problem (4.14).

In the following discussion, for simplicity, we denote $\mathbf{u}^{1}$ and $\eta$ their respective restriction to $\Omega \times \mathcal{Y}_{S}$, which is also equal to $\mathbf{u}^{1}$ and $\eta$. 


\subsubsection{Cell problems and the homogenized law}

As in the compressible case, we use appropriate test functions $\mathbf{v}, \mathbf{v}^{1}$ and $\phi$ to identify two strong differential problems from the two-scale variational problem (4.14), one in the microscopic variable and one in the macroscopic variable. Note that the two-scale incompressibility condition (4.14b) implies that

$$
\operatorname{div}_{\mathbf{x}} \mathbf{u}+\operatorname{div}_{\mathbf{y}} \mathbf{u}^{1}=0 \text { in } \Omega \times \mathcal{Y}_{S} .
$$

Then, the non-local viscous term can be written without the microscopic unknown $\mathbf{u}^{1}$ thanks to the resulting relations:

$$
\int_{\Gamma} \mathbf{u}^{1} \cdot \mathbf{n}^{S}=\int_{\mathcal{Y}_{S}} \operatorname{div}_{\mathbf{y}} \mathbf{u}^{1}=-\int_{\mathcal{Y}_{S}} \operatorname{div}_{\mathbf{x}} \mathbf{u}=-\theta \operatorname{div}_{\mathbf{x}} \mathbf{u} .
$$

As a consequence, we have the following expression:

$$
r_{\#}\left(\left(\mathbf{u}, \mathbf{u}^{1}\right),\left(\mathbf{v}, \mathbf{v}^{1}\right)\right)=\int_{\Omega}\left(\mathcal{R} \operatorname{div}_{\mathbf{x}} \mathbf{u}\right)\left((1-\theta) \operatorname{div}_{\mathbf{x}} \mathbf{v}-\int_{\Gamma} \mathbf{v}^{1} \cdot \mathbf{n}^{S}\right) .
$$

This is different from the compressible case, where the equivalent term couples the values of $\mathbf{u}^{1}$ globally across $\Omega$. Indeed, we can write directly the unknowns $q$ and $\pi$ defined in the compressible case by Prop. 3.3: we have

$$
q=-\operatorname{div}_{\mathbf{x}} \mathbf{u} \quad \text { and } \quad \pi=-\mathcal{R}\left(\operatorname{div}_{\mathbf{x}} \mathbf{u}\right) .
$$

Cell problem First, we write the local cell problem by taking the test function $\mathbf{v}$ to be 0 in the two-scale variational formulation (4.14a). Then $\mathbf{u}^{1}$ and $\eta$ are the solution of a mixed variational problem on $\mathcal{Y}_{S}$ parameterized by the macroscopic displacement $\mathbf{u}$ :

$$
\left\{\begin{aligned}
\text { For all }\left(\mathbf{v}^{1}, \phi\right) \in \mathbf{H}_{\#}^{1}\left(\mathcal{Y}_{S}\right) & \times L^{2}\left(\mathcal{Y}_{S}\right) \\
\int_{\mathcal{Y}_{S}} 2 \mu e_{\mathbf{y}}\left(\mathbf{u}^{1}\right) & : e_{\mathbf{y}}\left(\mathbf{v}^{1}\right)-\int_{\mathcal{Y}_{S}} \eta \operatorname{div}_{\mathbf{y}} \mathbf{v}^{1} \\
= & -\int_{\mathcal{Y}_{S}} 2 \mu e_{\mathbf{x}}(\mathbf{u}): e_{\mathbf{y}}\left(\mathbf{v}^{1}\right) \\
& +\left(\frac{\partial}{\partial t} \mathcal{R}\left(\operatorname{div}_{\mathbf{x}} \mathbf{u}\right)-p_{e}\right) \int_{\mathcal{Y}_{S}} \operatorname{div}_{\mathbf{y}} \mathbf{v}^{1} \\
\int_{\mathcal{Y}_{S}} \phi \operatorname{div}_{\mathbf{y}} \mathbf{u}^{1}= & -\int_{\mathcal{Y}_{S}} \phi \operatorname{div}_{\mathbf{x}} \mathbf{u} .
\end{aligned}\right.
$$

This is a standard cell problem where the variable $\mathbf{x}$ appears only as a parameter. Now, we introduce the correctors as is standard. Let us denote by $\left(\chi_{i n c}^{k l}, \eta^{k l}\right)$ the solutions of the auxiliary local problems:

$$
\left\{\begin{array}{rlrl}
-\operatorname{div}_{\mathbf{y}}\left(-\eta^{k l} \operatorname{Id}+2 \mu e_{\mathbf{y}}\left(\chi_{i n c}^{k l}\right)\right) & =\mathbf{0}, & & \text { in } \mathcal{Y}_{S}, \\
\operatorname{div}_{\mathbf{y}} \boldsymbol{\chi}_{i n c}^{k l} & =\delta_{k l}, & & \text { in } \mathcal{Y}_{S}, \\
\left(-\eta^{k l} \operatorname{Id}+2 \mu e_{\mathbf{y}}\left(\chi_{i n c}^{k l}\right)\right) \mathbf{n}^{S} & =-2 \mu e_{\mathbf{y}}\left(\mathbf{p}^{k l}\right) \mathbf{n}^{S}, & & \text { on } \Gamma, \\
\boldsymbol{\chi}_{i n c}^{k l}, \eta^{k l} \mathcal{Y} & \text {-periodic, } &
\end{array}\right.
$$


where $1 \leq k, l \leq d$ and $\mathbf{p}^{k l}$ is the polynomial introduced in (3.38). Using the superposition principle in (4.17) we have that:

$$
\begin{gathered}
\mathbf{u}^{1}=e_{\mathbf{x}}(\mathbf{u})_{k l} \chi_{i n c}^{k l} \\
\eta=e_{\mathbf{x}}(\mathbf{u})_{k l} \eta^{k l}-\left(\frac{\partial}{\partial t} \mathcal{R}\left(\operatorname{div}_{\mathbf{x}} \mathbf{u}\right)-p_{e}\right)
\end{gathered}
$$

Remark 4.1. If we compare the incompressible cell problem (3.40) to the compressible case (3.36), it is clear that there is a major simplification in the incompressible case: the problems are no longer coupled across space and time, thanks to the incompressibility condition (4.14b). This is reflected in the decomposition of $\mathbf{u}^{1}$ and $\eta$ on the basis of the correctors as in (4.19): we do not need the introduction of the fluid pressure unknown $\pi$ as in the compressible case (3.39). This simplification is a consequence of the coupling in our model of an incompressible structure with an incompressible fluid in the bronchial tree: information is propagated instantly and, as a result, the time delay term disappears compared to the compressible case.

Homogenized problem Thanks to the decomposition (4.19) we can now identify the macroscopic problem. Using a test function $\mathbf{v} \in \mathbf{V}$ with $\mathbf{v}^{1}$ and $\phi$ equal to zero in (4.14a), we obtain the following variational problem for $\mathbf{u}$ :

$$
\left\{\begin{array}{l}
\text { For all } \mathbf{v} \in \mathbf{V} \\
\frac{\mathrm{d}}{\mathrm{d} t}\left(\theta \rho \partial_{t} \mathbf{u}, \mathbf{v}\right)_{0, \Omega}+\frac{\mathrm{d}}{\mathrm{d} t} \int_{\Omega}(1-\theta) \mathcal{R}\left(\operatorname{div}_{\mathbf{x}} \mathbf{u}\right) \operatorname{div}_{\mathbf{x}} \mathbf{v} \\
\quad+\int_{\Omega} e_{\mathbf{x}}(\mathbf{u})_{k l}\left(\int_{\mathcal{Y}_{S}}-\eta^{k l} \mathrm{Id}+2 \mu e_{\mathbf{y}}\left(\mathbf{p}^{k l}+\chi_{i n c}^{k l}\right)\right): e_{\mathbf{x}}(\mathbf{v}) \quad \text { in } \mathcal{D}^{\prime}(0, T), \\
=\int_{\Omega} \theta \mathbf{f} \cdot \mathbf{v}+\int_{\Gamma_{N}}\left(p_{N}-p_{e}\right) \mathbf{v} \cdot \mathbf{n}, \\
\mathbf{u}(0)=\mathbf{u}_{0}, \partial_{t} \mathbf{u}(0)=\mathbf{u}_{1} \quad \text { in } \Omega .
\end{array}\right.
$$

Let us introduce the homogenized elasticity tensor:

$$
\left(\mathcal{A}_{i n c}^{h o m}\right)_{i j k l}=\int_{\mathcal{Y}_{S}}-\eta^{k l} \delta_{i j}+2 \mu e_{\mathbf{y}}\left(\mathbf{p}^{k l}+\chi_{i n c}^{k l}\right)_{i j}
$$

As in the compressible case, the tensor $\mathcal{A}_{\text {inc }}^{\text {hom }}$ has the following properties:

Proposition 4.4. The fourth-order tensor $\mathcal{A}_{\text {inc }}^{\text {hom }}$ defined in (4.20) is symmetric, elliptic, positive definite.

The proof is classical and follows the same line as in $[3,35]$. Then, we can describe the limit problem verified by the macroscopic displacement $\mathbf{u}$ as follows:

Theorem 4.2. Suppose that (2.12) and (4.1) hold. Then $\mathbf{u}$ is the unique weak solution in the space $L^{\infty}(0, T ; \mathbf{V}) \cap W^{1, \infty}(0, T ; \mathbf{X})$ of the following homogenized 
problem:

$$
\left\{\begin{array}{lr}
\theta \rho \partial_{t t} \mathbf{u}-\operatorname{div} \sigma_{\text {inc }}^{\text {hom }}(\mathbf{u})=\theta \mathbf{f} & \text { in }(0, T) \times \Omega, \\
\sigma_{\text {inc }}^{\text {hom }}(\mathbf{u}) \mathbf{n}=\left(p_{e}-p_{N}\right) \mathbf{n}, & \text { on }(0, T) \times \Gamma_{N}, \\
\mathbf{u}=\mathbf{0}, & \text { on }(0, T) \times \Gamma_{D},
\end{array}\right.
$$

where

$$
\sigma_{i n c}^{\text {hom }}(\mathbf{u})=\mathcal{A}_{i n c}^{\text {hom }} e(\mathbf{u})+(1-\theta) \partial_{t} \mathcal{R}(\operatorname{div} \mathbf{u}) \operatorname{Id},
$$

with the initial conditions:

$$
\mathbf{u}(0)=\mathbf{u}_{0}, \quad \partial_{t} \mathbf{u}(0)=\mathbf{u}_{1}, \quad \text { in } \Omega .
$$

The proof is exactly the same as in the compressible case.

Remark 4.2. Let us compare the compressible (3.45) and incompressible (4.21) homogenized problems. As in the static case [3], we can obtain the incompressible homogenized coefficients by taking the limit $\lambda \rightarrow \infty$ in the compressible cell problems (3.40) and (3.41). In particular, we see that $\tau_{\text {hom }}$ takes the value 0 in the incompressible case, which is why the memory effect in time disappears. The matrix $\mathcal{B}^{\text {hom }}$ also converges to $(1-\theta) \mathrm{Id}$.

Remark 4.3. In the study of a one-dimensional model for the lungs, the authors of [18] also obtain a non-local viscoelastic constitutive law with no fading memory effects. The extension of the tree embedding and of the tree operator $\mathcal{R}$ to a multidimensional framework is described in [39], where (4.21d) is proposed, without proof, as the constitutive law for an elastic material irrigated by a dyadic tree. Here we obtain rigorously a generalization of [18] in the three-dimensional context.

\section{A numerical strategy}

We present in this section the numerical approach we adopted to simulate a material which obeys the homogenized parenchyma models (3.45) or (4.21) obtained in the Sections 3 and 4 . The main difficulty is the numerical treatment of the non-local operator in space which appears in the homogenized problems, which is represented by a full matrix in the finite elements basis. In order to obtain an efficient numerical scheme, we propose to use an iterative method and to compute efficiently the matrixvector products associated to the non-local operator by algorithms which make use of the tree structure to obtain a linear complexity.

\subsection{Discretization of the homogenized problem}

We present first the discretization scheme of the homogenized system of equations. For simplicity, the volumic force $\mathbf{f}$ and the pressure at the root of the tree $p_{e}$ will be zero in this section, and the system is driven by the pressure applied on the 
external boundary $p_{N}$. Keeping the notations of the homogenized problem in the compressible case, we can write the problem as a first-order mixed coupled problem.

Recall that $\mathbf{u}$ is the displacement of the homogenized material, the unknown $\pi$ is a primitive in time of a pressure as the homogenized limit of the air pressure in the alveoli, and let us also introduce as an additional unknown the velocity, denoted $\mathbf{v}$, of the homogenized material. Then the triplet $(\mathbf{u}, \mathbf{v}, \pi)$ satisfies the following formulation:

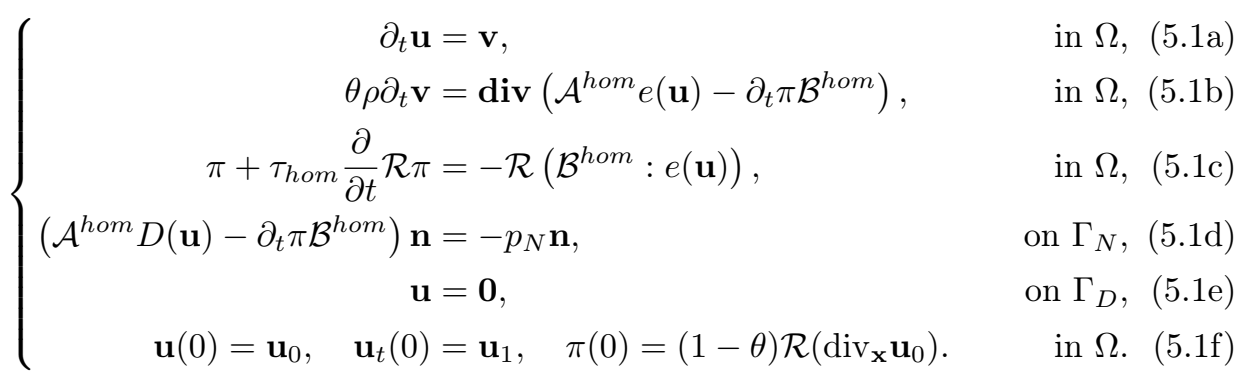

Remark 5.1. This formulation holds also in the incompressible case, where the parameters $\mathcal{A}^{\text {hom }}, \mathcal{B}^{\text {hom }}$ and $\tau_{\text {hom }}$ are identified as:

$$
\mathcal{A}^{\text {hom }}=\mathcal{A}_{\text {inc }}^{\text {hom }}, \quad \mathcal{B}^{\text {hom }}=(1-\theta) \mathrm{Id}, \quad \tau_{\text {hom }}=0 .
$$

Time discretization We introduce $\Delta t>0$ a timestep, $t_{n}=n \Delta t$ and, for any vector $\mathbf{w}, \mathbf{w}^{n}(\mathbf{x})=\mathbf{w}\left(t_{n}, \mathbf{x}\right)$. We use a two-stage, second order singly diagonally implicit Runge-Kutta scheme (SDIRK) to discretize the system (5.1).

The properties of this method are detailed for example in [34]. In particular, it is only necessary to know how to solve a single implicit Euler step for the system (5.1) to apply this Runge-Kutta scheme, for which the semi-discretized variational formulation reads as follows. We write

$$
\mathbf{u}^{n+1}=\mathbf{u}^{n}+\Delta t \mathbf{k}_{u}, \quad \mathbf{v}^{n+1}=\mathbf{v}^{n}+\Delta t \mathbf{k}_{v} \quad \text { and } \quad \pi^{n+1}=\pi^{n}+\Delta t k_{\pi},
$$

where $\mathbf{k}_{u}, \mathbf{k}_{v} \in \mathbf{V}$ and $k_{\pi} \in L^{2}(\Omega)$ solve, for all $\mathbf{w} \in \mathbf{V}$ :

$$
\left\{\begin{aligned}
\int_{\Omega} \theta \rho \mathbf{k}_{v} \cdot \mathbf{w} & =-\int_{\Omega} \mathcal{A}^{h o m}\left(e\left(\mathbf{u}^{n}+\Delta t \mathbf{k}_{u}\right)\right): e(\mathbf{w}) \\
& +\int_{\Omega} k_{\pi} \mathcal{B}^{h o m}: e(\mathbf{w})+\int_{\Gamma_{N}} p_{N} \mathbf{w} \cdot \mathbf{n}, \\
\mathbf{k}_{u} & =\mathbf{v}^{n}+\Delta t \mathbf{k}_{v} \\
\left(\Delta t \mathrm{Id}+\tau_{h o m} \mathcal{R}\right) k_{\pi} & =-\pi^{n}-\mathcal{R}\left(\mathcal{B}^{h o m}: e\left(\mathbf{u}^{n}+\Delta t \mathbf{k}_{u}\right)\right) .
\end{aligned}\right.
$$

Space discretization To discretize correctly the non-local operator $\mathcal{R}$ using a uniform mesh size $h>0$, we begin by introducing a well-chosen truncation $\mathcal{O}_{h}$ of the infinite dyadic decomposition $\mathcal{O}=\left(\Omega_{n, k}\right)$ associated with the non-local operator $\mathcal{R}$ (see Definition 2.2). We make the assumption that the domain $\Omega$ and 


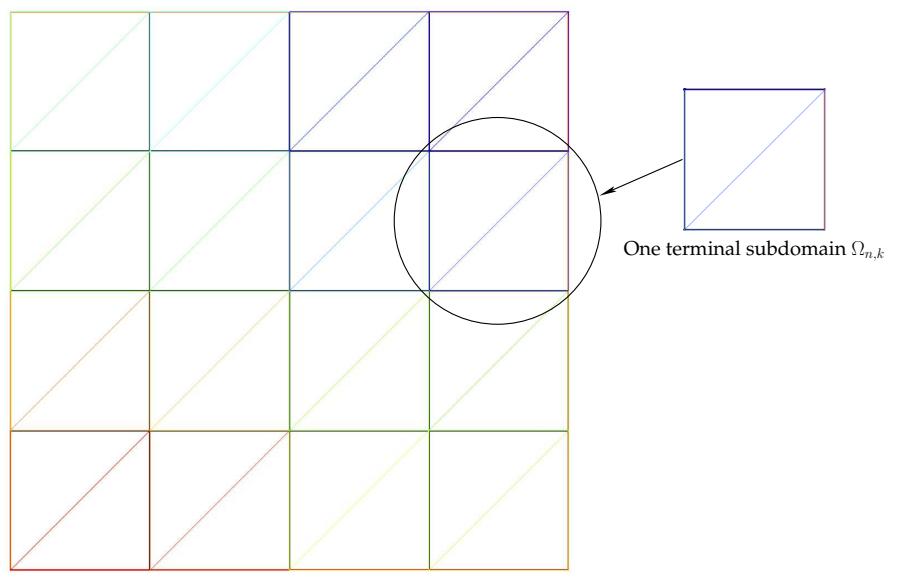

Figure 5: Mesh compatible with a 4-generation dyadic decomposition of the square.

all subdomains of the truncated, finite multi-scale decomposition $\mathcal{O}_{h}$ are polygonal and can be meshed uniformly with the mesh size $h$. We will then approximate the limit resistance operator $\mathcal{R}$ by a truncated operator $\mathcal{R}_{h}$. Note that $\mathcal{R}_{h}$ is obtained as the operator $\mathcal{R}_{\varepsilon}$ in Prop. 2.1, only truncating the dyadic decomposition at the mesh size $h$ instead of the alveolar length scale $\varepsilon$. The convergence of both these truncated operators can be obtained using the same methods (see also Remark 2.4).

As in Section 2, let us denote by $\mathcal{T}_{h}$ the finite dyadic tree associated with the dyadic decomposition $\mathcal{O}_{h}$ and $\mathcal{E}_{h}$ (resp. $\mathcal{I}_{h}$ ) the set of indices for the terminal (resp. interior) nodes of $\mathcal{T}_{h}$.

We introduce the following discretization spaces for the displacement and the pressure:

- the discrete finite elements space $\mathbf{V}_{h} \subset \mathbf{V}$, composed of $P 1$ elements,

- the discrete pressure space $M_{h}$ as the set of functions which are constant by cell on the subdomains $\Omega_{n, k}$ with $(n, k) \in \mathcal{E}_{h}$.

We now express the approximate solution as $\mathbf{u}_{h}^{n}, \mathbf{v}_{h}^{n} \in \mathbf{V}_{h}$ and $\pi_{h}^{n} \in M_{h}$. For any given function $\mathbf{w}$ in $\mathbf{V}_{h}$ or $\psi$ in $M_{h}$, we denote by $\hat{\mathbf{w}}$ or $\hat{\psi}$ the corresponding vector of unknowns.

\subsection{Numerical scheme}

We propose an algorithm that enables us to approximate efficiently the solution of problem (5.2). The matrix associated with $\mathcal{R}_{h}$ in $M_{h}$ is a symmetric definite positive matrix $A^{h} D_{h}$, where $A^{h}$ is the matrix associated with the resistances of the tree (see Prop. 1.1), and $D_{h}$ is the diagonal mass matrix of the triangulation $M_{h}$. 
Let us introduce the elastic FEM matrix $E_{\Delta t, h}$, which is associated with the following bilinear form on $\mathbf{V}_{h}$, and involves coefficients computed by solving cell problems:

$$
\left(\mathbf{u}_{h}, \mathbf{w}_{h}\right) \mapsto \int_{\Omega} \theta \rho \mathbf{u}_{h} \cdot \mathbf{w}_{h}+\Delta t^{2} \int_{\Omega} \mathcal{A}^{h o m} e\left(\mathbf{u}_{h}\right): e\left(\mathbf{w}_{h}\right)
$$

Let $B_{h}$ be the FEM matrix associated with the bilinear form on $\mathbf{V}_{h} \times M_{h}$ :

$$
\left(\mathbf{w}_{h}, k_{h}\right) \mapsto \int_{\Omega_{n, k}} k_{h} \mathcal{B}^{\text {hom }}: e\left(\mathbf{w}_{h}\right) .
$$

We derive from (5.2) a symmetric linear system with unknowns $\hat{\mathbf{k}}_{u}, \hat{\mathbf{k}}_{v}$ and $\hat{k}_{\pi}$, from which we eliminate $\hat{\mathbf{k}}_{v}$ using $(5.2 \mathrm{a})$ and then $\hat{k}_{\pi}$ by using a Schur complement approach. This results in the following linear system:

$$
\left(E_{\Delta t, h}+\Delta t B_{h}\left(\mathrm{Id}+\frac{\tau_{h o m}}{\Delta t} A^{h} D_{h}\right)^{-1} A^{h} B_{h}^{T}\right) \hat{\mathbf{k}}_{u}=\hat{\mathbf{F}}_{h}^{n}
$$

where the right-hand side $\hat{\mathbf{F}}_{h}^{n}$ is the vector obtained by collecting known terms corresponding to the previous timesteps as well as source terms.

The matrix appearing on the left-hand side of (5.3) is symmetric and positive definite. We use the conjugate gradient algorithm to solve the linear system, preconditioning by the matrix $E_{\Delta t, h}$ corresponding to the purely elastic problem. Note that in the incompressible case $\left(\tau_{h o m}=0\right)$, this method is the Uzawa algorithm applied to (5.2). Let us stress that the resistance matrix $A^{h}$ is not assembled: matrix-vector products involving $A^{h}$ or $\left(\operatorname{Id}+\frac{\tau_{h o m}}{\Delta t} A^{h} \mathcal{D}_{h}\right)^{-1}$ are computed as follows.

\section{Tree-based algorithms}

- First, we propose an efficient algorithm for computing the product $\mathbf{p}=A^{h} \mathbf{q}$, where $\mathbf{p}$ stands for a vector of pressures and $\mathbf{q}$ stands for a set of fluxes (see Section 1.3). Both $\mathbf{p}$ and $\mathbf{q}$ are indexed by $\mathcal{E}_{h}$. Let $N_{h}=\max \{n \mid(n, k) \in$ $\left.\mathcal{E}_{h}\right\}$ be the maximum length of the tree.

Given $\mathbf{q}=\left(q_{n, k}\right)_{(n, k) \in \mathcal{E}_{h}}$, compute $\mathbf{p}=A^{h} \mathbf{q}$ as follows:

(1) For $n=N_{h}-1, \ldots, 0$, evaluate and store the fluxes for all $k$ such that $(n, k) \in$ $\mathcal{I}_{h}$ thanks to the flux conservation law:

$$
q_{n, k}=q_{n+1,2 k}+q_{n+1,2 k+1} .
$$

(2) Set $p_{0}=0$ and $p_{0,0}=r_{0} q_{0,0}$.

(3) For $n=1, \ldots, N_{h}$, evaluate the pressures for all $k$ such that $(n, k) \in \mathcal{I}_{h} \cup \mathcal{E}_{h}$, thanks to the relation:

$$
p_{n, k}=p_{n-1, k / 2}+r_{n, k} q_{n, k} .
$$

(4) Obtain the vector $\mathbf{p}=\left(p_{n, k}\right)_{(n, k) \in \mathcal{E}_{h}}$.

Algorithm 5.1: Pressure algorithm 
- Next, we propose an efficient algorithm for computing $\mathbf{q}=$ $\left(\mathrm{Id}+\frac{\tau_{h o m}}{\Delta t} A^{h} D_{h}\right)^{-1} \mathbf{p}$ as follows. Recall that $D_{h}$ is a diagonal matrix whose entries are the volumes $\left|\Omega_{n, k}\right|$. Given $\omega>0$, we define the symmetric definite positive matrix $A_{\omega}^{h}=D_{h}^{-1}+\omega A^{h}$. Then, thanks to the Prop. 1.1, we observe that $A_{\omega}^{h}$ also represents the flux-to-pressure relation in the tree $\mathcal{T}_{h}$, with modified resistances $\left(r_{n, k}^{\omega}\right)$ defined by:

$$
r_{n, k}^{\omega}=\omega r_{n, k} \text { for }(n, k) \in \mathcal{I}_{h}, \quad r_{n, k}^{\omega}=\left|\Omega_{n, k}\right|^{-1}+\omega r_{n, k} \text { for }(n, k) \in \mathcal{E}_{h} .
$$

An idea to compute the set of fluxes q exiting this modified tree for a given set of pressures $\mathbf{p}$ is based on the concept of equivalent pressure and resistance of a subtree, similar to the concept of equivalent resistance in electric networks. Define recursively from the ends of the tree the equivalent resistance $R_{n, k}^{\omega}$ of the subtree stemming from the edge $X_{n-1, k / 2}-X_{n, k}$ as:

$$
R_{n, k}^{\omega}= \begin{cases}r_{n, k}^{\omega} & (n, k) \in \mathcal{E}_{h}, \\ r_{n, k}^{\omega}+\left(1 / R_{n+1,2 k}^{\omega}+1 / R_{n+1,2 k+1}^{\omega}\right)^{-1} & (n, k) \in \mathcal{I}_{h} .\end{cases}
$$

Given $\mathbf{p}=\left(p_{n, k}\right)_{(n, k) \in \mathcal{E}_{h}}$, compute $\mathbf{q}=D_{h}^{-1}\left(A_{\omega}^{h}\right)^{-1} \mathbf{p}$ as follows:

(1) Initialize the equivalent pressures at the ends of the tree as $P_{n, k}=p_{n, k}$, for $(n, k) \in \mathcal{E}_{h}$.

(2) For $n=N_{h}-1, \ldots, 0$, evaluate the equivalent pressures for all $k$ such that $(n, k) \in \mathcal{I}_{h}$, defined as:

$$
P_{n, k}=\frac{R_{n+1,2 k+1}^{\omega}}{R_{n+1,2 k}^{\omega}+R_{n+1,2 k+1}^{\omega}} P_{n+1,2 k}+\frac{R_{n+1,2 k}^{\omega}}{R_{n+1,2 k}^{\omega}+R_{n+1,2 k+1}^{\omega}} P_{n+1,2 k+1},
$$

and store the flux deviation defined as:

$$
d_{n, k}=\frac{P_{n+1,2 k}-P_{n+1,2 k+1}}{R_{n+1,2 k}^{\omega}+R_{n+1,2 k+1}^{\omega}} .
$$

(3) Set $\phi_{0,0}=P_{0,0} / R_{0,0}^{\omega}$.

(4) For $n=0, \ldots, N_{h}-1$ and for all $k$ such that $(n, k) \in \mathcal{I}_{h}$, evaluate the fluxes $\phi_{n+1,2 k}$ and $\phi_{n+1,2 k+1}$ thanks to the relation:

$$
\begin{aligned}
& \phi_{n+1,2 k}=\frac{R_{n+1,2 k+1}^{\omega}}{R_{n+1,2 k}^{\omega}+R_{n+1,2 k+1}^{\omega}} \phi_{n, k}+d_{n, k}, \\
& \phi_{n+1,2 k}=\frac{R_{n+1,2 k}^{\omega}}{R_{n+1,2 k}^{\omega}+R_{n+1,2 k+1}^{\omega}} \phi_{n, k}-d_{n, k} .
\end{aligned}
$$

(5) Obtain the vector $\mathbf{q}=\left(\left|\Omega_{n, k}^{h}\right|^{-1} \phi_{n, k}\right)_{(n, k) \in \mathcal{E}_{h}}$.

Algorithm 5.2: Flux algorithm 
Clearly, the cost of both algorithms and the memory requirements are linear with respect to the number $N$ of degrees of freedom of $M_{h}$, since the tree $\mathcal{T}_{h}$ contains at most $2 N$ vertices.

\subsection{Numerical results}

We present in this section a few numerical results. We used the finite element software FreeFem $++[21]$ to perform all computations.

Choice of parameters. In order to test our model, the domain $\Omega$ is a twodimensional square domain with a side measuring $40 \mathrm{~cm}$, and the mesh size $h$ is chosen so that the truncated dyadic decomposition $\mathcal{O}_{h}$ can be associated to a geometric resistive tree with 12 generations as described in Section 2.2.1. The right side of the square domain is chosen as the Dirichlet boundary $\Gamma_{D}$. The computation of the homogenized elastic coefficients was performed on an hexagonal periodic cell for an incompressible material, as presented on Figure 6. To obtain the entries of the elastic tensor $\mathcal{A}^{\text {hom }}$ we solve numerically the elliptic cell problems (4.18). We refer the reader to [3] for a detailed numerical study of the homogenized coefficients as a function of the Lamé parameters. We remind that the parameter $\alpha$ controls the geometric increase of resistances in the tree with the generation number, see Definition 2.3.

Remark 5.2. In this numerical section, we do not restrict ourselves to the particular geometric setting used to prove the convergence of $\mathcal{R}_{\varepsilon}$ in Section 2. This allows us for example to use a hexagonal periodic cell ensuring an isotropic homogenized medium, which is not the case for a square cell. This choice is also a better fit to the geometry of the alveoli. Rigorous proofs of convergence in this case will be detailed in a forthcoming paper.

Remark 5.3. We assume a constant $5 \mathrm{~cm}$ height in the computations used to convert the variations in area to more usual volume units for the figures.
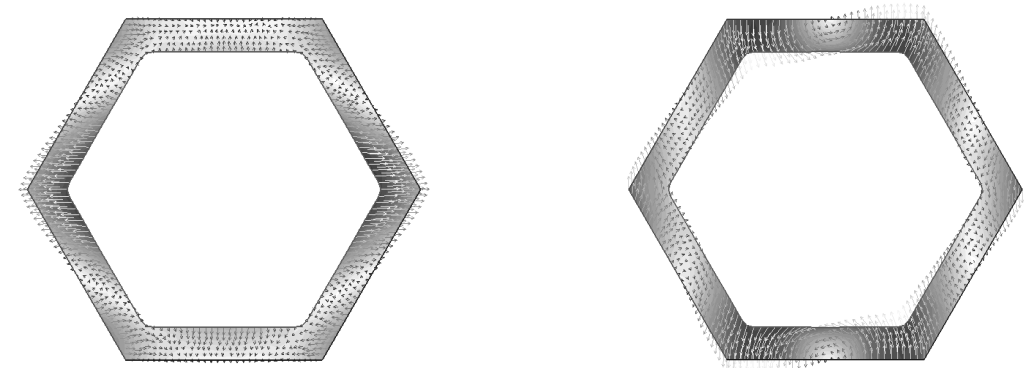

Figure 6: Cell geometry and periodic correctors, $\chi^{1,1}$ (left) and $\chi^{1,2}$ (right) 


\begin{tabular}{ccccccc}
\hline$\mu$ & $\lambda_{\text {hom }}$ & $\mu_{\text {hom }}$ & $\tau_{\text {hom }}$ & $\theta$ & $\alpha$ & $r_{0}$ \\
\hline $1 e 5 \mathrm{~Pa}$ & $2820 \mathrm{~Pa}$ & $637 \mathrm{~Pa}$ & $0 \mathrm{~Pa}^{-1}$ & 0.15 & 1.63 & $3200 \mathrm{~Pa} \cdot \mathrm{s} / \mathrm{m}^{2}$ \\
\hline
\end{tabular}

Table 1: Parameters for the numerical computations (incompressible case)

Academic test cases. We present here a few snapshots of the propagation of a pressure wave for different values of the parameters $\tau_{\text {hom }}$ and $\alpha$ in Figure 7 in order to better understand the homogenized model (5.1). These computations are based on some numerical values of the parameters presented in Table 1 as a reference, corresponding to the incompressible case. Note that incompressibility of the alveolar wall material is a reasonable assumption in the case of the human lung since it is composed mainly of water. These test cases are obtained as follows: starting at $t=0$, a sine pressure wave with frequency $50 \mathrm{~Hz}$ is applied to the left side of the domain filled with the homogenized material, which is initially at rest. Snapshots of the fluid pressure field at $t=0.045 \mathrm{~s}$ are presented in Figure 7 .

- We observe that when $\tau_{\text {hom }}$ increases from 0 to $10^{-3}$ with $\alpha=1.6$ fixed, the wavelength decreases and the amplitude of the wave diminishes. Indeed, when $\tau_{\text {hom }}=0$ (incompressible case) the pressure wave propagates at infinite velocity and there are no nonlocal effects in time, see Remark 4.2. When $\tau_{\text {hom }}>0$, pressure waves can propagate inside the homogenized material at a finite velocity, which depends inversely on $\tau_{\text {hom }}$.

- When $\alpha$ decreases from 1.6 to 1 with $\tau_{\text {hom }}=0$ fixed, we observe that the distribution of pressure inside the material is less regular and that there appear important nonlocal, long-range effects in space. Indeed, when $\alpha$ is small the air can easily flow through the airways, so the alveolar pressure in distant parts of the material tends to balance.

Simulation of spirometry experiments We have performed some numerical experiments using the pressure profile presented in Figure 8 which we discuss next. The corresponding medical test would run as follows.

- The "patient" breathes normally for a few seconds.

- After a short while, the "patient" takes a deep inspiration, and then tries to exhale as fast as possible.

The phase portrait (instantaneous flux vs. inhaled volume) obtained in these conditions is the curve obtained by spirometry, and provides pneumologists with information useful to diagnose some respiratory pathologies presented by the patient.

Reference case The curves in Figure 8 agree very closely with the results obtained with a simple linear one-compartment model for the lung, see [5]. This is a consequence of the perfect symmetry of the domain and tree used in the computations, 

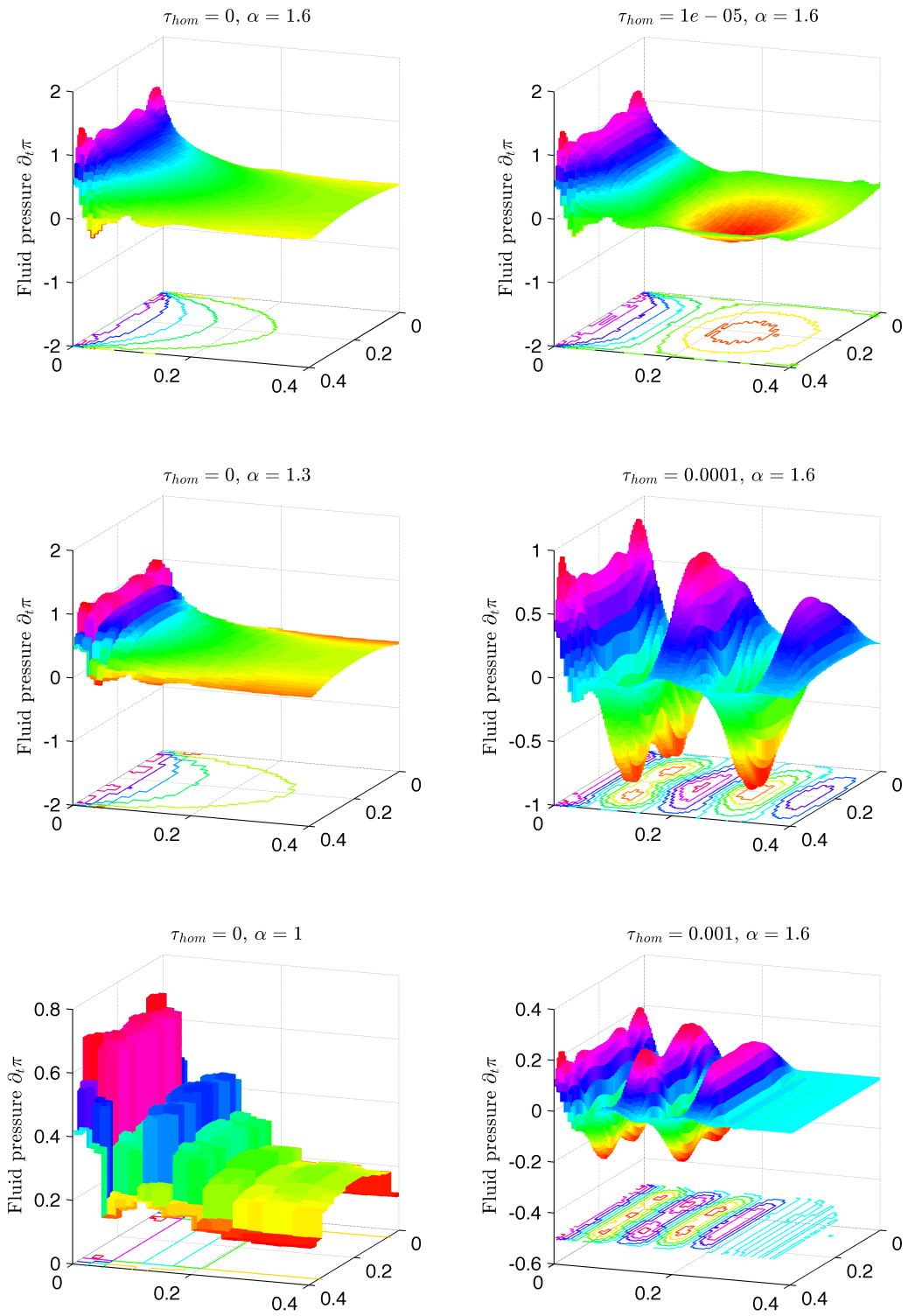

Figure 7: Snapshots of the propagation of a pressure wave in the homogenized medium for various sets of parameters, taken at the same time $t=0.045 \mathrm{~s}$. 
modeling a perfectly homogeneous lung.

Remark 5.4. We are limited by the linearity of the mechanical model, in particular in this scenario of forceful expiration. For example, it would be necessary to solve the full Navier-Stokes equations in the first generations of the bronchial tree to capture the nonlinearities due to inertial effects at such high Reynolds numbers. In particular, the real pleural pressure during forceful expiration is ten times higher that the values used here, see Figure 8. Nevertheless, some interesting aspects can already be seen from the simulations obtained here. We refer e.g. to [4] for simulations obtained by computing the airflow in this complex geometry, but modeling the elastic behavior of the parenchyma by a single spring.

Parametric study The model allows us to investigate the influence of local perturbations on the phase diagram, and in particular the effect of random local changes in the parameters. As an example, we show in Figure 9 the different effect of random perturbations on the airway diameters in the reference case compared to a case where the diameters are also systematically reduced by $40 \%$ in one half only of the domain. The first case can be associated to a normal lung while the second represents a diseased lung with bronchitis or some localized asthma.

- Even without random perturbations, maximal flow and change in volume are reduced in the asthmatic case. We also observe that the asymmetry induces a concave profile because each side empties or fills at a different rate.

- In addition, there is a marked difference in the effect of the random diameter perturbations in each case. In the normal case, the maximal expiratory flow is reduced but the maximal and minimal total lung volume are not effected. In the diseased case however, the random perturbations induce a marked reduction in both flow and lung volume: some parts of the lung become closed to the ventilation process.

Such examples show that this model could be useful in reproducing some spatially localized pathological features (emphysema, bronchitis, tumors...)

\section{Bibliography}

1. G. Allaire. Homogenization and two-scale convergence. SIAM J. Math. Anal., 23(6):1482-1518, 1992.

2. G. Allaire and C. Conca. Bloch-wave homogenization for a spectral problem in fluid-solid structures. Arch. Rational Mech. Anal., 135:197-257, 1996.

3. L. Baffico, C. Grandmont, Y. Maday, and A. Osses. Homogenization of elastic media with gaseous inclusions. Multiscale Model. Simul., 7(1):432-465, 2008.

4. L. Baffico, C. Grandmont, and B. Maury. Multiscale modeling of the respiratory tract. Math. Models Methods Appl. Sci., 20(1):59-93, 2010.

5. J. H. T. Bates. Lung Mechanics, An Inverse Modeling Approach. Cambridge University Press, 2009. 
6. P. Cazeaux. Quelques modèles mathématiques homogénéisés appliqués à la modélisation du parenchyme pulmonaire. PhD thesis, Université Pierre et Marie Curie, December 2012.

7. J. R. Cebral and R. M. Summers. Tracheal and central bronchial aerodynamics using virtual bronchoscopy and computational fluid dynamics. IEEE Trans. Med. Imaging, 23(8):1021-1033, 2004.

8. P. G. Ciarlet. Mathematical elasticity. Vol. I. Three-dimensional elasticity, volume 20 of Studies in Mathematics and its Applications. North-Holland Publishing Co., Amsterdam, 1988.

9. D. Cioranescu and P. Donato. Exact internal controllability in perforated domains. $J$. Math. Pures Appl. (9), 68(2):185-213, 1989.

10. C. Conca. On the application of the homogenization theory to a class of problems arising in fluid mechanics. J. Math. Pures Appl. (9), 64(1):31-75, 1985.

11. G. Duvaut and J.-L. Lions. Les inéquations en mécanique et en physique. Dunod, Paris, 1972. Travaux et Recherches Mathématiques, No. 21.

12. M. Fabrizio and A. Morro. Mathematical problems in linear viscoelasticity, volume 12 of SIAM Studies in Applied Mathematics. Society for Industrial and Applied Mathematics (SIAM), Philadelphia, PA, 1992.

13. M. Fang, R. P. Gilbert, A. Panchenko, and A. Vasilic. Homogenizing the timeharmonic acoustics of bone: The monophasic case. Mathematical and computer modelling, 46(3-4):331-340, 2007.

14. J. L. Ferrín and A. Mikelić. Homogenizing the acoustic properties of a porous matrix containing an incompressible inviscid fluid. Math. Methods Appl. Sci., 26(10):831-859, 2003.

15. C. Fetita, S. Mancini, D. Perchet, F. Prêteux, M. Thiriet, and L. Vial. An image-based computational model of oscillatory flow in the proximal part of tracheobronchial trees. Computer Methods in Biomechanics and Biomedical Engineering, 8(4):279-293, 2005.

16. R. P. Gilbert and A. Mikelić. Homogenizing the acoustic properties of the seabed. I. Nonlinear Anal., 40(1-8, Ser. A: Theory, Methods):185-212, 2000.

17. V. Girault and P.-A. Raviart. Finite element methods for Navier-Stokes equations, volume 5 of Springer Series in Computational Mathematics. Springer, Berlin, 1986. Theory and algorithms.

18. C. Grandmont, B. Maury, and N. Meunier. A viscoelastic model with non-local damping, application to the human lungs. Mathematical Modelling and Numerical Analysis, 40(1):201-224, 2006.

19. Q. Grimal, A. Watzky, and S. Naili. A one-dimensional model for the propagation of transient pressure waves through the lung. Journal of Biomechanics, 35(8):1081-1089, 2002 .

20. A. Hanyga. Viscous dissipation and completely monotonic relaxation moduli. Rheologica Acta, 44:614-621, 2005.

21. F. Hecht. FreeFem ++ manual, 2012.

22. Y. Lanir. Constitutive equations for the lung tissue. J Biomech Eng, 105(4):374-380, Nov 1983.

23. S. Ley, D. Mayer, B. S. Brook, E. J. Van Beek, C. P. Heussel, D. Rink, R. Hose, K. Markstaller, and H. U. Kauczor. Radiological imaging as the basis for a simulation software of ventilation in the tracheo-bronchial tree. Eur. Radio., 12(9):2218-2228, 2002.

24. J.-L. Lions. Quelques méthodes de résolution des problèmes aux limites non linéaires. Dunod, 1969.

25. J.-L. Lions and E. Magenes. Problèmes aux limites non homogènes et applications. 
Vol. 1. Travaux et Recherches Mathématiques, No. 17. Dunod, Paris, 1968.

26. B. B. Mandelbrot. The fractal geometry of nature. W. H. Freeman and Co., San Francisco, Calif., 1982.

27. S. Martin, T. Similowski, C. Straus, and B. Maury. Impact of respiratory mechanics model parameters on gas exchange efficiency. In Mathematical and numerical modelling of the human lung, volume 23 of ESAIM Proc., pages 30-47. EDP Sci., Les Ulis, 2008.

28. B. Mauroy, M. Filoche, J. Andrade, and B. Sapoval. Interplay between geometry and flow distribution in an airway tree. Phys. Rev. Lett., 90(14), Apr 2003.

29. B. Mauroy, M. Filoche, E. Weibel, and B. Sapoval. An optimal bronchial tree may be dangerous. Nature, pages 633-636, Jan 2004.

30. R. E. Miller. Homogenization of time-dependent systems with Kelvin-Voigt damping by two-scale convergence. Discrete Contin. Dynam. Systems, 1(4):485-502, 1995.

31. G. Nguetseng. A general convergence result for a functional related to the theory of homogenization. SIAM J. Math. Anal., 20(3):608-623, 1989.

32. G. Nguetseng. Asymptotic analysis for a stiff variational problem arising in mechanics. SIAM J. Math. Anal., 21(6):1394-1414, 1990.

33. M. R. Owen and M. A. Lewis. The mechanics of lung tissue under high-frequency ventilation. SIAM J. Appl. Math., 61(5):pp. 1731-1761, 2001.

34. B. Owren and H. H. Simonsen. Alternative integration methods for problems in structural dynamics. Comput. Methods Appl. Mech. Engrg., 122(1-2):1-10, 1995.

35. E. Sanchez-Palencia. Vibration of mixtures of solids and fluids. In Non-Homogeneous Media and Vibration Theory, volume 127 of Lecture Notes in Physics, pages 158-190. Springer, 1980.

36. M. Siklosi, O. E. Jensen, R. H. Tew, and A. Logg. Multiscale modeling of the acoustic properties of lung parenchyma. ESAIM: Proc., 23:78-97, 2008.

37. M. H. Tawhai, A. J. Pullan, and P. J. Hunter. Generation of an anatomically based three-dimensional model of the conducting airways. Annals of Biomedical Engineering, 28:793-802, 2000.

38. C. Vannier. Modélisation mathématique du poumon humain. PhD thesis, Université Paris-Sud (Orsay), Jul 2009.

39. C. Vannier, D. Salort, and B. Maury. Trace theorems for trees and application to the human lungs. Networks and Heterogeneous Media, 4(3):469-500, Jul 2009.

40. E. R. Weibel. Morphometry of the human lung. Springer, page 151, Jan 1963.

41. E. R. Weibel. The pathway for oxygen. Harvard Press, Cambridge, Mass., Jan 1984. 

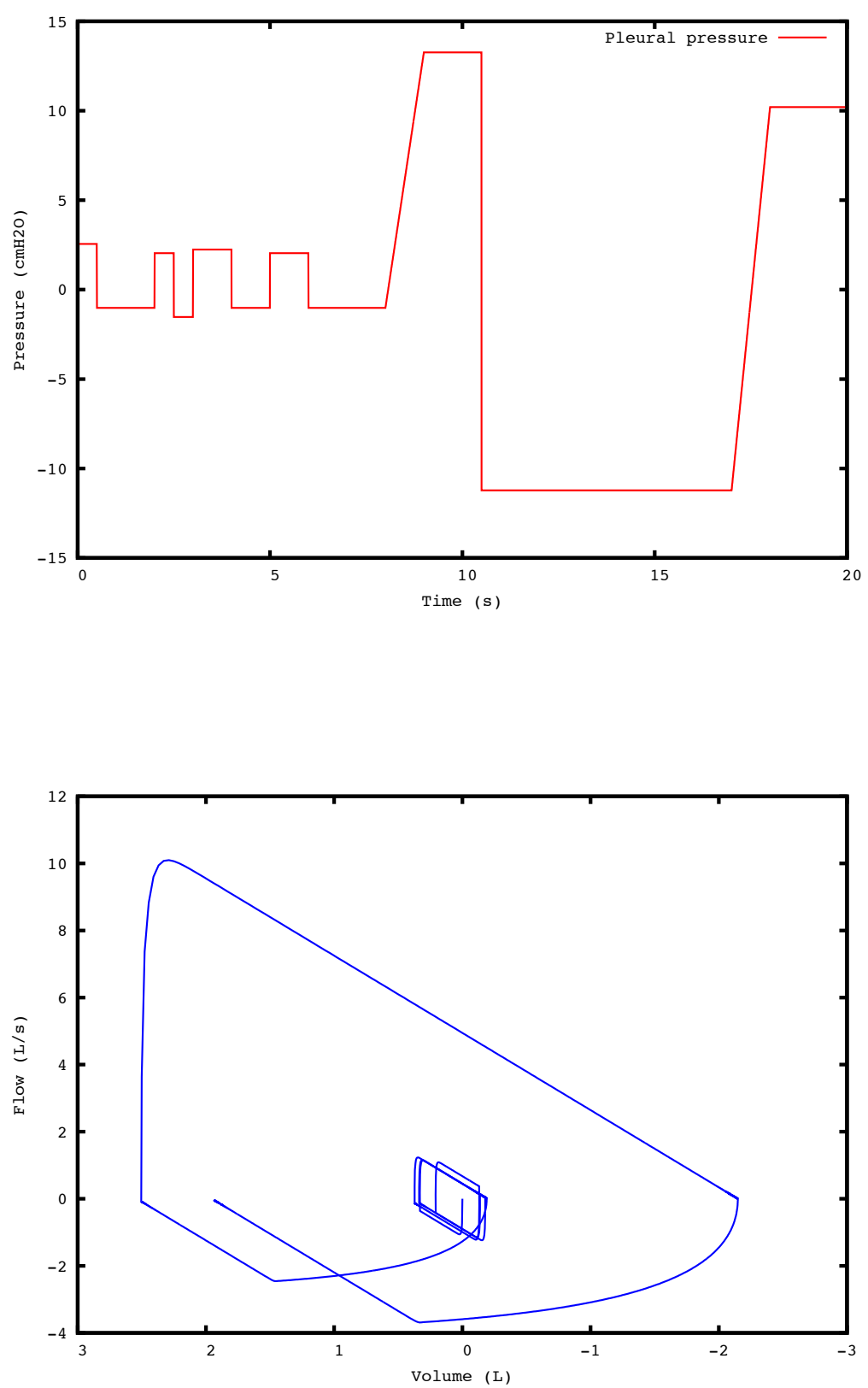

Figure 8: Pressure vs time (up) and phase portrait (down) 


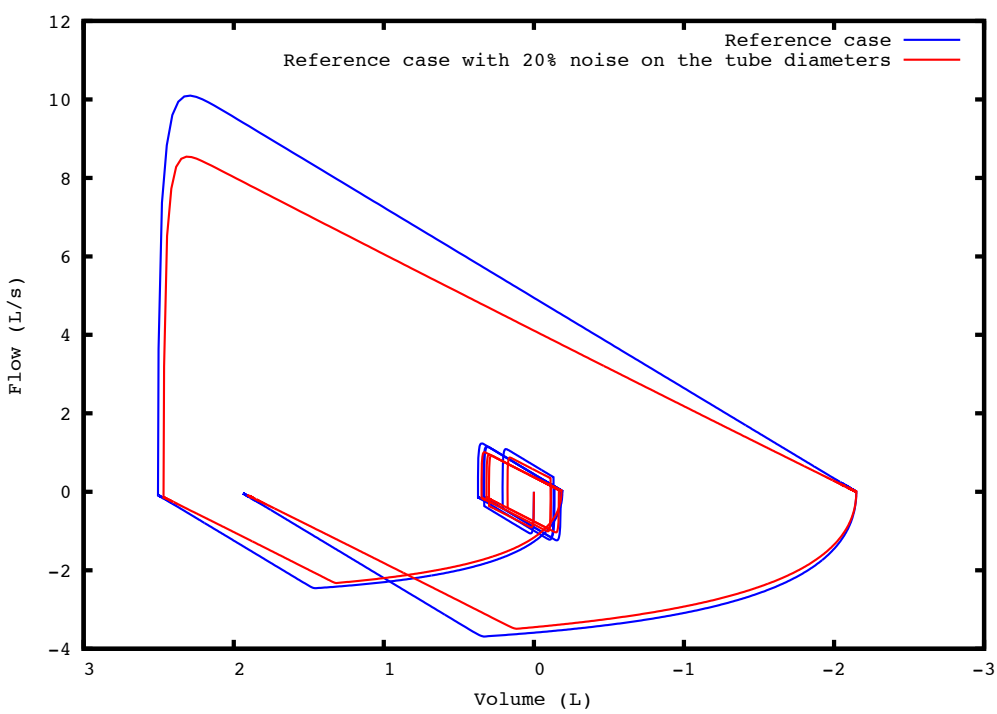

(a) Reference case: symmetric Poiseuille resistances (geometric case)

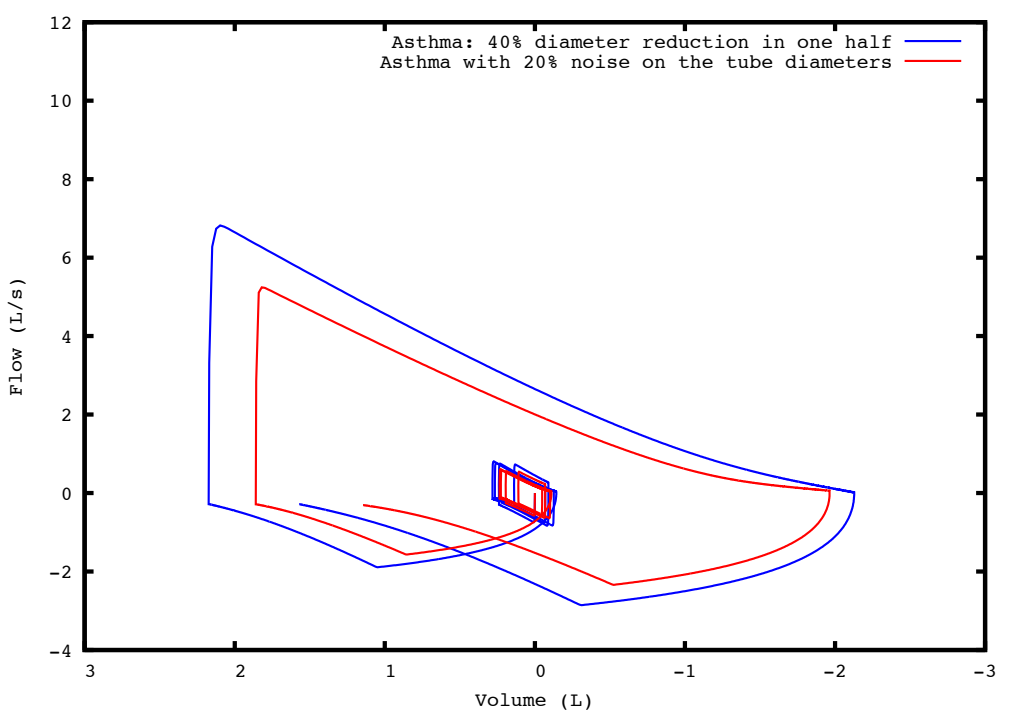

(b) Asthmatic case: systematic increase of the resistances in one half of the domain

Figure 9: Phase diagrams in the reference case (a) and asthmatic case (b) 